\title{
LA SEMICONTINUITÀ NEL CALCOLO DELLE VARIAZIONI.
}

\author{
Memoria di Leonida Tonelli (Parma).
}

Adunanza del 23 novembre 1919.

INTRODUZIONE.

In alcuni lavori, pubblicati in questi stessi Rendiconti ${ }^{\mathrm{I}}$ ), partendo dal concetto che il successo dei metodi proposti da Hilbert e da altri per la dimostrazione « a priori " dell'esistenza del minimo nel problema di Dirichlet e suoi derivati, si deve in gran parte alla semicontinuità degli integrali che in tali problemi entrano in gioco, stabilii questa semicontinuità per tutti gli integrali curvilinei di una vasta classe di problemi di Calcolo della Variazioni, dimostrando poi, per mezzo di essa, l'esistenza del minimo per gli integrali regolari soddisfacenti ad opportune condizioni complementari.

La dimostrazione della semicontinuita, quale trovasi nei citati lavori, si presenta alquanto laboriosa, perchè basata sul confronto di varie definizioni, proposte da diversi Autori, per l'integrale preso su una curva continua di cui si supponga soltanto la rettificabilita. Inoltre, essa gioca tutta sulla gia supposta esistenza della soluzione dei relativi problemi di minimo o massimo in piccolo, vale a dire in campi sufficientemente ristretti. E questo diventa un inconveniente di notevole importanza quando si voglia, come intendo fare io, trattare a nuovo, i problemi di Calcolo delle Variazioni con un metodo diretto fondato sulla semicontinuità degli integrali che si considerano.

Questi inconvenienti, e più specialmente il primo qui menzionato, furono rilevati anche dal Goursat ${ }^{2}$ ), il quale, ad evitarli, propose di porre la semicontiuuita a base della definizione stessa di integrale su una curva rettificabile, e ciò in armónia con quanto aveva gì̀ fatto il Lebesgue definendo la lunghezza di una curva come il più

I) L. Tonelli, Sui massimi e minimi assoluti del Calcolo delle Variazioni [Rendiconti del Circolo Matematico di Palermo, t. XXXII ( $2^{\circ}$ semestre I9II), pp. 297-337]; Sul caso regolare nel Calcolo delle Variazioui [Ibidem; t. XXXV (I ${ }^{\circ}$ semestre 191 3 ), pp. 49-73].

${ }^{2}$ ) E. Goursat, Sur quelques fonctions de lignes semi-continues [Bulletin de la Société Mathématique de France, t. XLIII, fasc. II (I9I5), pp. II8-130]. 
piccolo limite delle lunghezze delle poligonali che tendono uniformemente alla curva medesima.

La proposta del Goursat, più che a vincere le difficoltà della questione, tende a scansarle; in fatto poi non fa che rimandarne la soluzione ad un momento ulteriore. Ed invero, se, con la nuova definizione, si puó facilmente giungere a stabilire, sotto certe condizioni, l'esistenza dell'estremo, gravi difficoltà per essa si presentano quando si debbano stabilire le proprietà analitiche dell'estremante. Di più, esistendo, da un lato, l'integrale della funzione che si considera su una data curva rettificabile - l'integrale essendo preso secondo la definizione fissata dal Lebesgue per le funzioni misurabili e ormai universalmente accettata nel campo dell'Analisi - e non potendo, dall'altro, dar sempre all'integrale stesso un significato geometrico ben chiaro, che legittimi a priori l'ipotesi della semicontinuita, si viene, con la definizione proposta, in alcuni casi, a trovarsi nella necessità di accettare la definizione stessa, giustificandola soltanto con il suo accordo con quella abituale, quando si tratti di curve ordinarie ${ }^{3}$ ); in altri, a creare un dualismo apparente, relativo a due problemi - uno analitico (riguardante lintegrale del LeBESGUE) ed uno geometrico - perfettamente equivalenti; e sempre poi a rifiutare, señza notevoli vantaggi, quella definizione di integrale che si presenta come la più legittima. Si deve inoltre aggiungere, a titolo decisivo, che, contemporaneamente alla Memoria citata del Goursa's, se ne pubblicava un'altra ${ }^{4}$ ) nella quale, pur conservando per l'integrale la definizione analitica del LeBesgue, tutti gli inconvenienti sopra esposti venivano ad esser superati. Ed è opportuno osservare che la dimostrazione della semicontinuità per le curve rettificabili contenuta in questo nuovo lavoro, è, per metodo e per semplicità, del tutto analoga a quella che il Goursat diede per le curve ordinarie.

Nella Memoria sopra indicata, considerai le curve date in forma non parametrica e sottoposi la funzione integranda $f\left(x, y, y^{\prime}\right)$, oltre alla condizione $f_{y^{\prime} y^{\prime}} \geqslant 0$, a una condizione relativa al suo ordine di infinito per $y^{\prime} \rightarrow \infty$. Qui - sempre limitandomi agli integrali curvilinei dipendenti soltanto da derivate del primo ordine - mi propongo di riprendere in esame tutta la questione della semicontinuità, per le curve date in forma parametrica e per quelle in forma non parametrica; di studiare non soltanto

3) Composte cioè di un numero finito di archi di curva aventi tangente variabila in modo continuo.

4) L. Tonelin, Sur une methode directe du Calcul des Variations [Rendiconti del Circolo Matematico di Palermo, t. XXXIX ( ${ }^{\circ}$ semestre I9Is), Pp. 233-264]. 
le condizioni sufficienti ma anche quelle necessarie; e di procedere sempre con il solo ausilio della definizione di integrale di LEBESGUE e senza ricorrere per nulla a risultati del Calcolo delle Variazioni.

Per gli integrali

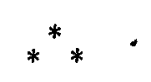

$$
\int_{c} F\left(x, y, x^{\prime}, y^{\prime}\right) d s
$$

relativi a curve date in forma parametrica, si vedrà che la condizione

$$
F_{1} \geqslant 0 \text {, }
$$

dove $F_{\mathrm{s}}$ è l'invariante di Weierstrass definito da

$$
F_{1} \equiv \frac{F_{x^{\prime} x^{\prime}}}{y^{\prime 2}} \equiv-\frac{F_{x^{\prime} y^{\prime}}}{x^{\prime} y^{\prime}} \equiv \frac{F_{y^{\prime} y^{\prime}}}{x^{\prime 2}}
$$

è sufficiente per la semicontinuitd inferiore $\left(F_{1} \leqslant 0\right.$, per quella superiore) se in nessun punto del campo che si considera la $F_{1}=0$ risultá soddisfatta per tutte le possibili coppie $\left(x^{\prime} y^{\prime}\right)$. La presenza di questi punti eccezionali può veramente in alcuni casi escludere la semicontinuità. La condizione $F_{\mathrm{r}} \supseteq 0$, verificata senz'altra restrizione, porta solo alla semicontinuità inferiore rispetto alla classe delle curve le cui lunghezze restano inferiori ad un numero fisso, del resto qualunque. Se esistono punti eccezionali, per assicurare la completa semicontinuitd (inferiore), occorre aggiungere una condizione complementare, come ad esempio quella espressa dalla disuguaglianza $F \supseteq 0$, valida in tutto un intorno dei punti eccezionali medesimi. E saranno date anche altre forme notevoli di condizioni complementari.

Quando poi tutti i punti del campo siano punti eccezionali, nel qual caso la funzione $F$ prende la forma $P(x y) x^{\prime}+Q(x y) y^{\prime}$, si giungerd alla condizione necessaria e sufficiente per la semicontinuità (condizione che coincide quil con quella della continuitd), espressa dall'uguaglianza

$$
\frac{\partial P}{\partial y}=\frac{\partial Q}{\partial x},
$$

verificata in tutto il campo considerato (ciò almeno per $i$ campi che ordinariamente si presentano).

Nel caso generale, si troverd che sono condizioni necessarie affinchè, su ogni curva completamente interna al campo considerato, si abbia la semicontinuitd inferiore (superiore), $\left.\mathrm{I}^{\circ}\right)$ che sia sempre, nell'interno del campo, $\left.F_{\mathrm{I}} \geqslant 0\left(F_{1} \leqslant 0\right) ; 2^{\circ}\right)$ che, in ogni punto interno al campo in cui sia $F_{\mathrm{r}} \equiv \mathrm{o}$, per tutte le possibili coppie $\left(x^{\prime} y^{\prime}\right)$, risulti $F_{x^{\prime} y} \equiv F_{y^{\prime} x}$.

Rend. Circ. Matem. Palermo, t. XLIV (1920). - Stampato il 14 settembre 1920. 
Si studierà anche la semicontinuità sopra una data curva (non più su tutte le curve del campo) e, fatto notevole, si provera che la cosidetta quarta condizione sufficiente per l'estremo, del Calcolo delle Variazioni (che è la condizione aggiunta da WeIERSTRASs), assicura la semicontinuità su quella qualsiasi curva su cui sia verificata; si troverd poi che, come condizioni necessarie per la semicontinuità, devono esser soddisfatte quasi ovunque, sulla curva data, la condizione di LEgENDre e la cosidetta quarta condizione necessaria per l'estremo, pure del Calcolo delle Variazioni.

Per gli integrali

$$
\int_{C} f\left(x, y, y^{\prime}\right) d x,
$$

relativi a curve, date in forma non parametrica, si dimostrerà che condizione necessaria e sufficiente per la semicontinuità inferiore (superiore), su ogni curva del campo che si considera, è che sia sempre in esso

$$
f_{y^{\prime} y^{\prime}} \supseteq 0, \quad\left(f_{y^{\prime} y^{\prime}} \leq 0\right)
$$

Per la semicontinuità su una data curva, si troverd anche quì che è sufficiente la quarta condizione sufficiente per l'estremo, del Calcolo delle Variazioni, e che sono necessarie la condizione di LEGENDRE e la quarta condizione necessaria per l'estremo, pure del Calcolo delle Variazioni, le quali condizioni devono esser verificate quasi ovunque sulla curva considerata.

I risultati qui ottenuti pongono in piena luce il significato della condizione di LEGENDRE e della quarta condizione (condizione di WeIERSTRAss) del Calcolo delle Variazioni. Queste condizioni risultano equivalere alla semicontinuita, sulla curva considerata, dell'integrale di cui si tratta, e resta cosi evidente come esse siano indispensabili per l'esistenza dell'estremo; d'altra parte, poichè, per poter asserire che una data curva, su cui tali condizioni siano verificate, dà un estremo, basta mostrare che su essa è nulla la variazione prima dell'integrale in quistione e sempre di un segno (non nulla) la variazione seconda, ne risulta una perfetta corrispondenza fra il Calcolo delle Variazioni e la teoria dei massimi e minimi delle ordinarie funzioni continue, per le quali si ha sicuramente un estremo là dove è nulla la derivata prima e diversa da zero la derivata seconda. 


\section{CAPITOLO I. \\ GLI INTEGRALI PER LE CURVE IN FORMA PARAMETRICA.}

\section{$\int \mathrm{I}$.}

\section{Definizioni.}

I. Sia $A$ un campo (insieme di punti) del piano $(x y)$ tale che i suoi punti appartenenti ad un qualsiasi cerchio dello stesso piano costituiscano sempre un insieme chiuso. Un punto $P$ si dird interno ad $A$ se esisterd un cerchio, avente il centro in tal punto, tutto costituito di punti di $A$.

Si chiamerd frontiera di $A$ l'insieme dei punti di $A$ non interni al campo stesso.

2. Sia $F\left(x, y, x^{\prime}, y^{\prime}\right)$ una funzione:

a) definita e continua, insieme con le sue derivate parziali dei primi due ordini rispetto a $x^{\prime}$ e $y^{\prime}$, per tutti i punti $(x y)$ del campo $A$ e per tutte le coppie $\left(x^{\prime} y^{\prime}\right)$ di numeri non ambedue nulli;

b) positivamente omogenea, di grado I, rispetto alle $x^{\prime}, y^{\prime}$, vale a dire soddisfacente alla uguaglianza

$$
F\left(x, y, k x^{\prime}, k y^{\prime}\right)=k F\left(x, y, x^{\prime}, y^{\prime}\right),
$$

valida per ogni $k>0$.

c) avente continue, in tutti i punti del campo $A$ e per tutte le coppie $\left(x^{\prime} y^{\prime}\right)$ di numeri non ambedue nulli, le derivate parziali prime rispetto alla $x$ ed alla $y$ della $F_{x^{\prime}}$, $F_{y}$ e della funzione $F_{1}$ definita nell'introduzione ${ }^{5}$ ).

3. Si consideri la curva continua e rettificabile $C$, appartenente tutta al campo $A$ e definita dalle equazioni

$$
x=\varphi(t), y=\psi(t)
$$

Queste equazioni stabiliscono anche il verso della curva. Per noi ogni curva avrà un verso ed uno solo; e pertanto i segmenti rettilinei $P Q$ e $Q P$, ad esempio, saranno per noi due curve distinte.

Assumendo come parametro la lunghezza $s$ dell'arco che dal punto corrispondente

5) La continuità la si intenderà sempre rispetto al complesso delle quattro variabili $x, y, x^{\prime}, y^{\prime}$. Si vedrà che la condizione $c$ ) non interviene se non dal $\mathrm{n}^{\circ} \mathrm{I} 6$ in poi. 
a $t_{0}$ va a quello relativo a $t$, le equazioni della $C$ prendono la forma

$$
x=x(s), \quad y=y(s), \quad(0 \leq s \leq L),
$$

dove $L$ indica la lunghezza di tutta la curva.

Nel seguito saranno considerate sempre curve continue e rettificabili del campo $A$.

4. Considerata una curva $C$ e scelto un numero positivo $\rho$, si dirà che un'altra curva $C^{\prime}$ appartiene ordinatamente all'intorno $(p)$ della $C$ se sarà possibile di porre fra i punti delle due curve una corrispondenza biunivoca, ordinata ${ }^{6}$ ) e continua, tale che la distanza fra due punti corrispondenti qualsiasi risulti sempre minore del numero $\hat{p}$.

5. Formiamo l'integrale (di Lebesgue) della funzione $F$, esteso alla curva $C$, vale 2 dire,

$$
I_{C}=\int_{C} F\left(x(s), y(s), x^{\prime}(s), y^{\prime}(s)\right) d s,
$$

che scriveremo semplicemente

$$
\int_{C} F d s
$$

Questo integrale, fissata che sia la forma della funzione $F$, dipende unicamente dalla curva $C$ su cui l'integrazione è eseguita ed ha un valore determinato e finito per ogni curva: esso è perciò una funzione della curva $C$.

E chiaro che, se la curva è chiusa, gli archi si possono contare a partire da un'origine arbitrariamente scelta sulla curva stessa senza alterare il valore di $I_{C}$.

6. Diremo che l'integrale $I_{C}$ è regolare se l'invariante di Weinrstrass $F_{x}$ è sempre di un segno, senza mai aunullarsi, per tutti i punti $(x y)$ del campo $A$ e per tutte le coppie $\left(x^{\prime}, y^{\prime}\right)$ di numeri non ambedue nulli; diremo, invece, che $I_{C}$ è un integrale quasi regolare se, per $\mathrm{i}$ valori indicati di $x, y, x^{\prime}, y^{\prime}, F_{\mathrm{x}}$ è sempre di un segno, non escluso che possa anche annullarsi.

Diremo, infine, che l'integrale $I_{C}$ è definito ${ }^{7}$ ) se la funzione $F$ conserva sempre uno stesso segno, senza mai annullarsi, per tutti i valori di $x, y, x^{\prime}, y^{\prime}$, gia detti, e, più precisamente, che è definito positivo se è sempre $F>0$, definito negativo se invece è sempre $F<0$.

7. $\mathrm{Si}$ dirà che l'integrale $I_{C}$ è una funzione semicontinua inferiormente (superiormente) sulla curva $C_{0}$, se, preso un numero $\varepsilon>0$ ad arbitrio, è sempre possibile di determinarne un altro $\rho>0$ tale che la disuguaglianza

$$
I_{C}>I_{C_{0}}-\varepsilon
$$

$\left.{ }^{6}\right)$ Non si confonda con l'integrale definito, nel senso di integrale esteso ad un campo determinato.

7) Tale cioe che se $P_{1}^{\prime}$ e $P_{2}^{\prime}$ sono due punti di $C^{\prime}$ corrispondenti a $P_{1}$ e $P_{2}$ di $C, P_{1}^{\prime}$ preceda o segua $P_{2}^{\prime}$ su $C^{\prime}$ secondo che $P$ precede o segue $P_{2}$ su $C$. 
sia verificata per tutte le curve $C$ che appartengono ordinatamente all'intorno ( $\rho$ ) della $C_{0}$.

Si dird poi che $I_{C}$ è funzione continua sulla curva $C_{o}$ se, in luogo della disuguaglianza precedente, è verificata l'altra

$$
\left|I_{C}-I_{C_{0}}\right|<\varepsilon
$$

vale a dire se $I_{C}$ è, nello stesso tempo, funzione semicontinua inferiormente e superiormente, su $C_{0}$.

\section{$§ 2$.}

\section{Proposizioni preliminari.}

8. Lemma fondamentale. - Sia $f(x)$ una funzione data su un intervallo $(a, b)$, $e$ ivi integrabile e sempre inferiore in modulo ad un numero $M$.

Fissato un numero positivo $D$ e.preso ad arbitrio in $\varepsilon>0$, è sempre possibile di determinare un altro numero positivo $n$ tale che sia

$$
\left|\sum_{r} \int_{a_{r}}^{b_{r}} y^{\prime}(x) f(x) d x\right|<\varepsilon+M \sum_{r}\left|y\left(b_{r}\right)-y\left(a_{r}\right)\right|,
$$

per ogni sistema di intervalli $\left(a_{r}, b_{r}\right)$, non sourapponentisi, di $(a, b)$ e per ogni funzione $y(x)$ soddisfacente, su tutti questi intervalli, alla disuguaglianza $|y(x)| \leqslant n$ e quasi ovunque ${ }^{8}$ ), sugli stessi intervalli, all altra $\left|y^{\prime}(x)\right| \leq D^{9}$ ).

Adottando il modo di rappresentazione di una funzione di una variabile indicato da Landau e De la Vallée Poussin ${ }^{\text {to }}$ ), possiamo, per ogni valore dell'intero $n$, costruire un polinomio $P_{n}(x)$ soddisfacente alle due condizioni

$$
P_{n}(x) \underset{n \rightarrow \infty}{\longrightarrow} f(x)
$$

8) Quasi ovunque o quasi dappertutto indicano che la proprietà in questione ha luogo dappertutto ad eccezione al più di un insieme di misura nulla.

9) Questo lemma fu già dimostrato, in condizioni più generale da un lato e più restrittive da un altro, in loc. cit. ${ }^{4}$ ), pp. $241-243$.

$\left.{ }^{10}\right)$ E. Landau, Ueber die Approximation einer stetigen Funktion durch eine ganze rationale Funktion [Rendiconti Circolo Matematico di Palermo, t. XXV (1908), pp. 337-345]; CH. J. DE LA Vallée Poussin, Sur l'approximation des fonctions d'une variable réelle et de leurs derivées par des polynomes et des suites limitées de Fourier (Bulletins de la classe des Sciences de l'Académie Royale de Belgique, Igo8, pp. 193-254); F. Ryesz, Ueber die Approximation einer Funktion durch Polynome [Jahresbericht der Deutschen Mathematiker-Vereinigung, Bd. XVII (1908), pp. 196-2II]. 
quasi ovunque su $(a, b)$, e

$$
\left|P_{n}(x)\right| \leq M
$$

su tutto $(a, b)$. Cio premesso, osserviamo che si ha

$$
\left|\int_{a_{r}}^{b_{r}} y^{\prime}(x)\left\{f(x)-P_{n}(x)\right\} d x\right| \leqslant \int_{a_{r}}^{b_{r}}\left|y^{\prime}\right|\left|f-P_{n}\right| d x \leqslant D \int_{a_{r}}^{b_{r}}\left|f-P_{n}\right| d x
$$

e che, in forza di un noto teorema sullintegrazione per serie, $\dot{e}$, per un conveniente valore $n_{1}$,

$$
\int_{a}^{b}\left|f-P_{n_{1}}\right| d x<\frac{\varepsilon}{2 D} \text {. }
$$

Possiamo scrivere, pertanto,

$$
\begin{aligned}
\left|\sum_{r} \int_{a_{r}}^{b_{r}} y^{\prime} f(x) d x\right| & \leq \sum_{r}\left|\int_{a_{r}}^{b_{r}} y^{\prime}\left(f-P_{n_{\mathrm{I}}}\right) d x\right|+\left|\sum_{r} \int_{a_{r}}^{b_{r}} y^{\prime} P_{n_{\mathrm{I}}} d x\right| \\
& <\frac{\varepsilon}{2}+\left|\sum_{r} \int_{a_{r}}^{b_{r}} y^{\prime} P_{n_{\mathrm{r}}} d x\right| .
\end{aligned}
$$

Trasformiamo, con un'integrazione per parti, l'integrale di $y^{\prime} P_{n_{\mathrm{I}}}$ :

$$
\int_{a_{r}}^{b_{r}} y^{\prime} P_{n_{\mathrm{r}}} d x=\left[y\left(b_{r}\right)-y\left(a_{r}\right)\right] P_{n_{\mathrm{r}}}\left(b_{r}\right)-\int_{a_{r}}^{b_{r}}\left[y(x)-y\left(a_{r}\right)\right] P_{n_{\mathrm{I}}}^{\prime}(x) d x .
$$

Dunque, se $\dot{e}$, in tutti gli intervalli $\left(a_{r}, b_{r}\right),|y(x)| \leq n$, e se $\Pi$ indica il massimo modulo di $P_{n_{1}}^{\prime}$ su tutto l'intervallo $(a, b)$, abbiamo

$$
\left|\sum_{r} \int_{a_{r}}^{b_{r}} y^{\prime} P_{n_{\mathrm{I}}} d x\right|<M \sum_{r}\left|y\left(b_{r}\right)-y\left(a_{r}\right)\right|+2 n \Pi(b-a) .
$$

ed anche, quando sia

$$
\begin{gathered}
n<\frac{\varepsilon}{4 \Pi(b-a)}, \\
\left|\sum_{r} \int_{a_{r}}^{b_{r}} y^{\prime}(x) f(x) d x\right|<\varepsilon+M \sum_{r}\left|y\left(b_{r}\right)-y\left(a_{r}\right)\right| .
\end{gathered}
$$

9. Stabiliamo ora una formola importante per il seguito. Sia $(x y)$ un punto qualunque del campo $A$, e $\theta_{0}$ indichi la misura di un angolo qualsiasi. Abbiamo, per l'omogeneità dalla funzione $F$,

$$
F(x, y, \cos \theta, \operatorname{sen} \theta)=\cos \theta F_{x^{\prime}}(x, y, \cos \theta, \operatorname{sen} \theta)+\operatorname{sen} \theta F_{y^{\prime}}(x, y, \cos \theta, \operatorname{sen} \theta),
$$


e quindi, togliendo da ambo i membri di questa uguaglianza l'espressione

$$
\begin{gathered}
\cos \theta F_{x^{\prime}}\left(x, y, \cos \theta_{0}, \operatorname{sen} \theta_{0}\right)+\operatorname{sen} \theta F_{y^{\prime}}\left(x, y, \cos \theta_{0}, \operatorname{sen} \theta_{0}\right) \\
F(x, y, \cos \theta, \operatorname{sen} \theta)-\left\{\cos \theta F_{x^{\prime}}\left(x, y, \cos \theta_{0}, \operatorname{sen} \theta_{0}\right)+\operatorname{sen} \theta F_{y^{\prime}}\left(x, y, \cos \theta_{0}, \operatorname{sen} \theta_{0}\right)\right\} \\
=\cos \theta\left\{F_{x^{\prime}}(x, y, \cos \theta, \operatorname{sen} \theta)-F_{x^{\prime}}\left(x, y, \cos \theta_{0}, \operatorname{sen} \theta_{0}\right)\right\} \\
+\operatorname{sen} \theta\left\{F_{x^{\prime}}(x, y, \cos \theta, \operatorname{sen} \theta)-F_{y^{\prime}}\left(x, y, \cos \theta_{0}, \operatorname{sen} \theta_{0}\right)\right\} \\
=\cos \theta \int_{\theta_{0}}^{\theta} \frac{d}{d \alpha} F_{x^{\prime}}(x, y, \cos \alpha, \operatorname{sen} \alpha) d \alpha+\operatorname{sen} \theta \int_{\theta_{0}}^{\theta} \frac{d}{d \alpha} F_{y^{\prime}}(x, y, \cos x, \operatorname{sen} \alpha) d \alpha \\
=\cos \theta \int_{\theta_{o}}^{\theta}\left\{-F_{x^{\prime} x^{\prime}}(x, y, \cos \alpha, \operatorname{sen} \alpha) \operatorname{sen} \alpha+F_{x^{\prime} y^{\prime}}(x, y, \cos \alpha, \operatorname{sen} \alpha) \cos \alpha\right\} d \alpha \\
+\operatorname{sen} \theta \int_{\theta_{0}}^{\theta}\left\{-F_{y^{\prime} x^{\prime}}(x, y, \cos \alpha, \operatorname{sen} \alpha) \operatorname{sen} \alpha+F_{y^{\prime} y^{\prime}}(x, y, \cos \alpha, \operatorname{sen} \alpha) \cos \alpha\right\} d \alpha_{0}
\end{gathered}
$$

Introducendo qui la funzione $F_{\mathrm{I}}$ definita nell'introduzione:

$$
F_{\mathrm{r}} \equiv \frac{F_{x^{\prime} x^{\prime}}}{y^{\prime 2}} \equiv-\frac{F_{x^{\prime} y^{\prime}}}{x^{\prime} y^{\prime}} \equiv \frac{F_{y^{\prime} y^{\prime}}}{x^{\prime 2}}
$$

abbiamo, riunendo tutto in un solo integrale, $F(x, y, \cos \theta, \operatorname{sen} \theta)-\left\{\cos \theta F_{x}\left(x, y, \cos \theta_{o}, \operatorname{sen} \theta_{o}\right)+\operatorname{sen} \theta F_{y^{\prime}}\left(x, y, \cos \theta_{0}, \operatorname{sen} \theta_{0}\right)\right\}$

$$
=\int_{\theta_{0}}^{\theta}\{-\cos \theta \operatorname{sen} \alpha+\operatorname{sen} \theta \cos \alpha\} F_{1}(x, y, \cos \alpha, \operatorname{sen} \alpha) d \alpha,
$$

ed infine

$$
\begin{aligned}
F(x, y, \cos \theta, \operatorname{sen} \theta) & =\left\{\cos \theta F_{x^{\prime}}\left(x, y, \cos \theta_{0}, \operatorname{sen} \theta_{0}\right)+\operatorname{sen} \theta F_{y^{\prime}}\left(x, y, \cos \theta_{0}, \operatorname{sen} \theta_{0}\right)\right\} \\
& +\int_{\theta_{0}}^{\theta} F_{x}(x, y, \cos \alpha, \operatorname{sen} \alpha) \operatorname{sen}(\theta-\alpha) d \alpha,
\end{aligned}
$$

che è la formula a cui volevamo giungere e che è dovuta a Schwarz ${ }^{\text {II }}$ ).

$$
\$ 3 \text {. }
$$

\section{Condizioni sufficienti per la semicontinuità.}

a) II caso quasi-regolare definito.

10. Cominciamo con l'osservare che, nel caso quasi regolare definito, deve aversi sempre, per tutti i punti $(x y)$ di $A$ e per tutte le coppie $\left(x^{\prime} y^{\prime}\right)$ di numeri non am- 
bedue nulli,

oppure sempre

$$
F_{1} \geq 0, \quad F>0,
$$$$
F_{1} \leq \mathrm{o}, \quad F<\mathrm{o},
$$

non potendo essere verificate sempre e contemporaneamente le due disuguaglianze $F_{\mathrm{r}} \supseteq \mathrm{o}, F<\mathrm{o}$, e neppure le altre due $F_{\mathrm{r}} \leq \mathrm{o}, F>0{ }^{\mathrm{r} 2}$ ). Ciò detto, dimostriamo la seguente proposizione:

L'integrale quasi-regolare definito $\dot{e}$ una funzione semicontinua della curva d'integrazione: e precisamente, semicontinua inferiormente se sono soddisfatte le disuguaglianze (I) (integrale quasi-regolare definito positivo), superiormente se lo sono le (II) (integrale quasi-regolare definito negativo).

Occupiamoci del caso dell'integrale quasi regolare definito positivo: l'altro si deduce immediatamente da questo con il semplice cambiamento da $F$ in $-F$, che muta anche $F_{1}$ in $-F_{1}$.

Sia $C_{\mathrm{o}}$ una delle curve di cui si è detto al $\mathrm{n}^{\circ}$ 3. Preso su di essa, come origine degli archi, il primo estremo od un punto arbitrariamente scelto, nel caso di una curva chiusa, sia

$$
x=x_{0}(s), \quad y=y_{0}(s), \quad\left(0 \leq s \leq L_{0}\right)
$$

la sua rappresentazione analitica in funzione dell'arco s. Dividiamo la $C_{o}$ in $n$ parti, tutte di ugual lunghezza $\frac{\mathrm{I}}{n} L_{\mathrm{o}}$, mediante i punti $P_{\mathrm{o}}^{(0)}, P_{\mathrm{r}}^{(0)}, P_{2}^{(0)}, \ldots, P_{n}^{(0)}$, susseguen. tisi sulla curva nell'ordine fissato dal verso della curva stessa, e in modo che $P_{0}^{(o)}$ coincida con l'origine degli archi. Siano

$$
s_{0}=0<s_{1}<s_{2}<\cdots<s_{n}=L_{0},
$$

i valori di $s$ corrispondenti a tali punti. Sarà

$$
s_{i+1}-s_{i}=\frac{\mathrm{I}}{n} L_{\mathrm{o}} \quad(i=0, \mathrm{I}, 2, \ldots, n-\mathrm{I}) .
$$

Indichiamo con $\rho$ un numero positivo minore $\mathrm{di} \frac{\mathrm{I}}{n^{2}}$ e tale che ogni curva appartenente ordinatamente all'intorno $(\rho)$ di uno qualunque degli archi $\left.C_{\mathrm{o}}\left(P_{i}^{(\mathrm{o})}, P_{i+\mathrm{I}}^{(\mathrm{o})}\right){ }^{\mathrm{I3}}\right)$ di $C_{o}$ abbia lunghezza superiore a $\frac{I}{2 n} L_{0}$; e consideriamo una qualunque curva $C$ $\left(n^{\circ} 3\right)$ che appartenga ordinatamente allintorno $(\rho)$ della $C_{0}$. Tra le corrispondenze

${ }^{2}$ ) Ctr. loc. cit. I), p. 302.

I3) $P_{i}^{(0)}$ e $P_{i+1}^{(0)}$ sono gli estremi dell'arco considerato. 
biunivoche, ordinate e continue esistenti fra i punti della $C$ e della $C_{0}$, e tali che la distanza fra i punti corrispondenti risulti sempre inferiore a $P$, scegliamone una $e$ indichiamo con $P_{0}, P_{1}, P_{2}, \ldots, P_{n}$ i punti della $C$ che vengono cosi a corrispondere ordinatamente ai punti $P_{0}^{(0)}, P_{1}^{(0)}, P_{2}^{(0)}, \ldots, P_{n}^{(0)}$ della $C_{0}$. L'arco $C\left(P_{i}, P_{i+1}\right)$ appartiene allora ordinatamente all'intorno $(\hat{p})$ dell'arco $C_{0}\left(P_{i}^{(o)}, P_{i+\mathrm{I}}^{(0)}\right)$, e per l'ipotesi fatta sopra sul numero $\rho$, la lunghezza del primo risulta superiore a $\frac{\mathrm{I}}{2 n} L_{0}$, cioè alla meta di quella del secondo. Contiamo le lunghezze degli archi della $C$ a partire dal punto $P_{o}$ e indichiamole genericamente con la lettera $\sigma$; chiamiamo poi $\sigma_{1}, \sigma_{2}, \ldots, \sigma_{n}$, le lunghezze degli archi $C\left(P_{0}, P_{1}\right), C\left(P_{0}, P_{2}\right), \ldots, C\left(P_{0}, P_{n}\right)$. Per quanto si è detto or ora, è

$$
\sigma_{i+1}-\sigma_{i}>\frac{s_{i+1}-s_{i}}{2} \quad(i=0,1, \ldots, n-1)
$$

Confrontiamo $i$ valori dei due integrali

$$
\int_{C_{0}\left(P_{i}^{(0)}, P_{i+1}^{(0)}\right)} F d s, \quad \int_{C\left(P_{i}, P_{i+1}\right) .} F d \sigma .
$$

A tal uopo, considerato un qualsiasi cerchio del piano $(x y)$ contenente la curva $C_{\text {o }}$ ed almeno tutti $i$ punti dello stesso piano distanti da essa di non più di un numero positivo qualunque maggiore di $p^{15}$ ), indichiamo con $m$ e $M$ il minimo e il massimo della funzione $F$ nell'insieme (chiuso) dei punti di $A$ che appartengono al cerchio detto e per tutte le coppie $\left(x^{\prime}, y^{\prime}\right)$ soddisfacenti all'equazione $x^{\prime 2}+y^{\prime 2}=1$. Per la seconda delle (I) è $m>0$.

Avendosi

si ha che, se è

$$
\begin{aligned}
& \int_{C_{\mathrm{o}}\left(p_{i}^{(0)}, p_{i+1}^{(0)}\right)} F d s \leq M\left(s_{i+1}-s_{i}\right) \\
& \int_{C\left(P_{i}, p_{i+1}\right)} F d \sigma \supseteq m\left(\sigma_{i+1}-\sigma_{i}\right)
\end{aligned}
$$

è senz'altro,

$$
\sigma_{i+\mathrm{r}}-\sigma_{i} \supset \frac{M}{m}\left(s_{i+\mathrm{r}}-s_{i}\right)
$$

(3)

$$
\int_{C\left(P_{i}, P_{i+1}\right)} F d \sigma \supseteq \int_{C_{\mathrm{o}}\left(P_{i}^{(0)}, P_{i+1}^{(0)}\right)} F d s .
$$

14) Poniamo $\sigma_{\mathrm{o}}=0$.

r5) Intendiamo per distanza di un punto da una curva il minimo delle distanze del punto stesso dai punti della curva.

Rend. Circ. Matem. Palermo, t. XLIV (1920). - Stampato it is settembre 1920. 
Consideriamo il caso in cui si abbia

$$
\sigma_{i+1}-\sigma_{i}<\frac{M}{m}\left(s_{i+1}-s_{i}\right) .
$$

Poniamo fra $i$ due archi $C_{o}\left(P_{i}^{(0)}, P_{i+1}^{(0)}\right), C\left(P_{i}, P_{i+1}\right)$ una nuova corrispondenza biunivoca, ordinata e continua, definita dalla relazione lineare

$$
\sigma=\sigma_{i}+\frac{\sigma_{i+1}-\sigma_{i}}{s_{i+1}-s_{i}}\left(s-s_{i}\right)
$$

Abbiamo, per la (I) e la (4),

$$
\frac{\mathrm{I}}{2}<\frac{\sigma_{i+1}-\sigma_{i}}{s_{i+1}-s_{i}}<\frac{M}{m} .
$$

Scegliendo $s$ come parametro di una rappresentazione analitica simultanea degli archi $C_{o}\left(P_{i}^{(0)}, P_{i+1}^{(0)}\right), C\left(P_{i}, P_{t+1}\right)$, si avranno, per il primo, le equazioni

e per il secondo

$$
x=x_{0}(s), \quad y=y_{0}(s), \quad\left(s_{i} \leqslant s \leqslant s_{i+1}\right),
$$

$$
x=x(s), \quad y=y(s), \quad\left(s_{i} \leq s \leq s_{i+1}\right) ;
$$

e sarà quasi ovunque sull'intervallo $\left(s_{i}, s_{i+s}\right)$

$$
\begin{cases}\left|x_{\mathrm{o}}^{\prime}(s)\right| \leq \mathrm{I}, & \left|y_{\mathrm{o}}^{\prime}(s)\right| \leq \mathrm{i}, \\ \left|x^{\prime}(s)\right|<\frac{M}{m}, & \left|y^{\prime}(s)\right|<\frac{M}{m} .\end{cases}
$$

Detti poi $P^{(0)}$ e $P$ i punti di $C_{0}$ e $C$ corrispondenti ad uno stesso valore $s$ qualsiasi di $\left(s_{i}, s_{i+1}\right)$, e $P_{(0)}$ il punto di $C_{o}$ corrispondente a $P$ nella prima corrispondenza considerata fra $C_{\mathrm{o}}$ e $C$, la distanza fra $P_{(0)}$ e $P$ risulta minore di $\rho<\frac{\mathrm{I}}{n^{2}}$ e quella fra $P_{(0)}$ e $P^{(0)}$, minore di $\frac{L_{0}}{n}$ (perchè questi due punti appartengono ad uno stesso arco di $C_{\mathrm{o}}$ di lunghezza uguale a $\left.\frac{L_{\mathrm{o}}}{n}\right)$. La distanza fra $P^{(0)}$ e $P$ è dunque minore di $\frac{\mathrm{I}+L_{\mathrm{o}}}{n}$ e si ha

$$
\left|x(s)-x_{0}(s)\right|<\frac{I+L_{0}}{n}, \quad\left|y(s)-y_{0}(s)\right|<\frac{I+L_{0}}{n}
$$

per tutti gli $s$ di $\left(s_{i}, s_{i+1}\right)$. Di più è

$$
\left\{\begin{array}{l}
\left|x\left(s_{i}\right)-x_{0}\left(s_{i}\right)\right|<\rho, \quad\left|y\left(s_{i}\right)-y_{0}\left(s_{i}\right)\right|<\rho, \\
\left|x\left(s_{i+1}\right)-x_{0}\left(s_{i+1}\right)\right|<\rho, \quad\left|y\left(s_{i+1}\right)-y_{0}\left(s_{i+1}\right)\right|<\rho,
\end{array}\right.
$$

perchè i punti $P_{i}^{(0)}, P_{i}$ distano fra loro per meno di $\rho$, e ugualmente $P_{i+1}^{(0)}, P_{i+1}$. 
Consideriamo la differenza fra i due integrali (2) e scriviamola nel seguente modo:

$$
\begin{gathered}
D_{i}=\int_{C\left(P_{i}, p_{i+1}\right)} F d \sigma-\int_{C_{0}\left(P_{i}^{(0)}, p_{i+1}^{(0)}\right)} F d s \\
=\int_{s_{i}}^{s_{i+s}}\left\{F\left(x(s), y(s), x^{\prime}(s), y^{\prime}(s)\right)-F\left(x_{0}(s), y_{0}(s), x_{0}^{\prime}(s), y_{0}^{\prime}(s)\right)\right\} d s \\
=\int_{s_{i}}^{s_{i+1}}\left\{F\left(x, y, x^{\prime}, y^{\prime}\right)-F\left(x_{0}, y_{0}, x^{\prime}, y^{\prime}\right)\right\} d s \\
+\int_{s_{i}}^{s_{i+1}}\left\{F\left(x_{0}, y_{0}, x^{\prime}, y^{\prime}\right)-F\left(x_{0}, y_{0}, x_{0}^{\prime}, y_{0}^{\prime}\right)\right\} d s .
\end{gathered}
$$

Osserviamo qul che, avendosi

$$
x^{\prime}(s)=\frac{d x}{d \sigma} \cdot \frac{\sigma_{i+1}-\sigma_{i}}{s_{i+1}-s_{i}}, \quad y^{\prime}(s)=\frac{d y}{d \sigma} \cdot \frac{\sigma_{i+1}-\sigma_{i}}{s_{i+1}-s_{i}},
$$

è, per la (s), quasi ovunque su $\left(s_{i}, s_{i+1}\right)$,

$$
\frac{\mathrm{I}}{4}<{\overline{x^{\prime}(s)}}^{2}+{\overline{y^{\prime}(s)}}^{2}<\left(\frac{M}{m}\right)^{2} \text {. }
$$

Preso dunque ad arbitrio un numero positivo $\varepsilon$, possiamo, per la continuitd della funzione $F$, determinarne un altro $\delta$ tale che, essendo $\left(x_{0}, y_{0}\right)$ un qualsiasi punto di $C_{\mathrm{o}}$ e $(x y)$ un altro punto qualunque di $A$ tale però da avere una distanza da $\left(x_{0} y_{0}\right)$ non superiore a $\delta$, ed essendo verificata la doppia disuguaglianza

si abbia

$$
\frac{1}{4} \leqslant x^{\prime 2}+y^{\prime 2} \leqslant\left(\frac{M}{m}\right)^{2}
$$

$$
\left|F\left(x, y, x^{\prime}, y^{\prime}\right)-F\left(x_{0}, y_{0}, x^{\prime}, y^{\prime}\right)\right|<\varepsilon .
$$

Ne viene perciò che, per ogni $n$ tale che sia.

$\dot{e}$, in forza della (7),

$$
\frac{\mathrm{I}+L_{\mathrm{o}}}{n}<\frac{\mathrm{I}}{2} \delta
$$

$$
\int_{s_{i}}^{s_{i+1}}\left\{F\left(x, y, x^{\prime}, y^{\prime}\right)-F\left(x_{0}, y_{0}, x^{\prime}, y^{\prime}\right)\right\} d s>-\varepsilon\left(s_{i+1}-s_{i}\right) .
$$

Per la formula stabilita al n. 9 si ha poi, quasi dappertutto in $\left(s_{i}, s_{i+1}\right)$,

$$
\begin{gathered}
F\left(x_{0}, y_{0}, x^{\prime}, y^{\prime}\right)=\left\{x^{\prime} F_{x^{\prime}}\left(x_{0}, y_{0}, x_{0}^{\prime}, y_{0}^{\prime}\right)+y^{\prime} F_{y^{\prime}}\left(x_{0}, y_{0}, x_{0}^{\prime}, y_{0}^{\prime}\right)\right\} \\
\quad+\sqrt{x^{\prime 2}+y^{\prime 2}} \int_{\theta_{0}}^{\theta} F_{1}\left(x_{0}, y_{0}, \cos \alpha, \operatorname{sen} \alpha\right) \operatorname{sen}(\theta-\alpha) d \alpha,
\end{gathered}
$$


dove $\theta_{\mathrm{o}}$ e $\theta$ sono definiti dalle uguaglianze

e perciò

$$
\begin{array}{ll}
x_{0}^{\prime}=\cos \theta_{0}, & y_{0}^{\prime}=\operatorname{sen} \theta_{o} \\
x^{\prime}=\sqrt{x^{\prime 2}+y^{\prime 2}} \cos \theta, & y^{\prime}=\sqrt{x^{\prime 2}+y^{\prime 2}} \operatorname{sen} \theta ;
\end{array}
$$

$$
\begin{aligned}
& F\left(x_{0}, y_{0}, x^{\prime}, y^{\prime}\right)-F\left(x_{0}, y_{0}, x_{0}^{\prime}, y_{0}^{\prime}\right)=\left\{\left(x^{\prime}-x_{0}^{\prime}\right) F_{x^{\prime}}\left(x_{0}, y_{0}, x_{0}^{\prime}, y_{0}^{\prime}\right)\right. \\
& \left.+\left(y^{\prime}-y_{0}^{\prime}\right) F_{y^{\prime}}\left(x_{0}, y_{0}^{\prime}, x_{0}, y_{0}^{\prime}\right)\right\}+\sqrt{x^{\prime 2}+y^{\prime 2}} \int_{\theta_{0}}^{\theta} F_{1}\left(x_{0}, y_{0}, \cos \alpha, \operatorname{sen} \alpha\right) \operatorname{sen}(\theta-\alpha) d x .
\end{aligned}
$$

Tenendo conto di questa uguaglianza, della prima delle disuguaglianze (I) e della (Io), si ha

$D_{i}>-\varepsilon\left(s_{i+1}-s_{i}\right)+\int_{s_{i}}^{s_{i+\varepsilon}}\left\{\left(x^{\prime}-x_{0}^{\prime}\right) F_{x^{\prime}}\left(x_{0}, y_{0}, x_{0}^{\prime}, y_{0}^{\prime}\right)+\left(y^{\prime}-y_{0}^{\prime}\right) F_{y^{\prime}}\left(x_{0}, y_{0}, x_{0}^{\prime}, y_{0}^{\prime}\right)\right\} d s$.

Sommando ora rispetto all'indice $i$ e tenendo conto della disuguaglianza (3), valida quando non sia soddisfatta la (4), abbiamo

$$
\begin{gathered}
\int_{C} F d \sigma-\int_{C_{0}} F d s=\sum_{i} D_{i}>-\varepsilon L_{0} \\
+\sum_{i}^{\prime} \int_{s_{I}}^{s_{i+1}}\left\{\left(x^{\prime}-x_{0}^{\prime}\right) F_{x^{\prime}}\left(x_{0}, y_{0}, x_{0}^{\prime}, y_{0}^{\prime}\right)+\left(y^{\prime}-y_{0}^{\prime}\right) F_{y^{\prime}}\left(x_{0}, y_{0}, x_{0}^{\prime}, y_{0}^{\prime}\right)\right\} d s,
\end{gathered}
$$

intendendo che la sommatoria $\Sigma^{\prime}$ sia estesa solamente a quegli archi per i quali vale la (4). Questa sommatoria puó essere decomposta nelle due

$$
\sum_{i}^{\prime} \int_{s_{i}}^{s_{i+1}}\left(x^{\prime}-x_{0}^{\prime}\right) F_{x^{\prime}}\left(x_{0}, y_{0}, x_{0}^{\prime}, y_{0}^{\prime}\right) d s_{,}, \sum_{i}^{\prime} \int_{s_{i}}^{s_{i+1}}\left(y^{\prime}-y_{0}^{\prime}\right) F_{y^{\prime}}\left(x_{0}, y_{0}, x_{0}^{\prime}, y_{0}^{\prime}\right) d s,
$$

a ciascuna delle quali puó applicarsi il lemma del $\$ 2$. Ed infatti, le funzioni $F_{x^{\prime}}\left(x_{0}, y_{0}, x_{0}^{\prime}, y_{0}^{\prime}\right), F_{y^{\prime}}\left(x_{0}, y_{0}, x_{0}^{\prime}, y_{0}^{\prime}\right)$ sono integrabili e inferiori in modulo ad un numero $M_{1}$; gli intervalli $\left(s_{i}, s_{i+1}\right)$ non si sovrappongono e appartengono a $\left(\mathrm{o}, L_{\mathrm{o}}\right)$; le funzioni $x(s)-x_{0}(s), y(s)-y_{0}(s)$ soddisfano, sugli intervalli $\left(s_{i}, s_{i+1}\right)$ che figurano nelle $\Sigma^{\prime}$ alle disuguaglianze $(7)$ ed alle $(6)$ e quindi anche alle

$$
\left|x^{\prime}-x_{0}^{\prime}\right|<\mathrm{I}+\frac{M}{m}, \quad\left|y^{\prime}-y_{\mathrm{o}}^{\prime}\right|<\mathrm{I}+\frac{M}{m} .
$$

Prendendo dunque l'intero $n$ soddisfacente alla (9) e sufficientemente grande, abbiamo, per il lemma ricordato,

$\left|\sum_{i}^{\prime} \int_{s_{i}}^{s_{i+1}}\left(x^{\prime}-x_{0}^{\prime}\right) F_{x^{\prime}}\left(x_{0}, y_{0} ; x_{0}^{\prime}, y_{0}^{\prime}\right) d s\right|<\varepsilon+M_{1} \sum_{i}^{\prime}\left|x\left(s_{i+1}\right)-x_{0}\left(s_{i+1}\right)-x\left(s_{i}\right)+x_{0}\left(s_{i}\right)\right|$

$\left|\sum_{i}^{\prime} \int_{s_{i}}^{s_{i+1}}\left(y^{\prime}-y_{0}^{\prime}\right) F_{y^{\prime}}\left(x_{0}, y_{0}, x_{0}^{\prime}, y_{0}^{\prime}\right) d s\right|<\varepsilon+M_{1} \sum_{i}^{\prime}\left|y\left(s_{i+1}\right)-y_{0}\left(s_{i+1}\right)-y\left(s_{i}\right)+y_{0}\left(s_{i}\right)\right|$, 
e, tenendo conto delle disuguaglianze $(8)$ e dell'ipotesi $p<\frac{\mathrm{I}}{n^{2}}$,

$$
\left|\sum_{i}^{\prime} \int_{s_{i}}^{s_{i+1}}\left\{\left(x^{\prime}-x_{0}^{\prime}\right) F_{x^{\prime}}+\left(y^{\prime}-y_{0}^{\prime}\right) F_{y^{\prime}}\right\} d s\right|<2 \varepsilon+4 M_{1} p n<2 \varepsilon+\frac{4 M_{1}}{n},
$$

e se $n$ è sufficientemente grande,

$$
<3 \varepsilon .
$$

E dunque, per $n$ abbastanza grande,

$$
\int_{C} F d \sigma-\int_{C_{0}} F d s>-\varepsilon\left(L_{0}+3\right) .
$$

Siccome $\varepsilon$ è stato scelto ad arbitrio e questa disuguaglianza vale per tutte le curve $C$ dell'intorno $(\rho)$ della $C_{0}$, intorno scelto nel modo indicato, la semicontinuita inferiore di $I_{C}$ sulla $C_{o}$ è dimostrata ${ }^{\mathrm{x}}$ ).

$$
\$ 4 \text {. }
$$

\section{Condizioni sufficienti per la semicontinuità.}

$$
\text { b). - II caso quasi-regolare. }
$$

11. Dimostriamo che:

Se, in tutto il campo $A$ e per tutte le coppie $\left(x^{\prime}, y^{\prime}\right)$ di numeri non nulli insieme, si ha sempre $F_{1}\left(x, y, x^{\prime}, y^{\prime}\right) \geqslant 0$, senza però che per nessun punto $(\bar{x}, \bar{y})$ di $A$ sia $F_{1}\left(\bar{x}, \bar{y}, x^{\prime}, y\right)=0$ identicamente per tutte le coppie $\left(x^{\prime}, y^{\prime}\right)$ ammesse, l'integrale $\int_{C} F d s$ è una funzione semicontinua inferiormente (superiormente se, invece, della $F_{\mathrm{r}} \supseteq 0$, si ba $\left.F_{1} \leq 0\right)$.

16) Il Goursat [loc. cit. ${ }^{2}$ ), $n^{0}$ 3] nel dimostrare, per le curve ordinarie, la proposizione qui stabilita in generale, dopo aver notato che basta all'uopo confrontare il valore dell'integrale calcolato su una curva fissa $C$ solamente con quello relativo alle curve variabili $C^{\prime}$ aventi lunghezza sempre inferiore ad un numero fisso, utilizza la seguente osservazione: "Étant donnée une suite de courbes $C^{\prime}$ satisfaisant à cette condition (lunghezza inferiore ad un numero fisso), on peut alors faire un changement de la variable indépendante $t$ tel que les valeurs absolues des dérivées $x^{\prime}, y^{\prime}, \xi_{\prime}^{\prime}, \eta^{\prime}$ (dove $x=x(t), y=y(t),(a \leq t \leq b)$, rappresentano le equazioni parametriche della curva $C$ e $x=x(t)+\xi(t)$, $y=y(t)+n(t)$ quelle della $C^{\prime}$ ) restent bornées dans l'intervalle $(a, b)$ ». Poiche, dopo questo cambiamento della variabile indipendente, te funzioni $\xi$ e $\eta$ devono continuare a tendere uniformemente a 
Osserviamo, innanzitutto, che, preso comunque un punto $P_{\mathrm{o}}\left(x_{\mathrm{o}}, y_{\mathrm{o}}\right)$ nel campo $A$, si possono sempre scegliere tre costanti $p_{0}, q_{0}$ e $r_{0}$, con $r_{0}<0$, in modo che la

zero in tutto $(a, b)$, noi vogliamo rilevare qui che la possibilita del cambiamento indicato non sussiste sempre.

Consideriamo, ad esempio, la curva $C$ definita dalle equazioni

$$
x=t, \quad y=0 ; \quad(0 \leq t \leq \mathrm{I})
$$

e la $C_{r, n}$, dove $r$ e $n$ indicano due qualsiasi numeri interi $\supseteq 2$, dalle altre

$$
\left.C_{r, n}\right) \quad x=t, \quad y=y_{r, n}(t), \quad(0 \leq t \leq \mathrm{I}),
$$

essendo

$$
\begin{gathered}
y_{r, n}(t)=0 \quad \text { per } \quad 0 \leq t \leq \frac{\mathrm{I}}{r}, \\
y_{r, n}(t)=\frac{\mathrm{I}}{r n} \sin \left\{r^{2} n^{2}\left(t-\frac{\mathrm{I}}{r}\right)\right\} \quad \text { per } \quad \frac{\mathrm{I}}{r} \leq t \leq \frac{\mathrm{I}}{r}+\frac{\mathrm{I}}{r n}, \\
y_{r, n}(t)=\frac{\mathrm{I}-t}{r n-n-\mathrm{I}} \sin r n \quad \text { per } \quad \frac{\mathrm{I}}{r}+\frac{\mathrm{I}}{r n} \leq t \leq \mathrm{I} .
\end{gathered}
$$

La funzione $y_{r, n}(t)$ ha derivata continua in tutto l'intervallo $(0,1)$ ad eccezione dei punti $t=\frac{1}{r}$, $t=\frac{\mathrm{I}}{r}+\frac{\mathrm{I}}{r n}$. Si può anche modificare leggermente la sua definizione nelle vicinanze di questi punti in modo che essa ammetta ovunque derivata continua; ma cio non ha importanza per il seguito.

Le curve $C_{r, n}$, considerate per tutti i possibili valori di $r$ e $n$, si possono ordinare in un'unica successione in molti modi. Scegliamo una di tali successioni, che chiameremo successione delle $C_{r, n}$.

E evidente che la successione delle $C_{r n}$ tende uniformemente alla curva $C$, perchè $y_{r, n}(t)$, quando $r n \leftrightarrow \infty$, tende a zero uniformemente su tutto l'intervallo $(0,1)$. Indicando poi con $L_{r, n}$ la lunghezza della $C_{r, n}$, si ha

$$
\begin{gathered}
L_{r n}=\frac{\mathrm{I}}{r}+\int_{\frac{\mathrm{I}}{r}}^{\frac{1}{r}+\frac{1}{r n}} \sqrt{\mathrm{I}+r^{2} n^{2} \cos ^{2}\left\{r^{2} n^{2}\left(t-\frac{\mathrm{I}}{r}\right)\right\}} d t+\sqrt{\frac{\mathrm{I}}{r^{2} n^{2}} \sin ^{2} r n+\left(\mathrm{I}-\frac{\mathrm{I}}{r}-\frac{\mathrm{I}}{r n}\right)^{2}}, \\
L_{r n}<\frac{1}{2}+2 \sqrt{2} .
\end{gathered}
$$

Poichè la derivata di $y_{r n}(t)$ in $\left(\frac{\mathrm{I}}{r}, \frac{\mathrm{I}}{r}+\frac{\mathrm{I}}{r n}\right)$ è data da

$$
y_{r n}^{\prime}(t)=r n \cos \left\{r n\left(t-\frac{\mathrm{I}}{r}\right)\right\}
$$

e non resta quindi in modulo inferiore ad un numero fisso per tutti gli $r$ e $n$, scegliamo comunque un'altra rappresentazione parametrica della $C$ e delle $C_{r n}$ tale che, essendo le rispettive equazioni

C)

$$
\begin{array}{lll}
x=\bar{x}(\tau), & y=\bar{y}(\tau) \equiv 0, & \\
x=\bar{x}_{r n}(\tau), & y=\bar{y}_{r n}(\tau), & (0 \leq \tau \leq \mathrm{I})
\end{array}
$$$$
\left.C_{r n}\right)
$$ 
funzione

$$
\vec{F}\left(x, y, x^{\prime}, y^{\prime}\right) \equiv F\left(x, y, x^{\prime}, y^{\prime}\right)+p_{0} x^{\prime}+q_{o} y^{\prime}
$$

le derivate delle funzioni $\bar{x}, \bar{y}, \bar{x}_{r n}, \bar{y}_{r n}$ restino tutte in modulo inferiori ad un numero fisso, che diremo $M$, in tutto l'intervallo $(0,1)$ e per tutti i valori di $r$ e $n$.

Dico che le $\bar{x}_{r n}, \bar{y}_{r n}$ non possono tendere uniformemente alle $\bar{x}, \bar{y}$, in tutto $(0,1)$.

$\mathrm{Si}$ supponga, infatti, che tale convergenza uniforme sussista e si indichino con $P_{r n}^{\prime}$ e $P_{r n}^{\prime \prime}$ i punti della curva $C_{r n}$ che hanno rispettivamente per coordinate

$$
\begin{array}{ll}
x=\frac{\mathrm{I}}{r}, & y=0, \\
x=\frac{\mathrm{I}}{r}+\frac{\mathrm{I}}{r n}, & y=\frac{\mathrm{I}}{r n} \operatorname{sen} r n .
\end{array}
$$

Tenendo fisso l'indice $r$ e facendo crescere all'infinito $n$, $i$ punti detti tendono entrambi al punto $P_{r}$ di $C$ che ha per coordinate $x=\frac{I}{r}, y=0$. Sia $\tau_{r}$ il valore del parametro $\tau$ che corrisponde al punto $P_{r}$, o più generalmente, poichè le funzioni $\bar{x}(\tau), \bar{y}(\tau)$ potrebbero restare costanti in tutto un intervallo di valori di $\tau$, si indichi con $\hat{\sigma}_{r}$ l'intervallo costituito da tutti $\mathrm{i}$ valori di $\tau$ che corrispondono al punto $P_{r}$ di $C$. Siano poi $\tau_{r, n}^{\prime}, \tau_{r, n}^{\prime \prime}$ dei valori di $\tau$ che corrispondono rispettivamente a $P_{r n}^{\prime}, P_{r n}^{\prime \prime}$. Tenuto inalterato l'indice $r$, i punti limiti dei punti $\tau_{r, n}^{\prime}, \tau_{r, n}^{\prime \prime}$, per $n \rightarrow \infty$, devono necessariamente appartenere all'intervallo $\delta_{r}$. Ed infatti, se $\tau_{r}^{\prime}$ è ad esempio un punto limite di $\tau_{r n}^{\prime}$, per $n \rightarrow \infty$, per la continuità delle funzioni $\bar{x}(\tau), \bar{y}(\tau)$ e per l'ammessa convergenza uniforme delle $\bar{x}_{r n}(\tau), \bar{y}_{r n}(\tau)$ alle precedenti funzioni, è $\bar{x}\left(\tau_{r}^{\prime}\right)=\lim \bar{x}_{r n}\left(\tau_{r n}^{\prime}\right)=\frac{\mathrm{I}}{r}, \bar{y}\left(\tau_{r}^{\prime}\right)=\lim \bar{y}_{r, n}\left(\tau_{r n}\right)=0$, vale a dire $\bar{x}\left(\tau_{r}^{\prime}\right)$, $\bar{y}\left(\tau_{r}^{\prime}\right)$ sono le coordinate di $P_{r}$. Si osservi ora che la differenza $\tau_{r n}^{\prime \prime}-\tau_{n}^{\prime}$ si mantiene, per tutti gli $n$, superiore ad un numero fisso maggiore di zero. Si ha inyero che la lunghezza dell'arco di $C_{r n}$ compreso fra i punti $P_{r n}^{\prime}$ e $P_{r n}^{\prime \prime}$ è espressa dall'integrale

$$
\int_{\tau_{r n}^{\prime}}^{\tau_{r n}^{\prime \prime}} \sqrt{\overline{x_{r n}^{\prime 2}+\bar{y}_{r n}^{\prime 2}}} d \tau
$$

il quale, per essere sempre $\left|\bar{x}_{r n}^{\prime}\right|<M,\left|\bar{y}_{r n}^{\prime}\right|<M$, è minore di $M \sqrt{2}\left(\tau_{r n}^{\prime \prime}-\tau_{r n}^{\prime}\right)$. D'altra parte, la stessa lunghezza è espressa anche dall'integrale

$$
\int_{\frac{1}{r}}^{\frac{1}{r}+\frac{1}{r n}} \sqrt{1+r^{2} n^{2} \cos ^{2}\left\{r^{2} n^{2}\left(t-\frac{1}{r}\right)\right\}} d t=\frac{1}{r^{2} n^{2}} \int_{0}^{r n} \sqrt{1+r^{2} n^{2} \cos ^{2} t} d t>\frac{1}{r n} \int_{0}^{r n}|\cos t| d t .
$$

Ora, se $k_{r n}$ indica un numero intero tale che sia

$$
\frac{\pi}{2} k_{r n} \leq r n<\frac{\pi}{2}\left(k_{r n}+1\right)
$$


sia maggiore di zero in tutti i punti di $A$ che appartengono al cerchio di centro $P_{\text {o }}$ e raggio $r_{0}$ e per tutte le coppie $\left(x^{\prime}, y^{\prime}\right)$ di numeri non ambedue nulli. Ed infatti, considerata la superficie conica

$$
z=F\left(x_{0}, y_{0}, x^{\prime}, y^{\prime}\right)
$$

riferita ad un sistema di assi cartesiani ortogonali $x^{\prime}, y^{\prime}$, avente l'origine nel vertice della superficie stessa, e detto $\left(\bar{x}^{\prime}, \bar{y}^{\prime}\right)$ un punto del piano $\left(x^{\prime}, y^{\prime}\right)$ per il quale sia $F_{1}\left(x_{0}, y_{0}, \overline{x^{\prime}}, y^{\prime}\right)>0$ e $F\left(x_{0}, y_{0}, \overline{x^{\prime}}, y^{\bar{\prime}}\right) \neq 0$, l'equazione del piano tangente alla superficie nel punto di coordinate $\bar{x}^{\prime}, \overline{y^{\prime}}, \bar{z}=F\left(x_{0}, y_{0}, \overline{x^{\prime}}, \overline{y^{\prime}}\right)$, ̀̀

$$
z=x^{\prime} F_{x^{\prime}}\left(x_{0}, y_{0}, \bar{x}^{\prime}, \bar{y}^{\prime}\right)+y^{\prime} F_{y^{\prime}}\left(x_{0}, y_{0}, \overline{x^{\prime}}, \overline{y^{\prime}}\right) \text {. }
$$

Questo piano tocca la superficie conica lungo tutta la generatrice passante per il punto

e quindi tale che

$$
\begin{gathered}
\frac{2 r n}{\pi}-1<k_{r n} \leq \frac{2 r n}{\pi} \\
\frac{1}{r n} \int_{0}^{r n}|\cos t| d t \gg \frac{1}{r n} k_{r n}>\frac{2}{\pi}-\frac{\mathrm{I}}{r n}>\frac{\mathrm{I}}{\pi} .
\end{gathered}
$$

Si ha dunque, in definitiva, she la lunghezza dell'arco considerato è compresa fra $\frac{I}{\pi} e$ $M \sqrt{2}\left(\tau_{r n}^{\prime \prime}-\tau_{r n}^{\prime}\right)$ e che perciò vale la disuguaglianza

$$
\tau_{n}^{\prime \prime}-\tau_{m}^{\prime}>\frac{\mathrm{I}}{\pi M \sqrt{2}},
$$

la quale è vera qualunque sia $r$ e qualunque sia $n$. Per quanto si è mostrato più sopra' sui punti limiti di $\tau_{r n}^{\prime}, \tau_{r n}^{\prime \prime}$, risulta percio che l'ampiezza di $\delta_{r}$ e $>\frac{I}{\pi M \sqrt{2}}$.

Osserviamo, infine, che due qualsiasi $\delta_{r}, \delta_{r_{1}}$ e $\delta_{r_{2}}$ ad esempio, sono intervalli completamente distinti di $(o, I)$ e cioè senza punti comuni. Ciò perchè nel primo è costantemente $\bar{x}(\tau)=\frac{\mathrm{I}}{r_{1}} \mathrm{e}$ nel secondo $\bar{x}(\tau)=\frac{1}{r_{2}}$. Deve esser pertanto $\sum_{r=2}^{\infty} \delta_{r}<\mathrm{I}$. Ma essendo sempre, come abbiamo dimostrato, $\delta_{r}>\frac{1}{\pi M \sqrt{2}}$, la serie $\sum \delta_{r}$ è divergente : si è giunti così ad una contraddizione la quale prova esser falsa l'ipotesi ammessa, che cioe le funzioni $\bar{x}_{r n}(\tau), \bar{y}_{r n}(\tau)$ tendano uniformemente alle $\bar{x}(\tau)$, $\bar{y}(\tau)$, in tutto l'intervallo $(0,1)$. 
$\left(\bar{x}^{\prime}, \overline{y^{\prime}}, \bar{z}\right)$ e non ha in comune con essa nessun altro punto. Ciò perché, ponendo

$$
\begin{array}{ll}
x^{\prime}=k \cos \theta, & y^{\prime}=k \operatorname{sen} \theta \\
\bar{x}^{\prime}=\bar{k} \cos \bar{\theta}, & \overline{y^{\prime \prime}}=\bar{k} \operatorname{sen} \bar{\theta},
\end{array}
$$

si ha, per la formula del $n^{0} 9$ :

$$
\begin{gathered}
F\left(x_{0}, y_{0}, x^{\prime}, y^{\prime}\right)-\left\{x^{\prime} F_{x^{\prime}}\left(x_{0}, y_{0}, \cos \bar{\theta}, \operatorname{sen} \bar{\theta}\right)+y^{\prime} F_{y^{\prime}}\left(x_{0}, y_{0}, \cos \bar{\theta}, \operatorname{sen} \bar{\theta}\right)\right\} \\
=k \int_{\bar{\theta}}^{\theta} F_{1}\left(x_{0}, y_{0}, \cos \alpha, \operatorname{sen} \alpha\right) \operatorname{sen}(\theta-\alpha) d \alpha ;
\end{gathered}
$$

ed essendo sempre $F_{1}\left(x_{0}, y_{0}, x^{\prime}, y^{\prime}\right) \geqslant 0$ e $F_{1}\left(x_{0}, y_{0}, \bar{x}^{\prime}, \bar{y}^{\prime}\right)>0$, l'integrale che quì figura risulta sempre maggiore di zero, per ogni $\theta$ dell'intervallo $(\bar{\theta}-\pi, \bar{\theta}+\pi)$, escluso il valore $\theta=\bar{\theta}$, cioè per tutti i punti della nostra superficie conica, eccettuati quelli che si trovano sulla generatrice detta.

Consideriamo allora il piano rappresentato dall'equazione

$$
z=x^{\prime}(\mathrm{I}-\lambda) F_{x^{\prime}}\left(x_{0}, y_{0}, \overline{x^{\prime}}, \overline{y^{\prime}}\right)+y^{\prime}(\mathrm{I}-\lambda) F_{y^{\prime}}\left(x_{0}, y_{0}, \overline{x^{\prime}}, \overline{y^{\prime}}\right) \text {. }
$$

Esso passa per l'intersezione del piano tangente sopra indicato e del piano $z=0$, e se $\lambda$ è sufficientemente piccolo e positivo o negativo a seconda che $F\left(x_{0}, y_{0}, \overline{x^{\prime}}, \overline{y^{\prime}}\right)>0$ oppure $<0$, risulta soddisfatta la disuguaglianza

$$
F\left(x_{0}, y_{0}, x^{\prime}, y^{\prime}\right)-\left\{x^{\prime}(\mathrm{I}-\lambda) F_{x^{\prime}}\left(x_{0}, y_{0}, \bar{x}^{\prime}, \bar{y}^{\prime}\right)+y^{\prime}(\mathrm{I}-\lambda) F_{y^{\prime}}\left(x_{0}, y_{0}, \bar{x}^{\prime}, \bar{y}^{\prime}\right)\right\}>0
$$

per tutte le coppie $\left(x^{\prime}, y^{\prime}\right)$ di numeri non ambedue nulli.

Se dunque prendiamo, in (II),

$$
\begin{aligned}
& p_{0}=-(\mathrm{I}-\lambda) F_{x^{\prime}}\left(x_{0}, y_{0}, \bar{x}^{\prime}, \overline{y^{\prime}}\right) \\
& q_{0}=-(\mathrm{I}-\lambda) F_{y^{\prime}}\left(x_{0}, y_{0}, \bar{x}^{\prime}, \overline{y^{\prime}}\right),
\end{aligned}
$$

con $\lambda$ scelto come si è detto, è evidente che, per la continuitè della funzione $F\left(x, y, x^{\prime}, y^{\prime}\right)$ e per la sua omogeneità rispetto a $\left(x^{\prime}, y^{\prime}\right)$, in un cerchio del piano $(x, y)$ di centro $P_{o}$ e raggio $r_{o}$, convenientemente piccolo, è sempre, per tutte le possibili coppie di numeri $x^{\prime}, y^{\prime}$, non nulli insieme,

$$
\bar{F}>\text { o. }
$$

Essendo poi $\bar{F}_{1} \equiv F_{1}$, è anche, contemporaneamente

$$
\bar{F}_{x} \geq 0 \text {. }
$$

Poichè la scelta della coppia $\left(\overline{x^{\prime}}, \overline{y^{\prime}}\right)$ puó essere fatta in infiniti modi, converremo nel seguito, di scegliere per $\left(\overline{x^{\prime}}, \bar{y}^{\prime}\right)$ il punto del cerchio $x^{\prime 2}+y^{\prime 2}=\mathrm{I}$ in cui $F_{\mathrm{I}}\left(x_{0}, y_{0}, x^{\prime}, y^{\prime}\right)$ 
è massima, e qualora di tali punti ve ne fossero diversi, di prendere fra essi quello per il quale l'angolo $\theta$, compreso fra o e $2 \pi$ ( $2 \pi$ escluso) e determinato dalle uguaglianze $\cos \theta=x^{\prime}, \operatorname{sen} \theta=y^{\prime}$, è minimo.

Fissata cosi la coppia $\left(\bar{x}^{\prime}, \bar{y}^{\prime}\right)$ e considerati tutti i $\lambda$ che hanno il segno gid indicato, che sono in modulo $\leqslant$ I e per i quali la disuguaglianza (12) è sempre soddisfatta per tutti gli $x^{\prime}, y^{\prime}$ ammessi, sceglieremo fra essi quello il cui valore assoluto sia uguale alla metà del limite superiore dei valori assoluti dei $\lambda$ stessi. Ciò fatto, prenderemo $r_{0}$ uguale alla metà del limite superiore di tutti $\mathrm{i}$ raggi dei cerchi del piano $(x, y)$ aventi tutti il centro in $P_{\mathrm{o}}$ e internamente ai quali ${ }^{{ }^{17}}$ ) è sempre soddisfatta la disuguaglianza $\bar{F}>0$ per tutte le coppie $\left(x^{\prime}, y^{\prime}\right)$ di numeri non ambedue nulli.

12. Possiamo ora procedere senz'altro alla dimostrazione della proposizione enunciata al principio del $\mathrm{n}^{\circ}$ precedente.

Sia $C_{0}$ una delle curve del $\mathrm{n}^{\circ} 3 \mathrm{e}$, considerato un suo punto qualsiasi $P_{0}$, indichiamo con $\alpha_{0}$ il massimo arco della $C_{\mathrm{o}}$ che contiene il punto $P_{\mathrm{o}}$ e che risulta costituito tutto di punti appartenenti al cerchio $\left(P_{o}, r_{0}\right)$, in cui $r_{0}$ è il raggio determinato al $\mathrm{n}^{\circ}$ precedente. La funzione $\bar{F}$, più sopra determinata, soddisfa alle disuguaglianze $\bar{F}>0$, $\bar{F}_{1} \geqslant 0$ in tutta la parte del campo $A$ interna al cerchio $\left(P_{0}, 2 r_{0}\right)$ e per. tutte le possibile coppie di numeri $x^{\prime}, y^{\prime}$ non ambedue nulli.

Pertanto, in forza della proposizione del $\mathrm{n}^{\circ} \mathrm{ro}$, il suo integrale è sull'arco $\alpha_{0}$ una funzione semicontinua inferiormente, e, preso un $\varepsilon>0$ ad arbitrio, è possibile determinare un numero $\rho_{0}$ maggiore di zero e minore di $r_{0}$ e tale che, per ogni arco $\alpha$ di curva $\left(n^{0} 3\right)$ appartenente ordinatamente all'intorno $\left(\rho_{0}\right)$ di $\alpha_{0}$, sia

$$
\int_{\alpha} \bar{F} d \sigma>\int_{\alpha_{0}} \bar{F} d s-\varepsilon
$$

dove $\sigma$ e $s$ rappresentano le misure degli archi parziali di $\alpha, \alpha_{0}$, rispettivamente.

Ricordando la definizione della $\bar{F}$ (I I), possiamo scrivere, come conseguenza di questa disuguaglianza,

$$
\int_{\alpha} F d \sigma>\int_{\alpha_{0}} F d s-\varepsilon+\left(\int_{\alpha_{0}}\left(p_{0} x^{\prime}+q_{c} y^{\prime}\right) d s-\int_{\alpha}\left(p_{0} x^{\prime}+q_{o} y^{\prime}\right) d \sigma\right) .
$$

La differenza nella parentesi che qui figura è uguale, essendo $p_{0}$ e $q_{0}$ due costanti, a

$$
\int_{p_{\mathrm{o}}^{(\mathrm{o})} P^{(0)}}\left(p_{\mathrm{o}} x^{\prime}+q_{\mathrm{o}} y^{\prime}\right) d s+\int_{p^{(\mathrm{I})} p_{\mathrm{o}}^{(\mathrm{I})}}\left(p_{\mathrm{o}} x^{\prime}+q_{\mathrm{o}} y^{\prime}\right) d s
$$

17) Naturalmente non si terrà nessun conto di quei punti che eventualmente non appartenessero al campo $A$. 
dove $P_{\mathrm{o}}^{(\mathrm{o})}, P_{\mathrm{o}}^{(\mathrm{r})}$ indicano $\mathrm{i}$ due estremi di $\alpha_{0}, P^{(\mathrm{o})}, P^{(\mathrm{r})}$ quelli corrispondenti di $\alpha, P_{\mathrm{o}}^{(\mathrm{o})} P^{(\mathrm{o})}$ il segmento rettilineo che ha per primo estremo $P_{0}^{(0)}$ e per secondo $P^{(0)}$, e analogamente $P^{(\mathrm{x})} P_{\mathrm{o}}^{(\mathrm{x})}$.

La somma sopra scritta tende a zero con $\rho_{0}$ e perciò, se questo numero lo si prende sufficientemente piccolo, 'si ha

$$
\int_{\alpha} F d \sigma>\int_{\alpha_{0}} F d s-2 \varepsilon
$$

la quale disuguaglianza prova che sull'arco $\alpha_{0}$ è funzione semicontinua inferiormente anche l'integrale della $F$.

Possiamo dunque affermare che ogni punto $P_{\mathrm{o}}$ della curva $C_{\mathrm{o}}$ appartiene (come punto interno, fatta eccezione per gli estremi della curva) ad un arco $\alpha_{0}$ della curva stessa, sul quale l'integrale della $F$ e funzione semicontinua inferiormente.

Per il ben noto teorema di Borel-LebesGue ${ }^{18}$ ), fra gli archi $\alpha_{0}$ corrispondenti a tutti i punti della $C_{\mathrm{o}}$, è possibile di sceglierne un numero finito $\alpha_{0}^{(\mathrm{r})}, \alpha_{0}^{(2)}, \ldots, \alpha_{0}^{(n)}$, in modo che ogni punto della curva appartenga ad almeno uno di essi. E sopprimendo in ciascuno di questi archi quelle parti che risultassero ricoprire archi dello stesso gruppo di indice inferiore, si verrà ad ottenere una divisione della curva $C_{0}$ in parti consecutive $\beta_{0}^{(1)}, \beta_{0}^{(2)}, \ldots, \beta_{0}^{(m)}$, su ciascuna delle quali lintegrale della $F$ sard una funzione semicontinua inferiormente.

Se allora si considera una qualunque curva $C\left(\mathrm{n}^{\circ} 3\right)$ appartenente ordinatamente ad un intorno $(p)$ della $C_{o}$, e scelta una delle corrispondenze biunivoche, ordinate $\mathrm{e}$ continue intercedenti fra la $C_{o}$ e la $C$ in modo che la distanza fra i punti corrispondenti risulti sempre minore di $\rho$, si indicano con $\beta^{(1)}, \beta^{(2)}, \ldots, \beta^{(m)}$ gli archi della $C$ che in essa corrispondono ai $\beta_{0}^{(1)}, \beta_{0}^{(2)}, \ldots, \beta_{0}^{(m)}$, si ha che, per $\rho$ sufficientemente piccolo, è

e quindi

$$
\int_{\beta(r)} F d \sigma>\int_{\beta_{0}^{(r)}} F d s-\frac{\varepsilon}{m} \quad(r=1,2, \ldots, m),
$$

$$
\int_{C} F d \sigma>\int_{C_{\mathrm{o}}} F d s-\varepsilon
$$

Ciò dimostra la semicontinuità dell'integrale della $F$ e perciò la proposizione enunciata al principio del $\mathrm{n}^{\circ}$ precedente.

13. Il caso regolare.

Un caso particolare della proposizione or ora provata-è il seguente:

${ }^{18}$ ) Cfr. Ch. De la Vallée Poussin, Integrales de Lebesgue. Fonctions d'ensemble. Classes de BaIRE (Paris, Gauthier-Villars, I916); pp. I3-I4. 
L'integrale regolare è una funzione semicontinua della curva d'integrazione e precisamente, semicontinua inferiormente se è sempre $F_{\mathrm{s}}>\mathrm{o}$, superiormente se $F_{\mathrm{I}}<\mathrm{o}$.

14. Nell'enunciato del $\mathrm{n}^{\circ}$ I I si è escluso che in qualche punto del campo $A$ la $F_{1}\left(x, y, x^{\prime}, y^{\prime}\right)$ possa essere identicamente nulla per tutte le possibili coppie $\left(x^{\prime}, y^{\prime}\right)$. Dobbiamo ora esaminare il caso in cui effettivamente si presentano i punti indicati, che chiameremo eccezionali.

Dimostriamo in primo luogo che:

Se, nel campo $A$ e per tutte le coppie $\left(x^{\prime} y^{\prime}\right)$, è $F_{x} \supseteq 0$ e se per ogni punto eccezionale [nel quale è $F_{\mathrm{r}} \equiv$ o per tutte le coppie $\left.\left(x^{\prime} y^{\prime}\right)\right]$ esiste un intorno in cui la funzione $F$ soddisfa sempre alla disuguaglianza $F \gg 0$, l'integrale della $F$ è una funzione semicontinua inferiormente.

Cominciamo con l'osservare che se $(x, y)$ è un punto eccezionale di $A$, è

$$
F\left(\bar{x}, \bar{y}, x^{\prime}, y^{\prime}\right) \equiv p x^{\prime}+q y^{\prime}
$$

con $p$ e $q$ costanti. Ed infatti, essendo

$$
F_{\mathrm{r}} \equiv \frac{F_{x^{\prime} x^{\prime}}}{y^{\prime 2}} \equiv-\frac{F_{x^{\prime} y^{\prime}}}{x^{\prime} y^{\prime}} \equiv \frac{F_{y^{\prime} y^{\prime}}}{x^{\prime 2}},
$$

dalla $F_{\mathrm{s}}\left(\bar{x}, \bar{y}, x^{\prime}, y^{\prime}\right) \equiv \mathrm{o}$ scende $F_{x^{\prime} x^{\prime}} \equiv F_{x^{\prime} y^{\prime}} \equiv F_{y^{\prime} y^{\prime}} \equiv 0$, in $(\bar{x}, \bar{y})$, vale a dire la $F$, nel punto detto è funzione lineare nel complesso delle variabili $x^{\prime}, y^{\prime}$. Segue perciò, dallipotesi fatta sul segno della $F$ in un intorno di $(\bar{x}, \bar{y})$, che è necessariamente

$$
F\left(\bar{x}, \bar{y}, x^{\prime}, y^{\prime}\right) \equiv \mathrm{o}
$$

perchè, se $p$ e $q$ non fossero entrambi nulli, la $F\left(\bar{x}, \bar{y}, x, y^{\prime}\right)$ potrebbe assumere valori positivi e negativi.

Premesso ciò, consideriamo una qualsiasi curva $C_{o}\left(n^{\circ} 3\right)$ e indichiamo con $E$ l'insieme dei suoi punti eccezionali. Dalla continuita della $F_{1}$ segue subito che l'insieme $E$ è chiuso, Perciò, se ad ogni punto di $E$ facciamo corrispondere il limite superiore $r$ dei raggi dei cerchi che hanno il centro nel punto stesso e dentro i quali resta sempre verificata la $F \geqslant$ o, questi $r$ ammettono su $E$ un minimo non nullo, vale a dire, esiste un $r_{0}>0$ tale che sia sempre $r \geqslant r_{0}$. Di più, sempre per essere $E$ cbiuso, possiamo determinare su $C_{0}$ un numero finito di archi $\alpha_{1}, \alpha_{2}, \ldots, \alpha_{n}$, senza punti comuni, tali da rinchiudere tutti $\mathrm{i}$ punti di $E$ e da avere una lunghezza complessiva, che indicheremo con $\sum_{s=1}^{n} \alpha_{s}$, non superiore a $m(E)+\varepsilon$, dove $m(E)$ rappresenta la misura dell'insieme $E$ (misura contata sulla curva) ed $\varepsilon$ è un numero positivo prefissato ad arbitrio, ma $<\frac{r_{0}}{2}$. 
Abbiamo allora, se $M$ indica il massimo modulo di $F\left(x, y, x^{\prime}, y^{\prime}\right)$ sulla $C_{0}$, quando si prenda come parametro l'arco della curva, e per essere, su $E, F \equiv \mathrm{o}$,

$$
\left|\sum_{s=1}^{n} \int_{\alpha_{s}} F d s\right|<M \varepsilon
$$

Abbiamo inoltre che $\mathrm{i}$ punti degli archi $\alpha$ non appartenenti ad $E$ distano da qualche punto di $E$ di non più di $\varepsilon<\frac{r_{0}}{2}$ e che, percio, tutti $\mathrm{i}$ punti degli $\alpha$ sono centri di cerchi di raggio $\frac{r_{0}}{2}$ nell'interno dei quali è sempre $F \supseteq 0$.

Siano ora $\beta_{1}, \beta_{2}, \ldots, \beta_{m}$ gli archi della $C_{o}$ che si ottengono da essa sopprimendo i punti interni agli $\alpha$. In ciascuno degli archi $\beta$ non è mai $F_{\mathrm{r}} \equiv \mathrm{o}$, in nessun punto, per tutte le possibile coppie $\left(x^{\prime}, y^{\prime}\right)$; possiamo quindi determinare un numero $r_{\mathrm{I}}>0$ in modo che cosi non sia neppure nei punti che distano da quelli degli archi $\beta$ per meno di $r_{1}$. Sopra questi archi $\beta$ l'integrale della $F$ è pertanto, per la proposizione del $\mathrm{n}^{\circ}$ I I, una funzione semicontinua inferiormente, e possiamo determinare un numero positivo $\rho$ in guisa che, se $\beta_{s}^{\prime}$ è un qualsiasi arco di curva $\left(n^{\circ} 3\right)$ appartenente ordinatamente all'intorno $(\rho)$ di $\beta_{s}$, si abbia

$$
\int_{\beta_{s}^{\prime}} F d \sigma>\int_{\beta_{s}} F d s-\frac{\varepsilon}{m} .
$$

Se dunque indichiamo con $\rho_{0}$ un numero positivo minore di $\frac{r_{0}}{2}$ e di $\rho$, e consideriamo una qualsiasi curva $C^{\prime}\left(n^{\circ} 3\right)$ appartenente ordinatamente all'intorno $\left(\rho_{0}\right)$ della $C_{o}$, abbiamo, dopo aver decomposto la $C^{\prime}$ negli archi $\alpha_{s}^{\prime}$ e $\beta_{s}^{\prime}$ corrispondenti agli $\alpha_{s}$ e $\beta_{s}$ secondo una qualunque delle corrispondenze che fra essa curva e la $C_{o}$ si possono porre secondo la definizione di curva appartenente ordinatamente allintorno $\left(\rho_{0}\right)$ della $C_{o}\left(\mathrm{n}^{\circ} 4\right)$,

$$
\begin{aligned}
\int_{C^{\prime}} F d \sigma & =\sum_{\mathrm{i}}^{n} \int_{\alpha_{s}^{\prime}} F d \sigma+\sum_{\mathrm{i}}^{m} \int_{\beta_{s}^{\prime}} F d \sigma \\
& >\sum_{\mathrm{r}}^{m} \int_{\beta_{s}} F d s-\varepsilon
\end{aligned}
$$

perchè sugli archi $\alpha_{s}^{\prime}$ è sempre $F \supseteq 0$. Essendo poi, come gid abbiamo notato,

abbiamo anche

$$
\left|\sum_{r}^{n} \int_{a_{s}} F d s\right|<M \varepsilon
$$

$$
\int_{C^{\prime}} F d \sigma>\sum_{s}^{m} \int_{\beta_{s}} F d s+\sum_{\Gamma}^{n} \int_{a_{s}} F d s-\varepsilon(\mathrm{I}+M),
$$


ossia

$$
\int_{C^{\prime}} F d \sigma>\int_{C_{0}} F d s-\varepsilon(I+M) .
$$

Siccome $M$ è indipendente da $\varepsilon$ e questo $\varepsilon$ può essere preso piccolo a piacere, la disuguaglianza scritta prova la semicontinuità dell'integrale della $F^{{ }^{19}}$ ).

I5. Un caso particolare notevole della proposizione precedente si ha quando in tutto il campo $A$ e per tutte le coppie $\left(x^{\prime}, y^{\prime}\right)$ è sempre

$$
F_{1} \supseteq 0, \quad F \gg 0
$$

Più particolarmente ancora l'integrale $I_{C}$ è funzione semicontinua inferiormente quando sia

$$
F\left(x, y, x^{\prime}, y^{\prime}\right)=g(x, y) \sqrt{x^{\prime 2}+y^{\prime 2}}
$$

con $g(x, y)$ funzione continua e sempre $\supseteq 0$ in $A$; ed anche quando sia

$$
F\left(x, y, x^{\prime}, y^{\prime}\right)=g(x, y) G\left(x, y, x^{\prime}, y^{\prime}\right)
$$

con $g(x, y)$ funxione continua e sempre $\gg 0$ in $A$, e $G\left(x, y, x^{\prime}, y^{\prime}\right)$ funzione soddisfacente alle condizioni ammesse per la $F$ al $\mathrm{n}^{\circ} 2$ ed alle disuguaglianze

pure in tutto $A^{\text {ao }}$ ).

$$
G_{1} \supseteq 0, \quad G \supseteq 0,
$$

16. Proseguendo nell'analisi del caso in cui possono esistere punti eccezionali, supponiamo che siano eccezionali tutti i punti del campo $A$, vale a dire che sia sempre, in tutto il campo $A$ e per tutte le possibili coppie $\left(x^{\prime}, y^{\prime}\right), F_{\mathrm{x}} \equiv$ o. Ciò equivale a supporre che sia sempre

$$
F\left(x, y, x^{\prime}, y^{\prime}\right) \equiv x^{\prime} P(x, y)+y^{\prime} Q(x, y) .
$$

Ammetteremo quì che le funzioni $P\left(x, y_{i}\right), Q(x, y)$ siano definite e continue insieme

19) Un esempio in cui le condizioni del teorema ora dimostrato si trovano verificate è fornito dalla funzione

$$
F\left(x, y, x^{\prime}, y^{\prime}\right)=x^{2}(\mathrm{I}-x)^{2} \sqrt{x^{\prime 2}+y^{\prime 2}}+8 x^{3}(\mathrm{I}-x)^{3} y^{\prime} .
$$

Quì è sempre $F_{1} \geqslant 0$, con $F_{1} \equiv 0$ per $x=0$ e per $x=\mathrm{r}$; e se $\delta$ è positivo e sufficientemente piccolo, e $F \supseteq 0$ per tutti gli $x$ soddisfacenti alla $|x|<\delta$ oppure alla $|1-x|<\delta$. E poi chiaro che non è sempre $F \supseteq 0$, perchè è $F\left(\frac{1}{2}, y, 0,-1\right)=-\frac{1}{16}$.

so) Con $G_{1}$ indichiamo l'invariante di WeIerstrass relativo alla funzione $G$ :

$$
G_{\mathrm{I}} \equiv \frac{G_{x^{\prime} x^{\prime}}}{y^{\prime 2}} \equiv-\frac{G_{x^{\prime} y^{\prime}}}{x^{\prime} y^{\prime}} \equiv \frac{G_{y^{\prime} y^{\prime}}}{x^{12}}
$$


con le due derivate parziali $\frac{\partial P}{\partial y}, \frac{\partial Q}{\partial x}$ in tutto il campo $A$, e che in tutto questo campo risulti soddisfatta l'uguaglianza

$$
\frac{\partial P}{\partial y}=\frac{\partial Q}{\partial x}
$$

Sotto queste condizioni, dimostriamo che l'integrale

$$
\int_{C}\left\{x^{\prime} P(x, y)+y^{\prime} Q(x, y)\right\} d s
$$

è una funzione continua su ogni curva $C_{\circ}\left(\mathrm{n}^{\circ} 3\right)$ completamente interna al campo $A$, intendendo con ció che ogni punto della $C_{\text {o }}$ sia interno al campo $A$.

Sia adunque $C_{0}$ una curva completamente interna ad $A$ e indichiamo con $r_{0}$ un numero positivo convenientemente piccolo cosi che risultino interni ad $A$ tutti $\mathrm{i}$ punti appartenenti a cerchi di raggio $r_{0}$ aventi il centro sulla $C_{0}$.

Sia poi $C$ un'altra curva qualsiasi $\left(n^{\circ} 3\right)$ appartenente ordinatamente ad un intorno $(\rho)$ della $C_{o}$, dove supponiamo $\rho<r_{o}$. Indicati con $P_{o}^{(0)}$ e $P_{o}^{(\mathrm{s})}$ gli estremi della $C_{0}$ (sovrapposti, su $C_{0}$ è chiusa) e con $P^{(0)}, P^{(1)}$ quelli della $C$, e considerati i segmenti rettilinei $P_{o}^{(0)} P^{(0)}, P^{(1)} P_{o}^{(\mathrm{r})}$, segue, per il teorema di GreEN, dalla (13)

$$
\left.\int_{P_{0}^{(0)} P^{(0)}}\left(x^{\prime} P+y^{\prime} Q\right) d s+\int_{C} \cdots+\int_{P^{(x)} P_{0}^{(1)}} \cdots=\int_{C_{0}} \cdots{ }^{21}\right) .
$$

E poichè gli integrali estesi ai segmenti $P_{0}^{(o)} P^{(\mathrm{o})}, P^{(\mathrm{r})} P_{0}^{(\mathrm{r})}$ tendono a zero con $\rho$, preso ad arbitrio un $\varepsilon>0$, si puó determinare $\rho$ in modo che sia sempre

$$
\left|\int_{P_{0}^{(0)} p^{(0)}} \cdots+\int_{P^{(1)} p_{0}^{(1)}} \cdots\right|<\varepsilon
$$

comunque si prendano $P^{(o)}$ e $P^{(1)}$ nei cerchi $\left(P_{o}^{(o)}, p\right),\left(P_{o}^{(x)}, p\right)$, rispettivamente.

Si ha allora

$$
\left|\int_{C}\left\{x^{\prime} P+y^{\prime} Q\right\} d \sigma-\int_{C_{o}}\left\{x^{\prime} P+y^{\prime} Q\right\} d s\right|<\varepsilon,
$$

disuguaglianza che prova la nostra proposizione.

2I) Dalla (I3) scende, per il teorema di GREEN, che l'integrale dell'espressione $x^{\prime} P+y^{\prime} Q$ esteso ad una curva chiusa è sempre uguale a zero. Per altro questa proprietà viene ad essere stabilita solo per quelle curve che si considerano nella dimostrazione del teorema ricordato e che di solito sono le curve semplici. Per applicare la proprietà in questione al caso nostro, basta peró riguardare la curva che consideriamo come limite di una successione di poligonali chiuse, per ciascuna delle quali la proprietà stessa è valida. 
17. Circa le condizioni imposte alle funzioni $P$ e $Q$ nel $n^{\circ}$ precedente, vogliamo osservare che, per la validità del teorema ivi dimostrato, esse possono essere sostituite da queste altre più generali [sufficienti per la validità del teorema di GrEEN ${ }^{22}$ )] e che supporremo verificate in ogni punto interno ad $A$, ad eccezione dell'ultima:

$\left.I^{\circ}\right)$ Le funzioni $P$ e $Q$ siano continue.

$2^{\circ}$ ) La $P$ sia funzione assolutamente continua di $y$ e la $Q$ funzione assolutamente continua di $x$.

$\left.3^{\circ}\right)$ Le derivate parziali $\frac{\partial P}{\partial y}, \frac{\partial Q}{\partial x}$, considerate là dove esistono, siano superficialmente integrabili.

$4^{\circ}$ ) La uguaglianza (13) sia verificata quasi ovunque nell'interno di $A$.

Osserviamo, inoltre, che la uguaglianza (13), supposta verificata in ogni punto interno ad $A$, è sufficiente per la validità del teorema del $\mathrm{n}^{\circ}$ precedente anche sotto la sola condizione che le funzioni $P$ e $Q$ siano differenziabili ${ }^{23}$ ).

Ed infatti, sotto questa ipotesi, l'integrale dell'espressione $x^{\prime} P+y^{\prime} Q$, esteso ad una curva rettificabile chiusa è sempre nullo ${ }^{24}$ ).

I8. La continuita dell'integrale (I4), stabilita al $n^{\circ}$ I6 per le curve completamente interne al campo $A$, puó essere estesa a tutte le curve che appartengono al campo, sotto determinate condizioni relative alle funzioni $P$ e $Q$ oppure relative al campo stesso. Più precisamente si puó affermare che :

l'integrale (I4) è una funzione continua su ogni curva $\mathrm{C}\left(\mathrm{n}^{\circ} 3\right)$ :

a) se le funzioni $P$ e $Q$ possono definirsi in un nuovo campo $A^{\prime}$, tale che ogni punto di $A$ risulti interno ad esso, e in modo che in $A^{\prime}$ le due funzioni godano delle stesse proprietà ammesse al $\mathrm{n}^{\circ} \mathrm{r} 6$ per il campo $A$, e coincidano in quest'ultimo campo con le funzioni date;

b) oppure se, ammesse le condizioni del $\mathrm{n}^{\circ} \mathrm{I} 6$, la frontiera di $A$ è tale che:

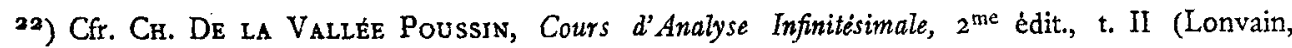
A. Uystprayrt, Dieudonné, 1912); p. \25.

23) Intendiamo che una funzione $₹=f(x, y)$ sia differenziabile in un punto $(x, y)$ se, in tutto un intorno di tal punto, si puo scrivere

$$
f(x+b, y+k)-f(x, y)=A b+B k+\varepsilon
$$

con $A$ e $B$ indipendenti da $b$ e $k$ e $\varepsilon$ infinitesimo di ordine superiore rispetto a $\sqrt{b^{2}+k^{2}}$. Ricordiamo che per la differenziabilità in un punto é condizione sufficiente l'esistenza in esso di una delle due derivate parziali prime e l'esistenza dell'altra in tutto un intorno del punto e la sua continuità nel punto stesso.

24) Cfr. A. Pringsheim, Der Cauchy-Goursatsche Integrulsatz und seine Übertragung auf reelle Kurven-Integrale (Sitzungsberichte der mathem.-phys. Klasse der Kgl. Bayer. Akademie der Wissenschaften, Bd. XXXIII, 1903, pp. 673-682). 
$\mathrm{I}^{\circ}$ ) preso un numero $\rho>0$ qualsiasi ed un punto $M$ pure qualsiasi di $A$, si possa sempre determinare un cerchio di centro $M$ in modo che ogni suo punto appartenente ad $A$ possa congiungersi con $M$ mediante una curva continua, di $A$, di lunghezza $<p$; $2^{\circ}$ ) si possa determinare un numero positivo $R$ in maniera che ogni poligonale chiusa, senza punti multipli, di $A$, avente la massima corda minore di $R$, non racchiuda che punti di $A ; 3^{\circ}$ ) considerata una qualunque curva continua e rettificabile di $A$ e fissato comunque un suo intorno, si possa sempre trovare una poligonale ordinatamente appartenente a questo intorno avente lunghezza diversa da quella della curva di quanto poco si vuole, e tutta costituita di punti interni ad $A$.

Il caso $b$ ) si verifica certamente se la parte della frontiera di $A$ contenuta in ogni porzione limitata di piano è costituita da una curva convessa, oppure da una poligonale, ecc. Quando $P$ e $Q$ sono costanti è poi verificato sicuramente il caso $a$ ).

I9. 'Ritornando ora al caso generale dell'integrale

$$
I_{C}=\int_{C} F d s,
$$

possiamo dedurre dalle precedenti considerazioni che:

Se, in ogni punto del campo $A$, è sempre $F_{\mathrm{r}} \supseteq 0$ ed è possibile di determinare due funzioni $P(x y), Q(x y)$, continue insieme con le due derivate parziali $\frac{\partial P}{\partial y}, \frac{\partial Q}{\partial x}$ in tutto $A$ e in esso sempre soddisfacenti alla uguaglianza

$$
\frac{\partial P}{\partial y}=\frac{\partial Q}{\partial x},
$$

e inoltre tali che nello stesso campo sia sempre

$$
F\left(x, y, x^{\prime}, y^{\prime}\right)+\left\{x^{\prime} P(x y)+y^{\prime} Q(x y)\right\} \geqq 0,
$$

l'integrale $I_{C}$ risulta funzione semicontinua inferiormente su ogni curva $\left(n^{\circ} 3\right)$ completamente interna ad $A$; e lo è poi anche su qualsiasi curva appartenente ad $A$ se è verificata una qualsiasi delle due condizioni enunciate al $n^{\circ}{ }_{1} 8$.

Posto infatti

$$
\bar{F} \equiv F+\left\{x^{\prime} P+y^{\prime} Q\right\}
$$

è sempre $\bar{F}_{1} \equiv F_{1} \supseteq 0$ e per la (I5) anche $\bar{F}_{0} \gg$ o. Dal teorema del no Is segue dunque la semicontinuità di $\int_{C} \bar{F} d s$; vale a dire, preso ad arbitrio un $\varepsilon>0$, è possibile di determinare un $\rho>0$ tale che, se $C^{\prime}$ è una qualsiasi curva $\left(n^{\circ} 3\right)$ appartenente ordinatamente all'intorno $(\rho)$ della $C$, si abbia

$$
\int_{C^{\prime}} \bar{F} d \sigma>\int_{C} \bar{F} d s-\varepsilon,
$$

Rend. Circ. Matem. Palermo, t. XLIV (1920). - Stampato il I6 settembre 1920. 
ossia

$$
\int_{C^{\prime}} F d \sigma>\int_{C} F d s-\varepsilon+\left[\int_{C}\left\{x^{\prime} P+y^{\prime} Q\right\} d s-\int_{C^{\prime}}\left\{x^{\prime} P+y^{\prime} Q\right\} d \sigma\right] .
$$

Ma per le condizioni cui soddisfano le funzioni $P$ e $Q$ e per $\mathrm{i}$ teoremi dei $\mathrm{n}^{\mathrm{i}} \mathrm{r} 6$ e I8, l'integrale della $x^{\prime} P+y^{\prime} Q$ è funzione continua della curva d'integrazione su ogni curva completamente interna ad $A$, oppure su qaalunque curva se è soddisfatta anche una delle due ipotesi del $\mathrm{n}^{\circ}$ i 8 . Supposto dunque che $C$ sia completamente interna ad $A$ oppure che sia verificata una delle due condizioni cui si è accennato, la differenza

$$
\int_{C}\left\{x^{\prime} P+y^{\prime} Q\right\} d s-\int_{C^{\prime}}\left\{x^{\prime} P+y^{\prime} Q\right\} d \sigma
$$

per $\rho$ sufficientemente piccolo, si puó rendere minore di $\frac{\varepsilon}{2}$, e risulta così

$$
\int_{C^{\prime}} F d \sigma>\int_{C} F d s-\frac{3}{2} \varepsilon
$$

Ciò prova la nostra proposizione.

20. Il teorema precedente conserva la sua piena validità anche se, ferme restando le altre ipotesi, la determinazione delle funzioni $P(x y), Q(x y)$, nel modo indicato, anzichè in tutto il campo, è possibile soltanto in un intorno, comunque piccolo, di ciascun punto eccezionale, dove cioè è $F_{\mathrm{s}} \equiv \mathrm{o}$ per tutte le possibili coppie $\left(x^{\prime}, y^{\prime}\right)$.

Per assicurarsi di cio basta ragionare come si è fatto al $\mathrm{n}^{\circ}$ r2, osservando che, considerata una curva $C$ e un suo punto $P$, se questo punto è eccezionale, appartiene ad un arco della $C$ su cui l'integrale della $F$ e semicontinuo inferiormente, in forza della stessa proposizione precedente, e se $P$ non è eccezionale l'esistenza di un arco simile è garantita dal teorema del $\mathrm{n}^{\circ}$ i ${ }^{25}$ ).

21. Dalla proposizione del $\mathrm{n}^{0}$ ig e per l'osservazione fatta or ora puó dedursi una

25) Un esempio relativo al caso qui considerato è il seguente:

$$
F=y^{2}\left(I-y^{2}\right)^{2} \sqrt{x^{\prime 2}+y^{\prime 2}}+\frac{I}{10}\left\{\frac{I}{2}-\left(I-y^{2}\right)^{2}\right\}\left\{I+\left(I-y^{2}\right)^{2}\right\} x^{\prime} .
$$

Per questa funzione è sempre

$$
F_{1}=\frac{y^{2}\left(1-y^{2}\right)^{2}}{\left(x^{\prime 2}+y^{\prime 2}\right)^{3 / 2}} \geqslant 0
$$

con $F_{\mathrm{I}} \equiv$ o per $y=$ o e $y= \pm \mathrm{I}$.

Per $\varepsilon$ sufficientemente piccolo, si ha $F+\frac{1}{10} x^{\prime} \geqslant 0 ;$ quando sia $|y|<\varepsilon ; F-\frac{1}{20} x^{\prime} \geqslant 0$, quando $\left|I-y^{2}\right|<\varepsilon$. 
conseguenza notevole relativamente alle funzioni $F$ della forma

$$
F \equiv \varphi(b x+k y) G\left(x, y, x^{\prime}, y^{\prime}\right) \text {. }
$$

Si supponga che :

$\left.I^{\circ}\right) \varphi(u)$ sia una funzione nion negativa, continua insieme con la sua prima derivata, per tutti $i$ valori di $u$ definiti dall'uguaglianza $u=b x+k y$, dove $b$ e $k$ sono due costanti assegnate e $(x y)$ è un punto qualsiasi del campo $A$;

$\left.2^{\circ}\right) G\left(x, y, x^{\prime}, y^{\prime}\right)$ sia una funzione soddisfacente alle condizioni poste al $\mathrm{n}^{\circ} 2$ per la $F$ e alla disuguaglianza $\left.G_{1}\left(x, y, x^{\prime}, y^{\prime}\right) \gg 0^{0}{ }^{26}\right)$ in tutto il campo $A$, senza che in nessun punto di esso sia mai $G_{3} \equiv$ o per tutte le possibile coppie $\left(x^{\prime} y^{\prime}\right)$;

$\left.3^{\circ}\right)$ si abbia, in ogni punto di $A$ dove è $\varphi(b x+k y)=0, G>0$ per quelle coppie $\left(x^{\prime} y^{\prime}\right)$ di numeri non ambedue nulli che verificano l'uguaglianza $b x^{\prime}+k y^{\prime}=0$.

Sotto queste ipotesi, l'integrale

$$
\int_{C} \varphi(b x+k y) G\left(x, y, x^{\prime}, y^{\prime}\right) d s
$$

è una funzione semieontinua inferiormente su ogni curva $\left(\mathrm{n}^{\circ} 3\right)$ appartenente ad $A$.

Per provarlo, cominciamo col dimostrare che, se $\left(x_{0}, y_{0}\right)$ è un punto di $A$ in cui . ̀̀ $\varphi\left(b x_{0}+k y_{0}\right)=0$, è possibile di determinare un numero $p_{0}$ in guisa che si abbia

$$
G\left(x_{0}, y_{0}, x^{\prime}, y^{\prime}\right)+p_{0}\left(b x^{\prime}+k y^{\prime}\right)>0,
$$

per tutte le coppie $\left(x^{\prime}, y^{\prime}\right)$ di numeri non ambedue nulli.

Preso un sistema di assi cartesiani ortogonali $\left(x^{\prime}, y^{\prime}, z\right)$, la superficie conica rappresentata dall'equazione

$$
z=G\left(x_{0}, y_{0}, x^{\prime}, y^{\prime}\right)
$$

resta, per le considerazioni svolte al $n^{0}$ I $\mathrm{I}$, tutta al disopra di un qualunque suo piano tangente e tocca questo piano in tutti $\mathrm{i}$ punti di una sua generatrice o di un'infinita di generatrici riempienti un angolo minore di $2 \pi$. La superficie conica risulta perció convessa, e per la retta $b x^{\prime}+k y^{\prime}=0$ del piano $\left(x^{\prime} y^{\prime}\right)$, la quale in forza della condizione $3^{\mathrm{a}}$ ) risulta esterna alla superficie, si potranno condurre almeno due piani tangenti alla superficie medesima. L'equazione di uno qualunque di questi piani potrà scriversi

$$
z=p\left(b x^{\prime}+k y^{\prime}\right)
$$

con $p$ costante, e si avrd sempre

$$
G\left(x_{0}, y_{0}, x, y^{\prime}\right)-p\left(b x^{\prime}+k y^{\prime}\right) \supseteq 0 .
$$

${ }^{26)}$ Con $G_{\mathrm{I}}$ indichiamo ancora l'invariante di WeIERStrass relativo alla $G$. 
Inoltre, per la proprietà ricordata della retta $b x^{\prime}+k y^{\prime}=0$, le generatrici di contatto della superficie conica e del piano tangente sopra scritto si troveranno, su questo stesso piano, tutte dalla medesima parte della retta detta e potrà scegliersi un numero $\lambda$ sufficientemente piccolo, e positivo o negativo a seconda che sulle generatrici di contatto sard $G>0$ oppure $G<0$, in maniera che si abbia

$$
G\left(x_{0}, y_{0}, x^{\prime}, y^{\prime}\right)-p(\mathrm{I}-\lambda)\left(b x^{\prime}+k y^{\prime}\right)>0 \text {, }
$$

per tutte le possibile coppie $\left(x^{\prime} y^{\prime}\right)$ di numeri non ambedue nulli. Prendendo dunque $p_{0}=-p(\mathrm{I}-\lambda)$ si ha quanto più sopra si è affermato. Ed è evidente che si puó, in molti modi, fissare un criterio per scegliere un valore di $p_{0}$ fra gli infiniti valori che esso ammette.

Per la continuità' e l'omogeneità della funzione $G$, possiamo aggiungere che, in corrispondenza del punto $\left(x_{0}, y_{0}\right)$, è possibile determinare un numero positivo $p_{0}$ cosi che, in tutto il cerchio di centro $\left(x_{0}, y_{0}\right)$ e raggio $p_{0}$, si abbia

$$
G\left(x, y, x^{\prime}, y^{\prime}\right)+p_{0}\left(b x^{\prime}+k y^{\prime}\right)>0 \text {, }
$$

per tutte le solite coppie $\left(x^{\prime} y^{\prime}\right)$ di numeri non ambedue nulli. Nello stesso cerchio $\dot{e}$ allora

$$
\varphi(b x+k y) G\left(x, y, x^{\prime}, y^{\prime}\right)+\varphi(b x+k y) p_{\circ}\left(b x^{\prime}+k y^{\prime}\right) \geqq 0,
$$

e siccome è sempre

$$
\frac{\partial\left\{p_{0} b \varphi(b x+k y)\right\}}{\partial y}=\frac{\partial\left\{p_{0} k \varphi(b x+k y)\right\}}{\partial x},
$$

la semiconținuità dell'integrale (16) segue senz'altro da quanto si è stabilito ai $\mathrm{n}^{\mathrm{i}}$ I9 e 20 .

22. E utile osservare che, se la funzione $G$ è indipendente da $x$ e $y$, alla $3^{\text {a }}$ condizione della proposizione dimostrata ora puó sostituirsi quest'altra, che sia cioè « $G \gg 0$ per quelle coppie $\left(x^{\prime}, y^{\prime}\right)$ di numeri che verificano l'uguaglianza $b x^{\prime}+k y^{\prime}=0$ ». Ed invero, si potra, sotto questa condizione, determinare un numero $p$ tale che si abbia

$$
G\left(x^{\prime}, y^{\prime}\right)+p\left(b x^{\prime}+k y^{\prime}\right) \gg 0,
$$

per tutti i possibili valori di $x^{\prime}, y^{\prime}$, e quindi si potrd soddisfare alle condizioni dell'enunciato del $n^{\circ}$ i $9^{27}$ ).

27) Sia per es. $F=(x+y)^{2}\left(\sqrt{5 x^{\prime 2}+4 y^{\prime 2}}-3 x^{\prime}\right)$ e si consideri un campo $A$ contenente l'origine delle coordinate. E $F\left(0,0, x^{\prime}, y^{\prime}\right) \equiv 0 ; G=\sqrt{5 x^{\prime 2}+4 y^{\prime 2}}-3 x^{\prime}$, e percio $G_{\mathrm{I}}=\frac{4}{\sqrt{5 x^{\prime 2}+4 x^{\prime 2}}}>0$. E inoltre $\varphi(b x+k y)=(x+y)^{2}$, e se è $x^{\prime}+y^{\prime}=0$, è $G=0$ per $x^{\prime} \supseteq 0, G=6\left|x^{\prime}\right|$ per $x^{\prime}<0$. Prendendo qui $p=\frac{4}{3}$, si ha sempre

$$
G+\frac{4}{3}\left(x^{\prime}+y^{\prime}\right)=\sqrt{5 x^{\prime 2}+4 y^{\prime 2}}-\frac{5}{3} x^{\prime}+\frac{4}{3} y^{\prime} \geqslant 0 \text {. }
$$


23. Supponiamo di aver sempre, in ogni punto di $A, F_{1} \gg 0$ e che, in ogni punto eccezionale $\left(x_{0}, y_{0}\right)$ interno al campo, sia verificata la

$$
F_{x^{\prime} y}\left(x_{0}, y_{0}, x^{\prime}, y^{\prime}\right) \equiv F_{y^{\prime} x}\left(x_{0}, y_{0}, x^{\prime}, y^{\prime}\right)
$$

per tutte le possibili coppie $\left(x^{\prime}, y^{\prime}\right)$.

Come già si è osservato altrove ( $\mathrm{n}^{0}$ I 4$)$, in ogni punto eccezionale $\left(x_{0}, y_{0}\right)$ deve essere necessariamente

$$
F\left(x_{0}, y_{0}, x^{\prime}, y^{\prime}\right) \equiv p_{0} x^{\prime}+q_{0} y^{\prime}
$$

con $p_{0}$ e $q_{0}$ costanti. Inoltre, per essere sempre $F_{1} \supseteq 0$ e $F_{1}\left(x_{0}, y_{0}, x^{\prime}, y^{\prime}\right) \equiv 0$, il punto $\left(x_{0}, y_{0}\right)$ costituisce un minimo per la $F_{1}$, e quindi anche per le $F_{x^{\prime} x^{\prime}}, F_{y^{\prime} y^{\prime}}$, come risulta dalla definizione stessa della $F_{\mathrm{I}}$

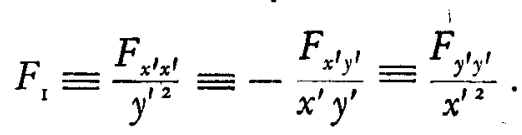

E dunque, per $x=x_{0}, y=y_{0}$,

$$
F_{x^{\prime} x^{\prime} x} \equiv F_{x^{\prime} x^{\prime} y} \equiv F_{y^{\prime} y^{\prime} x} \equiv F_{y^{\prime} y^{\prime} y} \equiv \mathrm{o},
$$

identicamente per tutte le coppie $\left(x^{\prime}, y^{\prime}\right)$. Da ciò segue che $F_{x}\left(x_{0}, y_{0}, x^{\prime}, y^{\prime}\right)$ e $F_{y}\left(x_{0}, y_{0}, x^{\prime}, y^{\prime}\right)$ sono funzioni lineari nella $x^{\prime}$ e pure lineari nella $y^{\prime}$, e sard

$$
\begin{aligned}
& F_{x}\left(x_{0}, y_{0}, x^{\prime}, y^{\prime}\right) \equiv p_{1} x^{\prime}+q_{1} y^{\prime}+r_{1} x^{\prime} y^{\prime}, \\
& F_{y}\left(x_{0}, y_{0}, x^{\prime}, y^{\prime}\right) \equiv p_{2} x^{\prime}+q_{2} y^{\prime}+r_{2} x^{\prime} y^{\prime} .
\end{aligned}
$$

Ma la $F_{\mathrm{I}} \gg \mathrm{o}$ porta che sia sempre $-\frac{F_{x^{\prime} y^{\prime}}}{x^{\prime} y^{\prime}} \supseteq 0$ e quindi $F_{x^{\prime} y^{\prime}}(x, y, 0, \mathrm{r}) \equiv \mathrm{o}$, donde

$$
F_{x^{\prime} y^{\prime} x}(x, y, \text { o, I }) \equiv \mathrm{o}, \quad F_{x^{\prime} y^{\prime} y}(x, y, \text { o, I }) \equiv \mathrm{o} .
$$

$\mathrm{E}$ pertanto $r_{1}=r_{2}=0$, vale a dire

$$
\left\{\begin{array}{l}
F_{x}\left(x_{0}, y_{0}, x^{\prime}, y^{\prime}\right) \equiv p_{\mathrm{I}} x^{\prime}+q_{1} y^{\prime}, \\
F_{y}\left(x_{0}, y_{0}, x^{\prime}, y^{\prime}\right) \equiv p_{2} x^{\prime}+q_{2} y^{\prime} .
\end{array}\right.
$$

Da ciò segue che, in $\left(x_{0}, y_{0}\right)$, le derivate $F_{x x^{\prime}}, F_{x y^{\prime}}, F_{y x^{\prime}}, F_{y y^{\prime}}$, sono costanti rispetto alle $x^{\prime}, y^{\prime}$ e precisamente uguali a $p_{\mathrm{I}}, q_{1}, p_{2}, q_{2}$, rispettivamente.

$\mathrm{La}$ (I7) diventa, percio,

$$
p_{2}=q_{1}
$$

e possiamo rilevare che, per poter affermare la validitd della (17) per tutte le coppie $\left(x^{\prime} y^{\prime}\right)$, basta assicurarsi della validità sua per una sola coppia.

Ciò posto, definiamo, in un intorno del punto $\left(x_{0}, y_{0}\right)$ due funzioni $P(x, y)$, 
$Q(x, y)$, continue insieme con le loro derivate parziali prime, in modo da soddisfare alle condizioni

$$
\begin{gathered}
P\left(x_{0}, y_{0}\right)=p_{0}, \\
\frac{\partial P}{\partial y}\left(x_{0}, y_{0}\right)=p_{2}, \\
\frac{\partial P}{\partial x}\left(x_{0}, y_{0}\right)=p_{1}, \\
Q(x y)=\int_{x_{0}}^{x} \frac{\partial P}{\partial y} d x+\varphi(y), \\
\varphi\left(y_{0}\right)=q_{0}, \\
\varphi^{\prime}\left(y_{0}\right)=q_{2},
\end{gathered}
$$

dalle quali segue che, in tutto l'intorno detto, è

$$
\frac{\partial Q}{\partial x}=\frac{\partial P}{\partial y} \text {. }
$$

Considerata poi la funzione

$$
\bar{F}\left(x, y, x^{\prime}, y^{\prime}\right) \equiv F\left(x, y, x^{\prime}, y^{\prime}\right)-\left\{x^{\prime} P(x, y)+y^{\prime} Q(x, y)\right\},
$$

abbiamo che, nell'intorno di $\left(x_{0}, y_{0}\right)$ in cui essa risulta definita, è sempre soddisfatta la $\bar{F}_{1} \geqslant 0$ ed è $\bar{F}_{1}\left(x_{0}, y_{0}, x^{\prime}, y^{\prime}\right) \equiv 0$; è anche, tenendo conto delle (I8), (I9), (I $\left.7^{\prime}\right)$, (20), (21),

$$
\begin{gathered}
\bar{F}\left(x_{0}, y_{0}, x^{\prime}, y^{\prime}\right) \equiv 0, \\
\bar{F}_{x}\left(x_{0}, y_{0}, x^{\prime}, y^{\prime}\right) \equiv\left\{p_{1} x^{\prime}+q_{1} y^{\prime}\right\}-\left\{p_{1} x^{\prime}+q_{1} y^{\prime}\right\} \equiv 0, \\
\bar{F}_{y}\left(x_{0}, y_{0}, x^{\prime}, y^{\prime}\right) \equiv\left\{p_{2} x^{\prime}+q_{2} y^{\prime}\right\}-\left\{p_{2} x^{\prime}+q_{2} y^{\prime}\right\} \equiv 0 .
\end{gathered}
$$

Se, data l'arbitrarietà che le condizioni (20) consentono alla definizione delle funzioni $P(x y), Q(x y)$, si possono scegliere queste funzioni in modo che, in un cerchio di centro $\left(x_{0}, y_{0}\right)$ e raggio $p_{0}$, convenientemente piccolo [da prendersi cosi piccolo che tutti i punti del cerchio risultino punti interni ad $A$, come lo è, per ipotesi, $\left(x_{0}, y_{0}\right)$ ], la $\bar{F}$ abbia in $\left(x_{0} y_{0}\right)$ un minimo, allora, in tutto il cerchio detto, è

$$
\left.\bar{F} \supseteq 0^{28}\right)
$$

${ }^{28}$ ) Per essere sempre $\bar{F}_{1} \supseteq 0$ si può dire che, comunque si scelgano le $P$ e $Q$, su ogni punto $\overline{(x}, \bar{y})$ di un intorno di $\left(x_{0}, y_{0}\right)$ e $\bar{F} \supseteq 0$ per tutte le coppie $\left(x^{\prime}, y^{\prime}\right)$ di almeno metà del cerchio $x^{\prime 2}+y^{\prime 2}=$ I e per tutte quelle ad esse proporzionali, con fattore di proporzionalità positivo. 
e tale disuguaglianza, unita alla $\bar{F}_{1} \geqslant 0$ e alla (2I) permette di affermare, in forza del teorema del $\mathrm{n}^{0} \mathrm{I} 9$, che su ogni curva contenuta nel medesimo cerchio l'integrale della $F$ è funzione semicontinua inferiormente.

Considerata poi una curva $C$ completamente interna ad $A$, e supposto che quanto si è detto ora si verifichi per ogni punto eccezionale, risulta $\left(\mathrm{n}^{0} 20\right)$ senz'altro la semicontinuità inferiore di $I_{C}$ 'sulla $C$.

24. In ciò che precede, per stabilire la semicontinuitd di $I_{C}$, nel caso quasi-regolare, abbiamo sempre aggiunto alla condizione $F_{1} \supseteq 0$ qualche altra condizione complementare.

Nel $\int 6$ di questo Capitolo si vedrà che la condizione $F_{1} \gg 0$ non è di per sè sufficiente ad assicurare la semicontinuità inferiore dell'integrale della $F$; tuttavia, nel caso generale quasi-regolare si puó dimostrare una proposizione, di notevole importanza nei riguardi del Calcolo delle Variazioni, la quale stabilisce per il nostro integrale una specie di semicontinuitd inferiore limitata. Si può cioè dimostrare che:

Preso ad arbitrio un numero positivo $L$, l'integrale quasi-regolare $\dot{e}$ una funzione semicontinua nella classe delle curve $\left(\mathrm{n}^{\circ} 3\right)$ non superiori in lunghezza al numero $L$.

Scelto comunque un $\varepsilon>0$, prendiamo

e consideriamo la funzione

$$
n=\frac{\varepsilon}{2 L}
$$

$$
\bar{F}\left(x, y, x^{\prime}, y^{\prime}\right) \equiv F\left(x, y, x^{\prime}, y^{\prime}\right)+n \sqrt{x^{\prime 2}+y^{\prime 2}} .
$$

Essa soddisfa in ogni punto di $A$ alla disuguaglianza $\bar{F}_{1} \supseteq 0-$ supposto che sia sempre $F_{1} \supseteq 0-$ per tutte le coppie $\left(x^{\prime} y^{\prime}\right)$ di numeri non ambedue nulli, e quindi, in forza del teorema del $\mathrm{n}^{0} \mathrm{r}_{3}$, fissata una curva $C_{0}\left(\mathrm{n}^{0} 3\right)$ di lunghezza non superiore ad $L$, possiamo determinare un numero $\rho>0$ in modo che sia

$$
\int_{C} \bar{F} d \sigma>\int_{C_{\mathrm{o}}} \bar{F} d s-\frac{\varepsilon}{2}
$$

per tutte le curve $C\left(\mathrm{n}^{\circ} 3\right)$ appartenenti. ordinatamente all'intorno $(\rho)$ della $C_{0}$.

Se ora consideriamo fra le $C$ soltanto quelle curve $C^{\prime}$ che hanno lunghezza non superiore ad $L$, possiamo dedurre dalla disuguaglianza precedente

$$
\int_{C^{\prime}} F \sigma+n L>\int_{C_{\mathrm{o}}} F d s+n \int_{C_{\mathrm{o}}} d s-\frac{\varepsilon}{2},
$$

e per il modo nel quale è stato scelto il numero $n$,

$$
\int_{C^{\prime}} F d \sigma>\int_{C_{\mathrm{o}}} F d s-\varepsilon
$$

Questo basta per provare la proposizione enunciata. 
25. Possiamo enunciare anche il seguente corollario:

Se $P(x y)$ e $Q(x y)$ sono due funzioni continue nel campo $A$, preso ad arbitrio un numero positivo $L$, lintegrale

$$
\int_{C}\left\{x^{\prime} P(x y)+y^{\prime} Q(x y)\right\} d s
$$

è una funzione continua nella classe delle curve $\left(\mathrm{n}^{\circ} 3\right)$ che banno lunghezza non superiore al numero $L$.

\section{$\$ 5$ \\ Condizioni sufficienti per la semicontinuità.}

c) Là semicontinuità su una data curva.

26. Sino ad ora ci siamo sempre occupati di stabilire la semicontinuità di $I_{C}$ su tutte le curve del campo $A$ oppure su tutte quelle completamente interne al campo stesso. Ora dobbiamo portare la nostra attenzione al problema di assegnare le condizioni sufficienti affinchè, data una curva $C$, si possa asserire che sopra di essa l'integrale $I_{C}$ è funzione semicontinua. E ben evidente che tutti i teoremi dei $\int \oint 3$ e 4 dàno senz'altro altrettante proposizioni per il caso attuale, quando si suppongano verificate in tutto un intorno della curva considerata le condizioni che in essi figurano. Alcune di queste proposizioni possono però enunciarsi supponendo le condizioni relative verificate soltanto sulla curva $C$, senza tener alcun conto di quanto avviene nell'intorno della curva medesima. Si ha cosi che:

l'integrale $I_{C} \dot{e}$, sulla curva data $C$, funzione semicontinua inferiormente se, in ogni punto della $C$ e per ogni coppia $\left(x^{\prime} y^{\prime}\right)$ di numeri non ambedue nulli, $\dot{e}$ verificata una delle due condizioni seguenti:

I $\left.^{\circ}\right) F_{1} \supseteq 0, F>0$

$\left.2^{\circ}\right) F_{\mathrm{I}} \supseteq 0$ senza che in nessun punto di $C$ sia $F_{\mathrm{r}}\left(x, y, x^{\prime}, y^{\prime}\right)$ identicamente nulla per tutte le coppie $\left(x^{\prime} y^{\prime}\right)$ ammesse.

Consideriamo il primo caso. Essendo sulla $C$ sempre $F>0$, è possibile per la continuita di determinare un intorno $(p)$ di essa in ogni cui punto appartenente ad $A$ sia sempre $F>$ o per tutte le coppie $\left(x^{\prime} y^{\prime}\right)$ di numeri non ambedue nulli. Sostituendo allora, al campo $A$, quella sua parte che appartiene all'intorno detto della $C$, si puó ripetere interamente la dimostrazione del $n^{\circ}$ Io.

Passando al secondo caso, si ha senz'altro che i ragionamenti dei $n^{i}$ I I e i 2 possono ripetersi con la sola avvertenza di restringere la considerazione della disuguaglianza $F_{\mathrm{t}} \supseteq 0$ ai punti di $C$ e di sfruttare il caso precedente. 
Anche la proposizione del $\mathrm{n}^{\circ} 24$ vale sulla curva $C$ supponendo solo che si abbia sempre su tal curva $F, \gg 0$. Ed infatti, riprendendo la funzione $\bar{F}$ definita al $\mathrm{n}^{\mathbf{o}}$ indicato, la disuguaglianza $\bar{F}_{1} \gg n$, valida su tutta la $C$ quando sia $x^{\prime 2}+n^{\prime 2}=1$, porta l'altra $\widetilde{F}_{1} \supseteq \frac{n}{2}$, valida in tutto un intorno della curva medesima e per le stesse coppie $\left(x^{\prime}, n^{\prime}\right)$.

Per le altre proposizioni dei $\$ \$ 3$ e 4 , si puó affermare che la condizione $F_{1} \geq 0$, che in esse sempre figura, basta supporla verificata solamente sui punti della curva considerata.

27. A quanto precede vogliamo aggiungere un'altra proposizione notevole.

Sia $C_{0}$ una curva $\left(\mathrm{n}^{\circ} 3\right)$ di $A$ e si indichi $\operatorname{con} \theta_{0}$ l'angolo (compreso fra zero e $2 \pi$, secondo estremo escluso) che la direzione positiva della tangente alla curva, là dove essa tangente esiste, forma con quella, pure positiva, dell'asse delle $x$.

Posto

$E\left(x, y ; x^{\prime}, y^{\prime} ; \overline{x^{\prime}}, \overline{y^{\prime}}\right) \equiv F\left(x, y, \overline{x^{\prime}}, \overline{y^{\prime}}\right)-\left\{\overline{x^{\prime}} F_{x^{\prime}}\left(x, y, x^{\prime}, y^{\prime}\right)+\overline{y^{\prime}} F_{y^{\prime}}\left(x, y, x^{\prime}, y^{\prime}\right)\right\}$, si ha:

lintegrale $I_{C}$ è sulla curva $C_{0}$ una funzione semicontinua inferiormente se:

a) in ogni punto $\left(x_{0}, y_{0}\right)$ di $C_{o}$, dove esiste la tangente alla curva, $\dot{e}$

$$
E\left(x_{0}, y_{0} ; \cos \theta_{0}, \operatorname{sen} \theta_{0} ; \cos \bar{\theta}, \operatorname{sen} \bar{\theta}\right)>0
$$

per tutti gli angoli $\bar{\theta}$, compresi fra zero e $2 \pi$, distinti da $\theta_{0}$;

b) per ogni punto $\left(\hat{x}_{\mathrm{o}}, \hat{y}_{\mathrm{o}}\right)$ di $C_{\mathrm{o}}$, dove non esiste la tangente alla curva, esiste un angolo $\vec{\theta}_{0}$, compreso fra zero e $2 \pi$, tale che sia

$$
E\left(\hat{x}_{0}, \bar{y}_{0} ; \cos \hat{\theta}_{0}, \operatorname{sen} \bar{\theta}_{0} ; \cos \bar{\theta}, \operatorname{sen} \bar{\theta}\right)>0
$$

ancora per tutti $i \bar{\theta}$, compresi fra zero e $2 \pi$, distinti da $\bar{\theta}_{0}$.

Sia $\left(\bar{x}_{0}, \bar{y}_{0}\right)$ un qualsiasi punto della curva $C_{0}$. Per le ipotesi fatte, esiste una coppia $\left(\overline{x_{0}^{\prime}}, \overline{y_{0}^{\prime}}\right)$ di numeri non ambedue nulli [uguali rispettivamente a $\cos \theta_{0}, \operatorname{sen} \theta_{0}$, se in $\left(\bar{x}_{0}, \bar{y}_{0}\right)$ esiste la tangente alla curva, a $\cos \bar{\theta}_{0}$, sen $\bar{\theta}_{0}$, in caso contrario] tali da soddisfare alla disuguaglianza.

$$
E\left(\bar{x}_{0}, \bar{y}_{0} ; \bar{x}_{0}^{\prime}, \bar{y}_{0}^{\prime} ; x^{\prime}, \bar{y}^{\prime}\right)>0
$$

per tutte le coppie $\left(\bar{x}^{\prime}, \bar{y}^{\prime}\right)$ di numeri non ambedue nulli e non multipli secondo uno stesso numero positivo di $\bar{x}_{\mathrm{o}}^{\prime}$ e $\bar{y}_{0}^{\prime}$.

Consideriamo, riferita ad un sistema di assi cartesiani ortogonali $x^{\prime}, y^{\prime}, z$, la su- 
perficie conica

$$
z=F\left(\bar{x}_{0}, \bar{y}_{0}, x^{\prime}, y^{\prime}\right)
$$

e il suo piano tangente

$$
z_{t}=x^{\prime} F_{x^{\prime}}\left(\bar{x}_{0}, \bar{y}_{0}, \bar{x}_{0}^{\prime}, \bar{y}_{0}^{\prime}\right)+y^{\prime} F_{y^{\prime}}\left(\bar{x}_{0}, \bar{y}_{0}, \bar{x}_{0}^{\prime}, \bar{y}_{0}^{\prime}\right) \text {. }
$$

Per la disuguaglianza sopra scritta si ha, in tutti i punti $\left(\bar{x}^{\prime}, \bar{y}^{\prime}\right)$ indicati or ora,

$$
z-z_{t}>0
$$

e in tutti gli altri, quelli cioè della generatrice del cono passante per il punto

$$
\begin{gathered}
\left\{x_{0}^{\prime}, y_{0}^{\prime}, z=F\left(\bar{x}_{0}, \bar{y}_{0} ; \bar{x}_{0}^{\prime}, \bar{y}_{0}^{\prime}\right)\right\} \\
z-z_{t}=0 .
\end{gathered}
$$

Ed è evidente che, scegliendo un numero $\lambda$ abbastanza piccolo e positivo o negativo a seconda che sulla generatrice sopra indicata è $z>0$ oppure $z<0$, si avrà, ponendo

$$
\begin{gathered}
\zeta=x^{\prime}(\mathrm{I}-\lambda) F_{x^{\prime}}\left(\bar{x}_{0}, \bar{y}_{0}, \bar{x}_{0}^{\prime}, \bar{y}_{0}^{\prime}\right)+y^{\prime}(\mathrm{I}-\lambda) F_{y^{\prime}}\left(\bar{x}_{0}, \bar{y}_{0}, \bar{x}_{0}^{\prime}, \bar{y}_{0}^{\prime}\right), \\
z-\zeta>0,
\end{gathered}
$$

in tutti i punti $\left(x^{\prime}, y^{\prime}\right)$ ad eccezione dell'origine $(0,0)$. Per la continuita della $F$, si avrà poi soddisfatta la disuguaglianza

$$
F\left(x, y, x^{\prime}, y^{\prime}\right)-\zeta>0
$$

in tutto un intorno del punto $\left(\bar{x}_{0}, \bar{y}_{0}\right)$ e per tutte le coppie $\left(x^{\prime}, y^{\prime}\right)$ di numeri non ambedue nulli.

Indichiamo con $2 \bar{\rho}$ il massimo raggio di un cerchio di centro $\left(\bar{x}_{0}, \bar{y}_{0}\right)$, tutto interno all'intorno detto, e con $\bar{\alpha}$ il massimo arco di $C_{0}$ che contiene $\left(\bar{x}_{0}, \bar{y}_{0}\right)$ e che è contenuto nel cerchio di centro $\left(\bar{x}_{0}, \bar{y}_{0}\right)$ e raggio $\bar{p}$. Posto

$$
\bar{F}\left(x, y, x^{\prime}, y^{\prime}\right) \equiv F\left(x, y, x^{\prime}, y^{\prime}\right)-\zeta
$$

e indicata con $\bar{E}$ la funzione $E$ relativa alla $\bar{F}$, abbiamo [per a) e $b$ )], su tutto l'arco $\bar{\alpha}$,

$$
\widetilde{E}\left(x_{0}, y_{0} ; x_{0}^{\prime}, y_{0}^{\prime} ; \bar{x}^{\prime}, \bar{y}^{\prime}\right)>0
$$

intendendo che $x_{0}^{\prime}, y_{0}^{\prime}$ siano rispettivamente uguali a $\cos \theta_{0}, \operatorname{sen} \theta_{0}$ oppure a $\cos \bar{\theta}_{0}$, $\operatorname{sen} \bar{\theta}_{0}$, a seconda che in $\left(x_{0}, y_{0}\right)$ esiste o no la tangente alla $C_{0}$, e che $\overline{x^{\prime}}, \overline{y^{\prime}}$ siano due qualunque numeri non equimultipli di $x_{0}^{\prime}, y_{0}^{\prime}$ secondo un numero positivo o nullo.

Possiamo allora ripetere per l'arco $\bar{\alpha}$ tutta la dimostrazione del $\mathrm{n}^{0}$ Io, sostituendo 
però alla uguaglianza

l'altra

$$
\begin{gathered}
F\left(x_{0}, y_{0}, x^{\prime}, y^{\prime}\right)=\left\{x^{\prime} F_{x^{\prime}}\left(x_{0}, y_{0}, x_{0}^{\prime}, y_{0}^{\prime}\right)+y^{\prime} F_{y^{\prime}}\left(x_{0}, y_{0}, x_{0}^{\prime}, y_{0}^{\prime}\right)\right\} \\
\quad+\sqrt{x^{\prime 2}+y^{\prime 2}} \int_{\theta_{0}}^{\theta} F_{\mathrm{t}}\left(x_{0}, y_{0}, \cos \alpha \cdot \operatorname{sen} \alpha\right) \operatorname{sen}(\theta-\alpha) d \alpha,
\end{gathered}
$$

$$
\begin{aligned}
\bar{F}\left(x_{0}, y_{0}, x^{\prime}, y^{\prime}\right)= & \left\{x^{\prime} \bar{F}_{x^{\prime}}\left(x_{0}, y_{0}, x_{0}^{\prime}, y_{0}^{\prime}\right)+y^{\prime} \bar{F}_{y^{\prime}}\left(x_{0}, y_{0}, x_{0}^{\prime}, y_{0}^{\prime}\right)\right\} \\
& +\bar{E}\left(x_{0}, y_{0} ; x_{0}^{\prime}, y_{0}^{\prime} ; x^{\prime}, y^{\prime}\right) ;
\end{aligned}
$$

ed osservando che i punti di $\bar{\alpha}$ in cui manca la tangente costituiscono un insieme di misura nulla e quindi completamente trascurabile agli effetti dell'integrazione, e che in quelli ove la tangente esiste vale la disuguaglianza scritta più sopra, relativamente alla $\bar{E}$, per $x_{\mathrm{o}}^{\prime}=\cos \theta_{\mathrm{o}}, y_{\mathrm{o}}^{\prime}=\operatorname{sen} \theta_{\mathrm{o}}$, possiamo scrivere la disuguaglianza

$D_{i}>-\varepsilon\left(s_{i+1}-s_{i}\right)+\int_{s_{i}}^{s_{i+1}}\left\{\left(x^{\prime}-x_{0}^{\prime}\right) F_{x^{\prime}}\left(x_{0} y_{0} x_{0}^{\prime} y_{0}^{\prime}\right)+\left(y^{\prime}-y_{0}^{\prime}\right) F_{y^{\prime}}\left(x_{0} y_{0} x_{0}^{\prime} y_{0}^{\prime}\right)\right\} d s$

e proseguire cosł il ragionamento giungendo a stabilire sull'arco $\bar{\alpha}$ la semicontinuita inferiore dell'integrale di $\bar{F}$ e quindi di quello di $F$, essendo l'integrale della $\zeta$ una funzione continua della linea d'integrazione. Un ragionamento analogo a quello fatto al $\mathrm{n}^{0}$ r 2 ci porta infine a concludere che $I_{C}$ è sulla curva $C_{0}$ una funzione semicontinua inferiormente.

28. Osservazione I. - La proposizione or ora dimostrata continua a sussistere se fra i punti della curva $C_{o}$ in cui esiste la tangente ne esiste un insieme di misura nulla in cui non è verificata la condizione $a$ ), purchè però in essi sia verificata la $b$ ).

Osservazione II. - Se é, sulla $C_{0}$, sempre $F>0$, per tutte le coppie $\left(x^{\prime} y^{\prime}\right)$ di numeri non ambedue nulli, il teorema precedente vale sotto la sola condizione che, eccettuati $i$ punti nei quali non esiste la tangente alla curva e quelli di un qualsiasi insieme di misura nulla, sia soddisfatta la disuguaglianza

$$
E\left(x_{0}, y_{0} ; \cos \theta_{0}, \operatorname{sen} \theta_{0} ; \cos \bar{\theta}, \operatorname{sen} \bar{\theta}\right) \supseteq 0,
$$

per tutti gli angoli $\bar{\theta}_{\text {o }}$.

Osservazione III. - Dalla definizione della funzione $E$ e dalla formula stabilita al $\mathrm{n}^{\circ}$, segue

$E\left(x_{0}, y_{0} ; \cos \theta_{0}, \operatorname{sen} \theta_{0} ; \cos \theta, \operatorname{sen} \theta\right)=\int_{\theta_{0}}^{\theta} F_{\mathrm{r}}\left(x_{0}, y_{0}, \cos \alpha, \operatorname{sen} \alpha\right) \operatorname{sen}(\theta-\alpha) d \alpha$, e applicando il teorema della media, $E\left(x_{0}, y_{0} ; \cos \theta_{0}, \operatorname{sen} \theta_{0} ; \cos \theta, \operatorname{sen} \theta\right)=\left\{I-\cos \left(\theta-\theta_{0}\right)\right\} F_{1}\left(x_{0}, y_{0}, \cos \alpha^{\prime}, \operatorname{sen} \alpha^{\prime}\right)$, con $\alpha^{\prime}=\theta_{0}+t\left(\theta-\theta_{0}\right), 0<t<$ I. La.condizione $\left.a\right)$ porta dunque, nei punti di 
$C_{0}$ ove essa vale,

$$
F_{\mathrm{r}}\left(x_{\mathrm{o}}, y_{\mathrm{o}}, \cos \theta_{\mathrm{o}}, \operatorname{sen} \theta_{\mathrm{o}}\right) \gg 0
$$

e, per le osservazioni precedenti si vede che, pur avendosi sulla $C_{0}$ la semicontinuità inferiore di $I_{C}$, questa disuguaglianza puó non essere soddisfatta in un insieme di punti della curva in cui esiste la tangente, purchè tale insieme sia di misura nulia ed in tutti i suoi punti sia soddisfatta la condizione $b$ ) o purchè tale insieme sia di misura nulla e sia sempre, sulla $C_{0}, F>0$.

Perchè non sorgano dubbi sulla possibilità dell'effettiva esistenza di questo insieme, consideriamo il seguente esempio.

Sia

官

$$
F=\frac{x^{\prime 2}+y^{\prime 2}}{\sqrt{2\left(x^{\prime 2}+y^{\prime 2}\right)-x^{\prime}}} .
$$

$$
\begin{aligned}
& E\left(x, y, \cos \theta, \operatorname{sen} \theta ; \bar{x}^{\prime}, \bar{y}^{\prime}\right)=\frac{\bar{x}^{\prime 2}+\bar{y}^{\prime 2}}{\sqrt{2\left(\bar{x}^{\prime 2}+\bar{y}^{\prime 2}\right)-x^{\prime}}}- \\
& -\frac{\bar{x} \cdot\left\{\sqrt{2} \cos \theta+1-2 \cos ^{2} \theta\right\}+\overline{y^{\prime}} \operatorname{sen} \theta\{\sqrt{2}-2 \cos \theta\}}{(\sqrt{2}-\cos \theta)^{2}} \\
& F_{1}(x, y, \cos \theta, \operatorname{sen} \theta)=\frac{\sqrt{2}(2 \sqrt{2}-3 \cos \theta)}{(\sqrt{2}-\cos \theta)^{3}} \text {. }
\end{aligned}
$$

Scegliamo come curva $C_{\mathrm{o}}$ quella formata nel seguente modo. Sia $a_{1}, a_{2}, \ldots, a_{n}, \ldots$ la successione dei punti del segmento $(O, I)$ definita da:

$$
\begin{aligned}
a_{1} & =\mathrm{I} \\
a_{n+1} & =\sqrt{4 a_{n}+\mathrm{I}}-\left(a_{n}+\mathrm{I}\right) .
\end{aligned}
$$

Questa successione è decrescente e tendente a zero. Sopra ogni segmento $\left(a_{n+1}, a_{n}\right)$, dell'asse $x$, preso come ipotenusa, costruiamo il triangolo rettangolo isoscele, con il vertice dell'angolo retto dalla parte degli $y$ positivi. L'insieme di tutti i cateti di questi triangoli costituirà la nostra curva $C_{0}$, la quale unirà cosi il punto $(0,0)$ al punto $(\mathrm{I}, \mathrm{O})$; avrà lunghezza uguale a $\sqrt{2}$ e risulterà composta di un'infinità numerabile di segmenti rettilinei paralleli alle bisettrici degli angoli degli assi $x$ e $y$. Nel punto $(0,0)$ la $C_{o}$ ammette tangente e questa tangente è data dall'asse delle $x$. Infatti, nel vertice di $C_{0}$ di ascissa $\frac{a_{n}+a_{n+1}}{2}$, l'ordinata è data da $\frac{a_{n}-a_{n+1}}{2}$ ed è, per la definizione delle $a_{n}$,

$$
\frac{a_{n}-a_{n+\mathrm{I}}}{2}=\left(\frac{a_{n}+a_{n+\mathrm{I}}}{2}\right)^{2}
$$

tale vertice è perció sulla parabola $y=x^{2}$. 
Nel punto $(0,0)$ è $\theta_{0}=0$, onde

$$
F_{1}(0,0, \cos 0, \operatorname{sen} 0)=\frac{\sqrt{2}(2 \sqrt{2}-3)}{(\sqrt{2}-I)^{3}}<0 \text {. }
$$

Per altro, essendo in ogni punto di $C_{o}$ dove esiste la tangente [escluso il punto $(0,0)$, $\cos \theta_{0}=\frac{I}{\sqrt{2}}, \operatorname{sen} \theta_{0}= \pm \frac{I}{\sqrt{2}}$, si ha, in questi punti,

$$
E\left(x_{0}, y_{0} ; \cos \theta_{o}, \operatorname{sen} \theta_{0} ; \cos \bar{\theta}, \operatorname{sen} \bar{\theta}\right)=\frac{\mathrm{I}}{\sqrt{2}-\cos \bar{\theta}}-2 \cos \bar{\theta}=\frac{(\sqrt{2} \cos \bar{\theta}-\mathrm{I})^{2}}{\sqrt{2}-\cos \bar{\theta}} \geq 0
$$

ed essendo sempre, per tutte le coppie $\left(x^{\prime}, y^{\prime}\right)$ numeri non ambedue nulli, $F>0$, l'Osservazione $\mathrm{II}^{\mathrm{a}}$ mostra che sulla $C_{\mathrm{o}}$ l'integrale della $F$ è semicontinuo inferiormente.

\section{$\int 6$.}

\section{Condizioni necessarie per la semicontinuità.}

a) La semicontinuità in tutto il campo.

29. Vogliamo dimostrare la seguente proposizione:

Condizione necessaria affinche su ogni curva $\left(\mathrm{n}^{\circ} 3\right)$ di $A$ l'integrale $I_{C}$ sia funzione semicontinua inferiormente, è che si abbia

$$
F_{\mathrm{I}}\left(x ; y, x^{\prime}, y^{\prime}\right) \gg 0
$$

per ogni coppia di numeri $x^{\prime}, y^{\prime}$ non ambedue nulli $e$ in tutti $i$ punti $(x, y)$ interni al campo $A$ o limiti di punti interni.

Supponiamo che esistano un punto $\left(x_{0}, y_{0}\right)$, interno al campo $A$, ed un angolo $\theta_{0}$ tali da rendere soddisfatta la disuguaglianza

$$
F_{\mathrm{r}}\left(x_{0}, y_{0}, \cos \theta_{0}, \operatorname{sen} \theta_{0}\right)<0 .
$$

Per la continuità della funzione $F_{1}$ rispetto all'insieme delle quattro variabili $x, y, x^{\prime}, y^{\prime}$, considerato un numero positivo $n$, comunque scelto, purchè soddisfacente alla disuguaglianza

$$
F_{s}\left(x_{0}, y_{0}, \cos \theta_{0}, \operatorname{sen} \theta_{0}\right)<-n,
$$

è possibile determinare un'altro numero positivo $\delta$ tale che sia

$$
F_{1}(x, y, \cos \theta, \operatorname{sen} \theta)<-n
$$

ogni qualvolta risultino soddisfatte le disuguaglianze

$$
\left|x-x_{0}\right| \leq \delta, \quad\left|y-y_{0}\right| \leq \delta,
$$

Si intende che $\delta$ deve esser anche tale che $\mathrm{i}$ punti $(x y)$ soddisfacenti alle due prime disuguaglianze risultino tutti interni al campo $A$. 
Per il punto $\left(x_{0}, y_{0}\right)$ si conduca un segmento rettilineo $P_{\mathrm{o}} P_{\mathrm{r}}$, di lunghezza $l \leqslant \delta$, che faccia con la direzione positiva dell'asse delle $x$ l'angolo $\theta_{\circ}$; si scelga poi un angolo positivo $\alpha<\frac{\pi}{4}$, soddisfacente alla disuguaglianza $\alpha<\delta$. Dopo ciò si divida il segmento $P_{\mathrm{o}} P_{\mathrm{I}}$ in $n$ parti uguali e su ciascuna di esse, presa come base e sempre dalla stessa parte di $P_{\mathrm{o}} P_{\text {}}$, si costruisca il triangolo isoscele avente gli angoli alla base uguali ad $x$. L'insieme di tutte le basi di questi triangoli darà allora il segmento $P_{0} P_{1}$; l'insieme invece di tutti gli altri lati (fra loro uguali) darà una curva, che indicheremo con $C_{n}$, formata di $2 n$ segmenti rettilinei e con gli estremi in $P_{\mathrm{o}}$ e $P_{\mathrm{r}}$.

Preso un punto qualunque $M$ di $C_{n}$, indichiamo con $P$ la sua proiezione ortogonale su $P_{0} P_{1}$ e con $s$ la lunghezza del segmento $P_{0} P$. Scegliendo $s$ come parametro di una rappresentazione analitica simultanea delle curve $P_{0} P_{i}$ e $C_{n}$, la quale faccia corrispondere $P$ ad $M$, avremo, per $P_{\mathrm{o}} P_{\mathrm{r}}$, le equazioni

e per $C_{n}$

$$
\begin{array}{lll}
x=x(s), & y=y(s), & (0 \leq s \leq l), \\
x=x_{n}(s), & y=y_{n}(s) & (0 \leq s \leq l) ;
\end{array}
$$

e sard, indicando con $s_{n}$ la lunghezza dell'arco di $C_{n}$ corrispondente all'intervallo (o, $s$ ),

$$
\left\{\begin{aligned}
x^{\prime}(s) & =\cos \theta_{0}, \quad y^{\prime}(s)=\operatorname{sen} \theta_{0}, \\
x_{n}^{\prime}(s)=\frac{d x_{n}}{d s_{n}} \cdot \frac{d s_{n}}{d s} & =\frac{\cos \left(\theta_{0} \pm \alpha\right)}{\cos \alpha}, \quad y_{n}^{\prime}(s)=\frac{\operatorname{sen}\left(\theta_{0} \pm \alpha\right)}{\cos \alpha} .
\end{aligned}\right.
$$

Consideriamo allora la differenza

$$
\Delta I=\int_{C_{n}} F d s_{n}-\int_{P_{0} P_{1}} F d s=\int_{0}^{l}\left\{F\left(x_{n}, y_{n}, x_{n}^{\prime}, y_{n}^{\prime}\right)-F\left(x, y, x^{\prime}, y^{\prime}\right)\right\} d s \text {. }
$$

Essa puó scriversi

$$
\begin{aligned}
\Delta I & =\int_{0}^{l}\left\{F\left(x_{n}, y_{n}, x_{n}^{\prime}, y_{n}^{\prime}\right)-F\left(x, y, x_{n}^{\prime}, y_{n}^{\prime}\right)\right\} d s \\
& +\int_{0}^{l}\left\{F\left(x, y, x_{n}^{\prime}, y_{n}^{\prime}\right)-F\left(x, y, x^{\prime}, y^{\prime}\right) d s .\right.
\end{aligned}
$$

Ma per la formula stabilita al $\mathrm{n}^{\mathrm{o}} 9$, è, tenendo conto delle (24);

$$
\begin{gathered}
F\left(x, y, x_{n}^{\prime}, y_{n}^{\prime}\right)=F\left[x, y, \frac{\cos \left(\theta_{0} \pm \alpha\right)}{\cos \alpha}, \frac{\operatorname{sen}\left(\theta_{0} \pm \alpha\right)}{\cos \alpha}\right] \\
=\frac{\mathrm{I}}{\cos \alpha} F\left[x, y, \cos \left(\theta_{\mathrm{o}} \pm \alpha\right), \operatorname{sen}\left(\theta_{0} \pm x\right)\right]
\end{gathered}
$$

$=\frac{I}{\cos \alpha}\left\{\cos \left(\theta_{0} \pm \alpha\right) F_{x^{\prime}}\left(x, y, \cos \theta_{0}, \operatorname{sen} \theta_{0}\right)+\operatorname{sen}\left(\theta_{0} \pm \alpha\right) F_{y^{\prime}}\left(x, y, \cos \theta_{0} \operatorname{sen} \theta_{0}\right)\right.$

$$
\begin{gathered}
\left.+\int_{\theta_{0}}^{\theta_{0} \pm \alpha} F_{\mathrm{x}}(x, y, \cos \tau, \operatorname{sen} \tau) \operatorname{sen}\left(\theta_{0} \pm \alpha-\tau\right) d \tau\right\} \\
\quad=x_{n}^{\prime} F_{x^{\prime}}\left(x, y, x^{\prime}, y^{\prime}\right)+y_{n}^{\prime} F_{y^{\prime}}\left(x, y, x^{\prime}, y^{\prime}\right) \\
+\frac{\mathrm{I}}{\cos \alpha} \int_{\theta_{0}}^{\theta_{0} \pm \alpha} F_{\mathrm{I}}(x, y, \cos \tau, \operatorname{sen} \tau) \operatorname{sen}\left(\theta_{0} \pm \alpha-\tau\right) d \tau .
\end{gathered}
$$


Sostituendo nell'espressione di $\Delta I$, si ha, per essere

$$
\begin{gathered}
F\left(x, y, x^{\prime}, y^{\prime}\right)=x^{\prime} F_{x^{\prime}}\left(x, y, x^{\prime}, y^{\prime}\right)+y^{\prime} F_{y^{\prime}}\left(x, y, x^{\prime}, y^{\prime}\right), \\
\Delta I=\int_{0}^{l}\left\{F\left(x_{n}, y_{n}, x_{n}^{\prime}, y_{n}^{\prime}\right)-F\left(x, y, x_{n}^{\prime}, y_{n}^{\prime}\right)\right\} d s \\
+\int_{0}^{l}\left\{\left(x_{n}^{\prime}-x^{\prime}\right) F_{x^{\prime}}\left(x, y, x^{\prime}, y^{\prime}\right)+\left(y_{n}^{\prime}-y^{\prime}\right) F_{y^{\prime}}\left(x, y, x^{\prime}, y^{\prime}\right)\right\} d s \\
+\frac{\mathrm{I}}{\cos \alpha} \int_{0}^{l} d s \int_{\theta_{0}}^{\theta_{0}+x} F_{1}(x, y, \cos \tau, \operatorname{sen} \tau) \operatorname{sen}\left(\theta_{0} \pm \alpha-\tau\right) d \tau .
\end{gathered}
$$

Osservando ora che, per la (23), è ${ }^{29}$ )

$$
\begin{gathered}
\int_{\theta_{0}}^{\theta_{0} \pm \alpha} F_{\mathrm{I}}(x, y, \cos \tau, \operatorname{sen} \tau) \operatorname{sen}\left(\theta_{0} \pm \alpha-\tau\right) d \tau \\
<-\eta \int_{\theta_{0}}^{\theta_{0} \pm \alpha} \operatorname{sen}\left(\theta_{0} \pm \alpha-\tau\right) d \tau=-n(\mathrm{I}-\cos \alpha),
\end{gathered}
$$

si ottiene

$$
\begin{gathered}
\Delta I<\int_{0}^{l}\left\{F\left(x_{n}, y_{n}, x_{n}^{\prime}, y_{n}^{\prime}\right)-F\left(x, y, x_{n}^{\prime}, y_{n}^{\prime}\right)\right\} d s \\
+\int_{0}^{l}\left\{\left(x_{n}^{\prime}-x^{\prime}\right) F_{x^{\prime}}\left(x, y, x^{\prime}, y^{\prime}\right)+\left(y_{n}^{\prime}-y^{\prime}\right) F_{y^{\prime}}\left(x, y, x^{\prime}, y^{\prime}\right)\right\} d s \\
-n l(\mathrm{I}-\cos \alpha) .
\end{gathered}
$$

Ora, siccome, per $n \rightarrow \infty, x_{n}(s)$ e $y_{n}(s)$ tendono uniformemente, in tutto l'intervallo $(o, l)$, a $x(s), y(s)$, rispettivamente, ed è sempre $x_{n}^{\prime 2}+y_{n}^{\prime 2}=\frac{\mathrm{I}}{\cos ^{2} \alpha}$; dalla continuità della funzione $F$ scende che, preso ad arbitrio un $\sigma>0$, per $n$ sufficientemente grande, il primo integräle della precedente disuguaglianza risulta minore di $\sigma$.

Ugualmente minore di $\sigma$ risulta, per $n$ sufficientemente grande, il secondo integrale, in forza del lemma del $\mathrm{n}^{\circ}$ 8. Possiamo quindi concludere che, per ogni $n$ maggiore di un certo $\bar{n}$, è

$$
\Delta I=\int_{C_{n}} F d s_{n}-\int_{P_{0} P_{1}} F d s<-\frac{n l(1-\cos \alpha)}{2} .
$$

E siccome, per $n \rightarrow \infty$, la curva $C_{n}$ tende uniformemente al segmento $P_{0} P_{1}$, resta dimostrato che l'integrale della $F$ sul segmento $P_{0} P_{\mathrm{I}}$ non è funzione semicontinua inferiormente. Ciò prova che, in ogni punto interno al campo $A$, deve esser verificata la (22) se si vuole che si abbia sempre la semicontinuita inferiore di $I_{C}$.

La (22), essendo verificata in ogni punto interno, deve poi necessariamente essere

29) Aumentando, se occorre, $\theta_{0} \pm \alpha \mathrm{di} \pm 2 \pi$, si potrà sempre far in modo che la quantità sotto l'integrale che stiamo per scrivere risulti sempre di un segno nell'intervallo d'integrazione. 
soddisfatta anche in ogni punto della frontiera di $A$ che sia limite di punti interni: ciò che è immediata conseguenza della continuità della $F_{\mathrm{r}}$.

3o. Un ulteriore condizione necessaria, per la semicontinuità inferiore di $I_{C}$ su ogni curva del campo $A$, è data dall'uguaglianza

$$
F_{x^{\prime} y}\left(x_{0}, y_{0}, \cos \theta \operatorname{sen} \theta\right)=F_{y^{\prime} x}\left(x_{0}, y_{0}, \cos \theta, \operatorname{sen} \theta\right) \text {, }
$$

la quale deve essere soddisfatta, per tutti $i$ valori di $\theta$, in ogni punto $\left(x_{0}, y_{0}\right)$, interno ad $A$, in cui sia

$$
F_{\mathrm{s}}\left(x_{0}, y_{0}, x^{\prime}, y^{\prime}\right) \equiv 0
$$

identicamente rispetto a tutte le possibili coppie $\left(x^{\prime}, y^{\prime}\right)$.

Consideriamo uno dei punti $\left(x_{0}, y_{0}\right)$ ora indicati, e ammettiamo che esista un valore di $\theta, \theta_{o}$, tale che sia

$$
F_{x^{\prime} y}\left(x_{0}, y_{0}, \cos \theta_{0}, \operatorname{sen} \theta_{0}\right) \neq F_{y^{\prime} x}\left(x_{0}, y_{0}, \cos \theta_{0}, \operatorname{sen} \theta_{0}\right) .
$$

Indichiamo con $\gamma$ un cerchio del piano $(x, y)$ avente $\left(x_{0}, y_{0}\right)$ sulla circonferenza e tale che tutti i suoi punti siano punti interni ad $A$. La sua circonferenza la indicheremo con $\gamma^{\prime}$, il suo raggio, con $r$.

Detto $M$ il modulo della differenza

$$
F_{x^{\prime} y}\left(x_{0}, y_{0}, \cos \theta_{0}, \operatorname{sen} \theta_{0}\right)-F_{y^{\prime} x}\left(x_{0}, y_{0}, \cos \theta_{0}, \operatorname{sen} \theta_{0}\right),
$$

indichiamo con $\delta$ un numero positivo tale che in tutto il cerchio del piano $(x, y)$ di centro $\left(x_{0}, y_{0}\right)$ e raggio $\delta$ sia

$$
\left|F_{x^{\prime} y}\left(x, y, \cos \theta_{0}, \operatorname{sen} \theta_{0}\right)-F_{y^{\prime} x}\left(x, y, \cos \theta_{0}, \operatorname{sen} \theta_{0}\right)\right| \geqslant \frac{M}{2} .
$$

Intenderemo che sia $r \leq \frac{\delta}{2}$. Sceglieremo anche il senso positivo sulla circonferenza $\gamma^{\prime}$ in modo che l'integrale

$$
\int_{\gamma^{\prime}}\left\{x^{\prime} F_{x^{\prime}}\left(x, y, \cos \theta_{0}, \operatorname{sen} \theta_{0}\right)+y^{\prime} F_{y^{\prime}}\left(x, y, \cos \theta_{0}, \operatorname{sen} \theta_{0}\right)\right\} d s,
$$

il cui valore, per il teorema di Green, è dato da

$$
\pm \iint_{Y}\left\{F_{x^{\prime} y}\left(x, y, \cos \theta_{0}, \operatorname{sen} \theta_{0}\right)-F_{y^{\prime} x}\left(x, y, \cos \theta_{0}, \operatorname{sen} \theta_{0}\right)\right\} d x d y
$$

risulti negativo. Sarà allora, per la precedente disuguaglianza,

$$
\int_{r^{\prime}}\left\{x^{\prime} F_{x^{\prime}}\left(x, y, \cos \theta_{0}, \operatorname{sen} \theta_{0}\right)+y^{\prime} F_{y^{\prime}}\left(x, y, \cos \theta_{0}, \operatorname{sen} \theta_{0}\right\} d s \leq-\frac{M}{2} \pi r^{2} .\right.
$$

Ciò premesso, consideriamo la curva $\gamma_{n}^{\prime}$ che si ottiene percorrendo $n$ volte la cir- 
conferenza $\gamma^{+}$, nel senso positivo gii stabilito, e l'integrale

$$
\int_{Y_{n}^{\prime}} F\left(x, y, x^{\prime}, y^{\prime}\right) d s \text {. }
$$

Applicando la formula del $\mathrm{n}^{\circ} 9$, abbiamo

$$
\begin{aligned}
\int_{Y_{n}^{\prime}} F d s= & \int_{Y_{n}^{\prime}}\left\{x^{\prime} F_{x^{\prime}}\left(x, y, \cos \theta_{0}, \operatorname{sen} \theta_{0}\right)+y^{\prime} F_{y^{\prime}}\left(x, y, \cos \theta_{0}, \operatorname{sen} \theta_{0}\right)\right\} d s \\
& +\int_{r_{n}^{\prime}} d s \int_{\theta_{0}}^{\theta} F_{\mathrm{r}}(x, y, \cos \alpha, \operatorname{sen} \alpha) \operatorname{sen}(\theta-\alpha) d \alpha
\end{aligned}
$$

dove $\theta$ risulta definito dalle $x^{\prime}(s)=\cos \theta, y^{\prime}(s)=\operatorname{sen} \theta$, ed è preso in modo che $\operatorname{sen}(\theta-\alpha)$ abbia segno costante in tutto Pintervallo $\left(\theta_{0}, \theta\right)$. Per l'ultima disuguaglianza scritta abbiamo

$$
\int_{Y_{n}^{\prime}} F d s \leqslant-\frac{M}{2} n \pi r^{2}+\int_{\substack{Y_{n}^{\prime} \\ r_{1}}} d s \int_{\theta_{0}}^{\theta} F_{\mathrm{I}}(x, y, \cos \alpha, \operatorname{sen} \alpha) \operatorname{sen}(\theta-\alpha) d \alpha,
$$

ed anche, indicando con $F_{1}(r)$ il massimo valore di $F_{1}(x, y, \cos \alpha, \operatorname{sen} \alpha)$ nel cerchio $\gamma$ e'per ogni $\alpha$ (massimo che $\dot{\mathrm{e}} \gg 0$, per il $\mathrm{n}^{\circ} 29$ ),

$$
\int_{\gamma_{n}^{\prime}} F d s \leq-\frac{M}{2} n \pi r^{2}+4 \pi r n F_{\mathrm{I}}(r)=\frac{\pi}{2} r^{2} n\left(-M+16 \frac{F_{1}(r)}{2 r}\right) .
$$

Se indichiamo con $\lambda$ la distanza dal punto $\left(x_{0}, y_{0}\right)$ del punto del cerchio $\gamma$ in cui $F_{\mathrm{r}}$ assume il valore $F_{1}(r)$, abbiamo

$$
\frac{F_{\mathrm{I}}(r)}{2 r} \leqslant \frac{F_{\mathrm{I}}(r)}{\lambda}
$$

e siccome in $\left(x_{0}, y_{0}\right)$ la funzione $F_{\mathrm{t}}$ è identicamente nulla, mentre negli altri punti è sempre $F_{\mathrm{I}} \gg \mathrm{o}\left(\mathrm{n}^{\circ}\right.$ precedente), in $\left(x_{0}, y_{0}\right)$ tale funzione ha un minimo ed è, per $\lambda \rightarrow 0$,

$$
\frac{F_{1}(r)}{\lambda} \rightarrow 0
$$

Dunque, per ogni $r$ minore di un certo $r_{\mathrm{r}}$, è

$$
\int_{r_{n}^{\prime}} F d s<-\frac{\pi}{4} M r^{2} n
$$

Questa disuguaglianza prova che, comunque si prenda una curva $C_{\mathrm{o}}$ passante per $\left(x_{0}, y_{o}\right)$, su tale curva $I_{C}$ non puó essere semicontinuo inferiormente. Ed infatti, scelto ad arbitrio un numero positivo $N$ e preso un $\rho>0$ e piccolo quanto vuolsi, si con- 
sideri la curva $C$ composta: $I^{\circ}$ ) dell'arco di $C_{0}$ che dal primo estremo di questa curva va al punto $\left.\left(x_{0}, y_{0}\right) ; 2^{\circ}\right)$ della curva $\gamma_{n}^{\prime}$, dove si prenderà $r<\frac{p}{4}$, oltrechè soddisfacente alle condizioni dianzi indicate, e $n>\frac{4 N}{\pi M r^{2}} ; 3^{\circ}$ ) dell'arco della $C_{0}$ che dal punto $\left(x_{0}, y_{0}\right)$ va al secondo estremo della curva stessa. La curva cosi definita appartiene ordinatamente all'intorno $(\rho)$ della $C_{0}$ e soddisfa alla disuguaglianza

$$
\int_{C} F d \sigma<\int_{C_{\mathrm{o}}} F d s-N,
$$

dove con $d \sigma$ abbiamo indicato l'elemento d'arco di $C$ e con $d s$ quello della $C_{0}$. Questa disuguaglianza, per essere $N>0$ e $\rho$ comunque piccolo, contraddice alla semicontinuità inferiore dell'integrale della $F$ sulla curva $C_{0}$.

31. Da quanto si è stabilito ai $\mathrm{n}^{\mathrm{i}} 29$ e 30 risulta la proposizione:

Condizione necessaria affinche l'integrale $I_{C}$ sia funzione semicontinua inferiormente, su ogni curva completamente interna al campo $A$, è che, in ogni punto iuterno a questo campo, sia sempre $F_{1} \supseteq 0$, per tutie le coppie $\left(x^{\prime}, y^{\prime}\right)$ di numeri non ambedue nulli, ed inoltre che, in ogni punto interno ad $A$ in cui si abbia $F_{\mathrm{I}} \equiv 0$, per tutte le coppie $\left(x^{\prime}, y^{\prime}\right)$ dette, sia $F_{x^{\prime} y} \equiv F_{y^{\prime} x}$ pure per tutte le stesse coppie $\left(x^{\prime}, y^{\prime}\right)$.

In particolare possiamo dire: $\operatorname{Se} P(x, y) Q(x, y)$ sono due funzioni continue nel campo $A$ insieme con le due derivate $\frac{\partial P}{\partial y}, \frac{\partial Q}{\partial x}$, condizione necessaria affinchè, su ogni curva completamente interna ad $A$, lintegrale

$$
\int_{C}\left\{x^{\prime} P(x, y)+y^{\prime} Q(x, y)\right\} d s
$$

sia funzione semicontinua inferiormente o superiormente (oppure funzione continua) è che, in ogni punto interno o limite di punti interni al campo, sia $\frac{\partial P}{\partial y}=\frac{\partial Q}{\partial x}$.

\section{$\$ 7$.}

Condizioni necessarie per la semicontinuità.

b) La semicontinuità su una data curva.

32. Occupiamoci ora di determinare le condizioni necessarie affinchè si abbia la semicontinuita di $I_{C}$ su una determinata curva.

Cominciamo col dimostrare la seguente proposizione:

Condizione necessaria affinchè su una data curva $C_{0}$, completamente interna al campo $A$ e dotata ovunque di tangente variabile in modo continuo, lintegrale $I_{C}$ sia funzione 
semicontinua inferiormente, $\dot{e}$ che in ogni punto $\left(x_{0}, y_{0}\right)$ della curva si abbia

$$
F_{\mathrm{I}}\left(x_{0}, y_{0}, \cos \theta_{0}, \operatorname{sen} \theta_{0}\right) \supseteq 0,
$$

dove $\theta_{0}$ indica l'angolo che la direzione positiva della tangente alla curva stessa forma con la direzione positiva dell'asse delle $x$.

Supponiamo che in un punto $\left(x_{0}, y_{0}\right)$ della $C_{0}$ sia

$$
F_{\mathrm{r}}\left(x_{0}, y_{0}, \cos \theta_{0}, \operatorname{sen} \theta_{0}\right)<0 .
$$

Indicato, come al $n^{\circ} 29$, con $n$ un numero positivo soddisfacente alla disuguaglianza

$$
F\left(x_{0}, y_{0}, \cos \theta_{0}, \operatorname{sen} \theta_{0}\right)<-n,
$$

sia $\delta$ un altro numero positivo tale che risulti

ogni qualvolta si abbia

$$
F_{\mathrm{I}}(x, y, \cos \omega, \operatorname{sen} \omega)<-\eta
$$

$$
\begin{gathered}
\left|x-x_{0}\right| \leq 2 \delta, \quad\left|y-y_{0}\right| \leq 2 \delta, \\
\left|\omega-\theta_{0}\right| \leq 2 \delta .
\end{gathered}
$$

Si indichi ancora con $\alpha$ un angolo positivo $<\delta$ e $<\frac{\pi}{4}$.

Ciò premesso, si consideri un arco $\gamma$ della curva $C_{0}$ che abbia come primo estremo il punto $\left(x_{0}^{\prime}, y_{0}\right)$, lunghezza uguale o minore di $\delta$ e sia tale che l'angolo $\theta$ relativo alla sua tangente in un punto qualunque soddisfi sempre alla

$$
\left|\theta-\theta_{0}\right|<\delta \text {. }
$$

Indicheremo con $P_{\mathrm{o}}$ e $P_{\mathrm{I}}$ gli estremi di quest'arco e con la stessa lettera $\gamma$ la sua lunghezza.

Inscriviamo in $\gamma$ una poligonale $\mathrm{II}$, completamente interna al campo $A$, avente gli estremi in $P_{\mathrm{o}}$ e $P_{\mathrm{r}}$ ed $\mathrm{i}$ vertici susseguentisi su $\gamma$ nello stesso ordine fissato dal verso dell'arco.

Siano $l_{0}, l_{1}, l_{2}, \ldots, l_{r}$ i lati successivi di questa poligonale e consideriamone uno qualunque $l_{p}$. Se indichiamo con $x_{p}, y_{p}$ le coordinate del primo estremo di $l_{p}$ e con $\omega_{p}$ l'angolo che questo lato forma con la direzione positiva dell'asse delle $x$, la disuguaglianza

$$
F_{x}(x, y, \cos \omega, \operatorname{sen} \omega)<-n
$$

risulta verificata tosto che sia

$$
\left|x-x_{p}\right| \leq \delta, \quad\left|y-y_{p}\right| \leq \delta,
$$

E poichè la lunghezza $l_{p}$ è $\leqslant \gamma \leqslant \delta$, per quanto si è dimostrato al $\mathrm{n}^{0} 29$ possiamo 
asserire che, comunque piccolo si fissi un intorno di $l_{p}$, è sempre possibile di costruire una poligonale $\Pi_{p}$, completamente interna al campo $A$, avente gli estremi comuni con $l_{p}$, appartenente ordinatamente a tale intorno e soddisfacente alla disuguaglianza

$$
\int_{\Pi_{p}} F d s_{\Pi}-\int_{l_{p}} F d s_{l}<-\frac{n l_{p}(\mathrm{I}-\cos \alpha)}{2} .
$$

Siccome $l_{p}$ è uno qualunque dei lati della poligonale II, abbiamo che, fissato ad arbitrio un intorno di li, è sempre possibile costruire un'altra poligonale $\mathrm{r}$, appartenente ordinatamente a tale intorno, completamente interna ad $A$ e soddisfacente alla disuguaglianza

$$
\int_{\Gamma} F d s_{\Gamma}-\int_{\Pi} F d s_{\Pi}<-\frac{\eta \Pi(I-\cos \alpha)}{2},
$$

dove abbiamo indicato con II anche la lunghezza della poligonale II.

Ora, potendosi determinare la poligonale II in modo che essa appartenga ordinatamente ad un intorno comunque piccolo di $\gamma$, e poichè al tendere a zero di questo intorno la lunghezza II tende a quella $\gamma$, l'integrale della $F$ esteso a II tende a quello della stessa funzione esteso a $\gamma^{30}$ ) e possiamo concludere che, fissato ad arbitrio un intorno di $\gamma$, è sempre possibile determinare una poligonale $\Gamma$ appartenente ordinatamente a tale intorno, completamente interna ad $A$ e tale da rendere soddisfatta la disuguaglianza

$$
\int_{\Gamma} F d s_{\Gamma}-\int_{\gamma} F d s<-\frac{n \gamma(\mathrm{I}-\cos \alpha)}{4} .
$$

Ció prova che sulla curva $C_{0}$, ammessa l'ipotesi dell'esistenza del punto $\left(x_{0} y_{0}\right)$, indicato in principio, non puó sussistere la semicontinuita inferiore dell'integrale $I_{C}$.

Osservazione. - La condizione ora dimostrata necessaria per la semicontinuità inferiore dell'integrale $I_{C}$ sulla curva $C_{0}$, è necessaria anche se questa curva, pur sempre appartenendo al campo $A$, non è completamente interna ad esso, purchè nessun arco della $C_{\mathrm{o}}$ sia tuțto costituito di punti della frontiera di $A$, od anche purchè : $\mathbf{I}^{\circ}$ ), fissato ad arbitrio un intorno $(p)$ della $C_{o}$, si possa sempre trovare una poligonale $\Pi$, ordinatamente appartenente a questo intorno, completamente interna ad $A$ e tale che la sua lunghezza differisca da quella della curva $C_{o}$ per meno di $p ; 2^{\circ}$ ) fissato un $\delta>0$ qualsiasi ed un punto $M$ pure qualsiasi di $A$, si possa sempre determinare un cerchio di centro $M$ in modo che ogni suo punto appartenente ad $A$ possa congiungersi con $M$ mediante una curva continua, pure appartenente ad $A$, di lunghezza $<\delta$.

$\left.3^{\circ}\right)$ V. L. Tonell, Successioni di curve e derivazione per serie (Rend. della R. Accademia dei Lincei, vol. XXV, $1^{0}$ semestre 1916, pp. 22-30), $\mathrm{n}^{0} 6$. 
Per i campi che comunemente si considerano nel Calcolo delle Variazioni, le condizioni $\left.\mathrm{I}^{\circ}\right)$ e $2^{\circ}$ ) risultano soddisfatte per tutte le curve.

33. Se la curva $C_{0}$ non soddisfa alla condizione ammessa al $\mathbf{n}^{\circ}$ precedente, vale a dire, non ha in ogni punto tangente variabile in modo continuo, la condizione che sia sempre verificata la

$$
F_{\mathrm{I}}\left(x_{0}, y_{0}, \cos \theta_{0}, \operatorname{sen} \theta_{0}\right) \supseteq 0
$$

là dove la tangente esiste, non è più necessaria. Ciò risulta senz'altro da quanto si è detto al $\mathrm{n}^{\circ} 28$ (Osservazione III). Per il caso qui contemplato possiamo peró dimostrare la seguente proposizione:

Condizione necessaria affinchè, su una data curva $C_{0}$, completamente interna al. campo $A$, l'integrale $I_{C}$ sia funzione semicontinua inferiormente, è che abbia misura nulla l'insieme dei punti $\left(x_{0}, y_{\mathrm{o}}\right)$ di $C_{\mathrm{o}}$ nei quali non è verificata la disuguaglianza

$$
F_{1}\left(x_{0}, y_{0}, \cos \theta_{0}, \operatorname{sen} \theta_{0}\right) \gg 0,
$$

dove $\theta_{0}$ indica l'angolo che la direzione positiva della tangente alla curva stessa, là dove questa tangente esiste, forma con la direzione positiva dell' asse $x$.

Supponiamo che la misura (contata sulla curva) dei punti di $C_{0}$ nei quali la tangente alla curva esiste ed è $F_{1}\left(x_{0}, y_{0}, \cos \theta_{0}, \operatorname{sen} \theta_{0}\right)<0$ non sia nulla. Indicatala con $2 \mu$, possiamo trovare un $n>0$ e un gruppo chiuso $E$ di questi punti, che abbia misura maggiore di $\mu \mathrm{e}$ in ogni punto del quale sia $F_{1}\left(x_{0}, y_{0}, \cos \theta_{0}, \sin \theta_{0}\right)<-\frac{3}{2} n$.

Per la continuita della funzione $F_{1}$, possiamo determinare un numero positivo, $\delta$ in modo che, se $(x y)$ è un punto di $A$, risulti sempre soddisfatta la disuguaglianza

ogni qualvolta sia

$$
F_{1}(x, y, \cos \omega, \operatorname{sen} \omega)<-n
$$

$$
\begin{gathered}
\left|x-\bar{x}_{\mathrm{o}}\right| \leq 3 \delta, \quad\left|y-\bar{y}_{\mathrm{o}}\right| \leq 3 \delta \\
\left|\omega-\bar{\theta}_{\mathrm{o}}\right| \leq 2 \delta,
\end{gathered}
$$

e ció per tutti i punti $\left(\bar{x}_{0}, \overline{y_{0}}\right)$ di $E$. Si indichi poi anche qui con $\alpha$ un angolo positivo minore di $\delta$ e di $\frac{\pi}{4}$.

Dividiamo la curva $C_{o}$ in $n$ archi tutti della stessa lunghezza $\frac{L_{0}}{n}$, dove $L_{0}$ indica la lunghezza della $C_{o}$, e congiungiamo gli estremi di ogni arco con un segmento rettilineo. Avremo cosi una poligonale $\boldsymbol{\Pi}_{n}$ inscritta in $C_{o}$; e intenderemo che $n$ sia sufficientemente grande perchè questa poligonale risulti completamente interna al campo $A$. Detta $L_{n}$ la lunghezza della $\mathbf{I I}_{n}$; risulta che, per $n \rightarrow \infty$, la $\boldsymbol{\Pi}_{n}$ tende uniformemente alla $C_{\mathrm{o}}$ e $L_{n}$ tende a $L_{\mathrm{o}}$. Indicata poi con $s$ la lunghezza dell'arco della $C_{\mathrm{o}}$, a partire dal suo primo estremo $P_{\mathrm{o}}$, e con $s_{n}$ quella dell'arco della $\Pi_{n}$, pure a partire da $P_{0}$, e 
fatto corrispondere ad ogni punto $P$ di $C_{0}$, individuato dal valore $s$ del parametro, il punto $P_{n}$ di $I_{n}$ individuato dal valore del parametro $s_{n}=\frac{L_{n}}{L_{0}} s$, abbiamo che, preso ad arbitrio un $\varepsilon>0$, possiamo determinare ${ }^{\mathbf{3}}$ ) un indice $\bar{n}$ tale che, per ogni $n>\bar{n}$ : di $\delta$;

$\left.I^{\circ}\right)$ la distanza fra due punti corrispondenti qualsiasi $P$ e $P_{n}$ sia sempre minore

$\left.2^{\circ}\right)$ si abbia

$$
\left|\int_{\Pi_{n}} F d s_{n}-\int_{C_{0}} F d s\right|<\varepsilon
$$

$\left.3^{\circ}\right)$ l'insieme $E_{n}$ dei punti $\widetilde{P}_{n}$ di $\Pi_{n}$ corrispondenti ai punti $\bar{P}$ di $E$ ed in cui è $\left|\bar{\theta}_{n}-\bar{\theta}_{0}\right|<\delta-$ dove $\bar{\theta}_{n}$ è l'angolo che da la direzione del lato di $\mathrm{II}_{n}$ a cui $\bar{P}_{n}$ appartiene - risulti di misura (contata sulla $\Pi_{n}$ ) maggiore di $\mu$.

Sia $n>\bar{n}$ e $E_{n}^{\prime}$ un insieme chiuso qualsiasi contenuto in $E_{n}$ e di misura maggiore di $\mu$. Se $\bar{P}_{n}^{\prime}$ è un punto qualunque di $E_{n}^{\prime}$, possiamo determinare il massimo intervallo che lo contiene, che ha gli estremi distanti da questo punto non più di $\frac{\delta}{2}$ e che appartiene completamente al lato di $\Pi_{n}$ di cui fa parte il punto $\bar{P}_{n}^{\prime}$ stesso. Fra gli intervalli cosi determinati, possiamo sceglierne un numero finito che ricoprano tutto l'insieme $E_{n}^{\prime}$; e ordinatili a piacere possiamo sopprimere in ciascuno di essi quelle parti che eventualmente appartenessero agli intervalli del gruppo stesso di indice minore. Otteniamo cosi dei segmenti non sovrapponentesi $l_{n}^{(1)}, l_{n}^{(2)}, \ldots, l_{n}^{\left(m_{n}\right)}$, appartenenti a $\Pi_{n}$, ognuno di ampiezza $\leqslant \delta$ e tali che, se $P_{n}^{(r)}\left(x_{n}^{(r)}, y_{n}^{(r)}\right)$ indica il primo estremo di $l_{n}^{(r)}$, risulta

tutte le volte che è

$$
F_{\mathrm{I}}(x, y, \cos \omega, \operatorname{sen} \omega)<-n
$$

$$
\begin{gathered}
\left|x-x_{n}^{(r)}\right| \leq \delta, \quad\left|y-y_{n}^{(r)}\right| \leq \delta, \\
\left|\omega-\theta_{n}^{(r)}\right| \leq \delta,
\end{gathered}
$$

dove $\theta_{n}^{(r)}$ è l'angolo che da la direzione di $l_{n}^{(r)}$.

Per quanto si è dimostrato al $\mathrm{n}^{0} 29$, possiamo allora affermare che, comunque piccolo si fissi un intorno dei segmenti $l_{n}^{(r)}$, è sempre possibile costruire per ciascuno di essi una poligonale $p_{n}^{(r)}$, avente gli estremi comuni con $l_{n}^{(r)}$, appartenente ordinatamente all'intorno fissato e soddisfacente alla disuguaglianza

$$
\int_{p_{n}^{(r)}} F d \sigma^{\prime}-\int_{l_{n}^{(r)}} F d \sigma<-\frac{n l_{n}^{(r)}(\mathrm{I}-\cos \alpha)}{2}
$$

dove con $d \sigma$ e $d \sigma^{\prime}$ si indicano gli elementi d'arco di $l_{n}^{(r)}$ e $p_{n}^{(r)}$, rispettivamente.

$\left.3^{\mathbf{I}}\right)$ Cfr. loc. cit. $\left.{ }^{30}\right)$. 
Considerando perciò la poligonale $\vec{\Pi}_{n}$ che coincide con $\Pi_{n}$ fuori dei segmenti $l_{u}^{(r)}$ e che viene completata dalle poligonali $p_{n}^{(r)}$, abbiamo, indicando con $d \bar{s}_{n}$ il suo elemento d'arco

$$
\int_{\bar{\Pi}_{n}} F d \bar{s}_{n}-\int_{\Pi_{n}} F d s_{n}<-\frac{n \mu(\mathrm{I}-\cos \alpha)}{2},
$$

perchè la somma delle lunghezze dei segmenti $l_{n}^{(r)}$, che ricoprono interamente l'insieme $E_{n}^{\prime}$, è certamente non inferiore alla misura di tale insieme. Scelto dunque il numero \&, introdotto più sopra, minore di $\frac{n \mu(I-\cos \alpha)}{4}$, abbiamo, per la condizione $2^{2}$,

$$
\int_{\Pi_{n}} F d \bar{s}_{n}-\int_{C_{0}} F d s<-\frac{n \mu(1-\cos \alpha)}{4} .
$$

Siccome $\overline{\mathbf{\Pi}}_{n}$ puó esser scelto in modo da appartenere ad un intorno di $\mathbf{\Pi}_{n}$ prefissato ad arbitrio e $\Pi_{n}$, a sua volta, per $n$ sufficientemente grande, appartiene ad un intorno qualsiasi di $C_{0}$, abbiamo che, comunque si fissi un intorno di $C_{0}$, possiamo sempre costruire una poligonale $\overline{\boldsymbol{\Pi}}_{n}$ che appartenga ordinatamente ad esso e renda soddisfatta l'ultima disuguaglianza scritta. Ciò dimostra la nostra proposizione.

Osservazione. - Vale qui quanto si è detto nell'osservazione posta in fine al $\mathrm{n}^{\circ}$ precedente purchè, se non sono soddisfatte le condizioni $\mathrm{I}^{\circ}$ ) e $2^{\circ}$ ) ivi indicate, la curva $C_{o}$ abbia al più sulla frontiera del campo un insieme di punti di misura (contata sulla curva stessa) nulla.

34. Alla condizione espressa dal teorema del $\mathrm{n}^{0}$ precedente vogliamo aggiungerne un'altra.

Consideriamo un punto $P_{0}\left(x_{o}, y_{0}\right)$ interno al campo $A$ e supponiamo che esistano due angoli $\theta_{0}$ e $\bar{\theta}$ tali da rendere soddisfatta la disuguaglianza

$$
E\left(x_{0}, y_{0} ; \cos \theta_{0}, \operatorname{sen} \theta_{0} ; \cos \bar{\theta}, \operatorname{sen} \bar{\theta}\right)<0
$$

dove $E$ è la funzione, già definita al $\mathrm{n}^{\circ} 27$,

$$
E\left(x_{0}, y_{0} ; \cos \theta_{0}, \operatorname{sen} \theta_{0} ; \cos \bar{\theta}, \operatorname{sen} \bar{\theta}\right)
$$

$\equiv F\left(x_{0}, y_{0}, \cos \bar{\theta}, \operatorname{sen} \bar{\theta}\right)-\left\{\cos \bar{\theta} F_{x^{\prime}}\left(x_{0}, y_{0}, \cos \bar{\theta}_{o}, \operatorname{sen} \theta_{0}\right)+\operatorname{sen} \bar{\theta} F_{y^{\prime}}\left(x_{0}, y_{0}, \cos \theta_{0}, \operatorname{sen} \theta_{0}\right)\right\}$.

Per la continuita di questa funzione rispetto alle variabili da cui dipende, preso un numero positivo $n$, soddisfacente alla disuguaglianza

$$
E\left(x_{0} ; y_{0} ; \cos \theta_{0}, \operatorname{sen} \theta_{0} ; \cos \bar{\theta}, \operatorname{sen} \bar{\theta}\right)<-n,
$$

è possibile determinarne un altro $\delta$, pure positivo, tale che tutti $\mathrm{i}$ punti $(x y)$ che soddisfano alle condizioni

$$
\left|x-x_{\mathrm{o}}\right| \leq \delta, \quad\left|y-y_{\mathrm{o}}\right| \leq \delta
$$


risultino interni al campo $A$ e verifichino la disuguaglianza

$$
E\left(x, y ; \cos \theta_{0}, \operatorname{sen} \theta_{0} ; \cos \bar{\theta}, \operatorname{sen} \bar{\theta}\right)<-n .
$$

Premesso ció, conduciamo per $P_{\mathrm{o}}$ un segmento rettilineo $P_{\mathrm{o}} P_{\mathrm{I}}$, di lunghezza $l<\frac{\delta}{2}$, che faccia con la direzione positiva dell'asse delle $x$ l'angolo $\theta_{0}$, e dividiamolo in $n$ parti uguali mediante $\mathrm{i}$ punti

$$
P_{n, 0} \equiv P_{o}, \quad P_{n, 1}, \quad P_{n, 2}, \ldots, P_{n, n} \equiv P_{\mathrm{r}} .
$$

Per ciascun punto $P_{n, r}(r=0, \mathrm{I}, \ldots, n-\mathrm{I})$ conduciamo un segmento $P_{n, r} Q_{n, r}$ facente l'angolo $\bar{\theta}$ con la direzione positiva dell'asse delle $x$ e di lunghezza $l_{n}^{\prime}$ minore di $\frac{l}{2 n}$ ed anche di $\frac{n l}{12 n M}$, dove con $M$ abbiamo indicato il massimo modulo di $F_{1}(x, y, \cos \theta, \operatorname{sen} \theta)$ sul segmento $P_{0} P_{1}$, per tutti i possibili valori di $\theta$. Conduciamo poi gli $n$ segmenti $Q_{n, r} P_{n, r+1}$ e indichiamo con $C_{n}$ la spezzata $\left(P_{n 0} Q_{n 0} P_{n 1} Q_{n 1} \ldots P_{n n}\right)$ cosi risultante, spezzata che risulta completamente interna al campo $A$.

Facciamo corrispondere' ad ogni punto $N_{n r}$ del segmento $P_{n, r} Q_{n, r}$ il punto $N_{n, r}^{\prime}$ di $P_{n, r} P_{n, r+5}$, tale che la lunghezza di $P_{n r} N_{n r}^{\prime}$ sia uguale a quella di $P_{n r} N_{n r}$. Detto $Q_{n r}^{\prime}$ il punto corrispondente di $Q_{n r}$, facciamo corrispondere ad ogni punto $N_{n r}$ di $Q_{n r} P_{n, r+1}$ il punto $\mathrm{N}_{n r}^{\prime}$ di $Q_{n r}^{\prime} P_{n, r+r}$ tale che il rapporto della lunghezza di $Q_{n r} N_{n r}$ e $Q_{n r}^{\prime} N_{n r}^{\prime}$ sia uguale a quello della lunghezza di $Q_{n r} P_{n, r+1}$ e $Q_{n r}^{\prime} P_{n, r+1}$. Questo rapporto, che indicheremo con $R$, risulta compreso fra I e $\frac{\frac{\mathrm{I}}{n} l+l_{n}^{\prime}}{\frac{\mathrm{I}}{n} l-l_{n}^{\prime}}$ ossia fra I e 3 .

Scegliamo la lunghezza $s$ del segmento $P_{0} N_{n r}^{\prime}$ come parametro di una rappresentazione analitica simultanea del segmento $P_{0} P_{1}$ e della spezzata $C_{n}$, la quale conservi la corrispondenza ora indicata. Avremo, per $P_{0} P_{x}$, le equazioni

e per $C_{n}$,

$$
\begin{array}{lll}
x=x(s), & y=y(s), & (0 \leqslant s \leqslant l) \\
x=x_{n}(s), & y=y_{n}(s), & (0 \leq s \leq l) ;
\end{array}
$$

ed avremo anche, indicando con $s_{n}$ la lunghezza dell'arco di $C_{n}$ corrispondente all'intervallo $(0, s)$

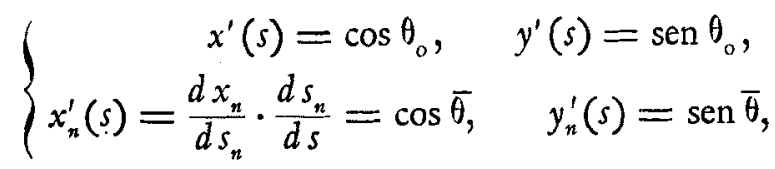

se il punto di $C_{n}$ corrispondente a $s$ appartiene ad un segmento $P_{n, r} Q_{n, r}$ e, in caso contrario,

$$
x_{n}^{\prime}(s)=R \cos \bar{\theta}^{\prime}, \quad y_{n}^{\prime}(s)=R \operatorname{sen} \bar{\theta}^{\prime}
$$


dove con $\theta^{\prime}$ indichiamo l'angolo che $Q_{n r} P_{n, r+1}$ forma con la direzione positiva dell'asse delle $x$. Osserviamo, prima di proseguire, che, per la costruzione fatta, risulta $\left|\bar{\theta}^{\prime}-\theta_{0}\right|<\frac{\pi}{2}$.

Consideriamo ora la differenza

$$
\Delta I=\int_{C_{n}} F d s_{n}-\int_{P_{0} P_{I}} F d s=\int_{0}^{i}\left\{F\left(x_{n}, y_{n}, x_{n}^{\prime}, y_{n}^{\prime}\right)-F\left(x, y, x^{\prime}, y^{\prime}\right)\right\} d s .
$$

Essa puó scriversi

$$
\Delta I=\int_{0}^{l}\left\{F\left(x_{n} y_{n} x_{n}^{\prime} y_{n}^{\prime}\right)-F\left(x y x_{n}^{\prime} y_{n}^{\prime}\right)\right\} d s+\int_{0}^{l}\left\{F\left(x y x_{n}^{\prime} y_{n}^{\prime}\right)-F\left(x y x^{\prime} y^{\prime}\right)\right\} d s,
$$

ed anche, ricordando l'omogeneità della $F\left(x y x^{\prime} y^{\prime}\right)$ rispetto a $x^{\prime}, y^{\prime}$ e la definizione della funzione $E$,

(29)

$$
\left\{\begin{array}{c}
\Delta I=\int_{0}^{l}\left\{F\left(x_{n} y_{n} x_{n}^{\prime} y_{n}^{\prime}\right)-F\left(x y x_{n}^{\prime} y_{n}^{\prime}\right)\right\} d s \\
+\int_{0}^{l}\left\{\left(x_{n}^{\prime}-x^{\prime}\right) F_{x^{\prime}}\left(x y x^{\prime} y^{\prime}\right)+\left(y_{n}^{\prime}-y^{\prime}\right) F_{y^{\prime}}\left(x y x^{\prime} y^{\prime}\right)\right\} d s \\
\quad+\int_{0}^{l} E\left(x y ; x^{\prime}, y^{\prime} ; x_{n}^{\prime}, y_{n}^{\prime}\right) d s .
\end{array}\right.
$$

Indicando con $s_{n, r}$ la lunghezza del segmento $P_{o} P_{n r}$, e con $s_{n, r}^{\prime}$ quella di $P_{0} Q_{n r}^{\prime}$, possiamo scrivere

$$
\begin{gathered}
\int_{0}^{l} E\left(x, y ; x^{\prime}, y^{\prime} ; x_{n}^{\prime}, y_{n}^{\prime}\right) d s=\sum_{r=0}^{n=-1} \int_{s_{n, r}}^{s_{n, r}^{\prime}} E\left(x, y ; x^{\prime}, y^{\prime} ; x_{n}^{\prime}, y_{n}^{\prime}\right) d s \\
+\sum_{r=0}^{n-1} \int_{s_{n, r}^{\prime}}^{s_{n, r+1}} E\left(x, y ; x^{\prime}, y^{\prime} ; x_{n}^{\prime}, y_{n}^{\prime}\right) d s,
\end{gathered}
$$

e rammentando le $(28)$ e $\left(28^{\prime}\right)$,

$$
\begin{gathered}
=\sum_{r=0}^{n-I} \int_{s_{n r}}^{s_{n r}^{\prime}} E\left(x, y, \cos \theta_{0}, \operatorname{sen} \theta_{0} ; \cos \bar{\theta}, \operatorname{sen} \bar{\theta}\right) d s \\
+R \sum_{r=0}^{n=1} \int_{s_{n r}^{\prime}}^{s_{n, r+1}} E\left(x, y ; \cos \theta_{0}, \operatorname{sen} \theta_{0} ; \cos \overline{\theta^{\prime}}, \operatorname{sen} \overline{\theta^{\prime}}\right) d s,
\end{gathered}
$$

ed anche per la (27) e per essere $R<3$,

$$
\begin{gathered}
\int_{0}^{l} E\left(x, y ; x^{\prime}, y^{\prime} ; x_{n}^{\prime}, y_{n}^{\prime}\right) d s<-n n l_{n}^{\prime} \\
+3 \sum_{r=0}^{n=1} \int_{s_{n, r}^{\prime}}^{s_{n, r+1}} E\left(x, y ; \cos \theta_{o}, \operatorname{sen} \theta_{0} ; \cos \overline{\theta^{\prime}}, \operatorname{sen} \overline{\theta^{\prime}}\right) d s .
\end{gathered}
$$

Rend. Circ. Matem, Palermo, t. XLIV (1920). - Stampato it it settembre igzo. 
Inoltre, dalla formula stabilita al $n^{\circ} 9$, si ricava

$$
E\left(x, y, \cos \theta_{0}, \operatorname{sen} \theta_{0} ; \cos \bar{\theta}^{\prime}, \operatorname{sen} \bar{\theta}^{\prime}\right)
$$

$=F\left(x, y, \cos \bar{\theta}^{\prime}, \operatorname{sen} \bar{\theta}^{\prime}\right)-\left\{\cos \bar{\theta}^{\prime} F_{x^{\prime}}\left(x, y, \cos \theta_{0}, \operatorname{sen} \theta_{0}\right)+\operatorname{sen} \bar{\theta}^{\prime} F_{y^{\prime}}\left(x, y, \cos \theta_{0}, \operatorname{sen} \theta_{0}\right)\right.$

$$
=\int_{\theta_{0}}^{\bar{\theta} \prime} F_{\mathrm{I}}(x, y, \cos \alpha, \operatorname{sen} \alpha) \sin \left(\overline{\theta^{\prime}}-\alpha\right) d \alpha
$$

e per essere $\left|F_{\mathrm{r}}(x, y, \cos \alpha, \operatorname{sen} \alpha)\right| \leqslant M$,

$$
E\left(x, y ; \cos \theta_{0}, \operatorname{sen} \theta_{0} ; \cos \bar{\theta}^{\prime}, \operatorname{sen} \bar{\theta}^{\prime}\right) \leq M\left(I-\cos \left(\bar{\theta}^{\prime}-\theta_{0}\right)\right) .
$$

Ma dal triangolo $P_{n r} Q_{n r} P_{n, r+1}$ si trae, per la relazione dei seni,

$$
\frac{l_{n}^{\prime}}{\operatorname{sen}\left|\bar{\theta}^{\prime}-\theta_{0}\right|}=\frac{l_{n}^{\prime \prime}}{\operatorname{sen}\left|\bar{\theta}-\theta_{0}\right|},
$$

dove si è indicato con $l_{*}^{\prime \prime}$ la lunghezza del lato $Q_{n r} P_{n r+1}$, e perció

$$
\left.\operatorname{sen}^{2} \overline{\theta^{\prime}}-\theta_{0}\right)<\left(\frac{l_{n}^{\prime}}{\frac{l}{n}-l_{n}^{\prime}}\right)^{2},
$$

essendo $l_{n}^{\prime \prime}>\frac{\mathrm{I}}{n} l-l_{n}^{\prime}$. Si ha dunque, per essere, come gid abbiamo osservato, $\left|\bar{\theta}^{\prime}-\theta_{0}\right|<\frac{\pi}{2}$ e quindi

$$
\begin{gathered}
\left.\frac{\mathrm{I}-\cos \left(\bar{\theta}^{\prime}-\theta_{0}\right)}{\operatorname{sen}^{2}\left(\overline{\theta^{\prime}}-\theta_{0}\right)}<\mathrm{I}^{32}\right), \\
\mathrm{I}-\cos \left(\bar{\theta}^{\prime}-\theta_{0}\right)<\left(\frac{l_{n}^{\prime}}{\frac{l}{n}-l_{n}^{\prime}}\right)^{2} .
\end{gathered}
$$

$$
\begin{gathered}
E\left(x, y ; \cos \theta_{0}, \operatorname{sen} \theta_{0} ; \cos \bar{\theta}^{\prime}, \operatorname{sen} \bar{\theta}^{\prime}\right)<M\left(\frac{l_{n}^{\prime}}{\frac{l}{n}-l_{n}^{\prime}}\right)^{2}, \\
\int_{0}^{l} E\left(x, y ; x^{\prime}, y^{\prime} ; x_{n}^{\prime}, y_{n}^{\prime}\right) d s<-n n l_{n}^{\prime}+3 n\left(\frac{l}{n}-l_{n}^{\prime}\right) M\left(\frac{l_{n}^{\prime}}{\frac{l}{n}-l_{n}^{\prime}}\right)^{2} .
\end{gathered}
$$

32) Posto, infatti,

$$
y=\frac{1-\cos x}{\operatorname{sen}^{2} x}
$$

$y^{\prime}>0$ per $0<x \leqslant \frac{\pi}{2}$, c $y=\mathrm{I}$ per $x=\frac{\pi}{2}$. 
Essendo poi $l_{n}^{\prime}<\frac{l}{2 n}$, il secondo membro di questa disuguaglianza è minore di

$$
n l_{n}^{\prime}\left(-n+\frac{6 M n l_{n}^{\prime}}{l}\right)
$$

ed essendo anche $l_{n}^{\prime}<\frac{n l}{12 n M}$, questa espressione è minore di

$$
n l_{n}^{\prime}\left(-n+\frac{n}{2}\right)<-\frac{n l \lambda}{2}
$$

dove abbiamo indicato con $\lambda$ il valore del rapporto $\frac{l_{n}^{\prime}}{\frac{I}{n} l}$.

E dunque

$$
\int_{0}^{1} E\left(x, y ; x^{\prime}, y^{\prime} ; x_{n}^{\prime}, y_{n}^{\prime}\right) d s<-\frac{n l \lambda}{2} .
$$

Circa gli altri integrali che figurano nel secondo membro di (29), possiamo affermare che, per $n \rightarrow \infty$, tendendo $x_{n}$ e $y_{n}$ uniformemente, su tutto l'intervallo $(0, l)$, a $x(s)$ e $y(s)$, rispettivamente, ed essendo sempre $\mathbf{I} \leqslant x_{n}^{\prime 2}+y_{n}^{\prime 2}<3$, per la continuità della funzione $F$ l'integrale

$$
\int_{0}^{\prime}\left\{F\left(x_{n}, y_{n}, x_{n}^{\prime}, y_{n}^{\prime}\right)-F\left(x, y, x_{n}^{\prime}, y_{n}^{\prime}\right)\right\} d s
$$

tende a zero e che a zero tende anche

$$
\int_{0}^{l}\left\{\left(x_{n}^{\prime}-x^{\prime}\right) F_{x^{\prime}}\left(x, y, x^{\prime}, y^{\prime}\right)+\left(y_{n}^{\prime}-y^{\prime}\right) F_{y^{\prime}}\left(x, y, x^{\prime}, y^{\prime}\right)\right\} d s,
$$

in forza del lemma del $\mathrm{n}^{\circ} 8$.

Resta cosi provato che, per ogni $n$ maggiore di un certo $\bar{n}$, ’

$$
\Delta I<-\frac{n l \lambda}{4}
$$

e siccome, preso comunque un numero $\rho>0$, si puó sempre trovare un $n$ per il quale la spezzata $C_{n}$ appartenga ordinatamente allintorno $(\rho)$ del segmento $P_{0} P_{x}$, resta anche provato che su questo segmento lintegrale della $F$ non puo esser funzione semicontinua inferiormente, se è verificata l'ipotesi formulata al principio di questo $\mathrm{n}^{\mathbf{0}}$.

35. Dal risultato ottenuto al $\mathrm{n}^{\circ}$ precedente, ragionando in modo perfettàmente analogo a quanto si è fatto ai $\mathrm{n}^{\mathrm{i}} 32$ e 33 , deduciamo le due proposizioni che seguono:

a) Condizioni necessaria affinche su una data curva $C_{\mathrm{o}}$, completamente interna al campo $A$ e dotata ovunque di tangente variabile in modo continuo, lintegrale $I_{C}$ sia funzione semicantinua inferiormente, $\dot{e}$ che in ogni punto $\left(x_{0} y_{0}\right)$ della curva si abbia, per 
tutti i possibili valori di $\theta$,

$$
E\left(x_{0}, y_{0} ; \cos \theta_{0}, \operatorname{sen} \theta_{0} ; \cos \theta, \operatorname{sen} \theta\right) \gtrsim 0,
$$

dove $\theta_{0}$ indica langolo che la direzione positiva della tangente alla curva stissa forma con la direzione positiva dell'asse delle $x$.

b) Condizione necessaria affincbe, su una data curva $C_{0}$, completamente intorna al campo $A$, l'integrale $I_{C}$ sia funzione semicontinua inferiormente, è che abbia misura nulla l'insieme dei punti $\left(x_{0} y_{0}\right)$ di $C_{0}$ nei quali non è verificata, per tutti $i$ possibili valori di $\theta$, la disuguaglianza

$$
E\left(x_{0}, y_{0} ; \cos \theta_{0}, \operatorname{sen} \theta_{0} ; \cos \theta, \operatorname{sen} \theta\right) \gg 0,
$$

dove $\theta_{0}$ indica l'angolo che la direzione positiva della tangente alla curva stessa, là dove questa tangente esiste, forma con la direzione positiva dell'asse $x$.

Osservazione. - Quanto si è detto nelle Osservazioni poste in fine ai $\mathrm{n}^{\mathrm{i}} 32$ e 33 puó ripetersi per le precedenti proposizioni $a$ ) e $b$ ).

Capitolo II.

GLI INTEGRALI PER LE CURVE IN FORMA NON PARAMETRICA.

$\oint \mathrm{I}$.

\section{Definizioni.}

36. Considereremo sempre, nel seguito, curve $C$ del piano $(x y)$ definite da un'equazione

$$
y=y(x)
$$

dove la $x$ assume tutti $\mathrm{i}$ valori di un intervallo $(a, b)$, non necessariamente lo stesso per tutte le curve, e la $y(x)$ è una funzione assolutamente continua in tutto l'intervallo indicato.

Rammentiamo che una funzione $y(x)$ dicesi assolutamente continua ${ }^{33}$ ) in un in-

33) Cfr. G. Vitali, Sulle funzioni integrali [Atti della R. Accademia delle Scienze di Torino, Vol. XL (I904-I905), pp. I021-1034]. 
tervallo $(a, b)$ se, preso un numero positivo $\sigma$ qualunque, si puó poi sempre determinarne un altro $\mu>0$ tale che sia

$$
\left|\sum_{i}\left\{y\left(\beta_{i}\right)-y\left(\alpha_{i}\right)\right\}\right|<\sigma
$$

dove la sommatoria è estesa ad un qualsiasi gruppo di intervalli $\left(\alpha_{i}, \beta_{i}\right)$ di $(a, b)$, non sovrapponentisi e aventi una somma totale minore di $\mu$. Rammentiamo, inoltre, che se $y(x)$ è funzione assolutamente continua in $(a, b)$, in questo intervallo esiste quasi ovunque la derivata $y^{\prime}(x)$ finita ed è

$$
y\left(x_{2}\right)-y\left(x_{1}\right)=\int_{x_{1}}^{x_{2}} y^{\prime}(x) d x
$$

per due qualunque punti $x_{1}$ e $x_{2}$ di $(a, b)$. Reciprocamente, ogni funzione integrale è assolutamente continua.

37. Diremo che una curva

C:

$$
y=y(x)
$$

appartiene all'intorno $(p)$ della curva $C_{\mathrm{o}}$ :

$$
y=y_{0}(x)
$$$$
\left(a_{0} \leq x \leq b_{0}\right)
$$

se, in ogni punto $x$ comune ai due intervalli $\left(a_{0}, b_{0}\right),(a, b)$, è

$$
\left|y(x)-y_{0}(x)\right|<\rho,
$$

e se, inoltre, i punti che appartengono all'uno o all'altro dei due intervalli, senza appartenere ad entrambi, costituiscono dei segmenti (due al massimo) di lunghezza minore di $\rho$, e ciascuno degli archi delle curve $C_{\circ}$ e $C$ che corrispondono a questi segmenti è interno al cerchio, di raggio $p$, avente il centro nell'estremo corrispondente di $C_{0}$.

38. Sia $A$ un campo (insieme di punti), del piano ( $x y$ ) tale che:

$\left.I^{\circ}\right)$ i suoi punti appartenenti ad un qualsiasi cerchio dello stesso piano costituiscano sempre un insieme chiuso;

$\left.2^{\circ}\right)$ considerata una qualunque curva $C_{0}\left(\mathrm{n}^{\circ} 36\right)$ costituita tutta di punti di $A$, si possa sempre trovare un numero positivo $\rho$ in guisa che, se $C$ è un'altra qualsiasi curva ( $\left.\mathrm{n}^{\circ} 36\right)$, anch'essa costituita tutta di punti di $A$ e appartenente all'intorno ( $p$ ) della $C$, facciano parte del campo $A$ tutti i punti del piano $(x y)$ le cui coordinate soddisfino alle disuguaglianze

$$
\begin{gathered}
a_{0} \leq x \leq b_{0}, \\
a \leq x \leq b, \\
y_{0}(x) \leqq y \leqq y(x)
\end{gathered}
$$


intendendo che nell'ultima figuri il segno di minore o di maggiore secondo che è $y_{0}(x)$ minore o maggiore di $y(x)$.

39. Sia $f\left(x, y, y^{\prime}\right)$ una funzione, definita per ogni punto $(x y)$ del campo $A$ e per ogni valore finito di $y^{\prime}$, e soddisfacente alle seguenti condizioni:

$\left.I^{\circ}\right)$ sia continua in ogni punto $\left(x, y, y^{\prime}\right)$ in cui è definita;

$2^{\circ}$ ) ammetta, in tutti gli stessi punti, le derivate parziali $f_{y^{\prime}}, f_{y^{\prime} y^{\prime}}, f_{y^{\prime} x}$, e queste derivate risultino continue.

40. Considerata una curva $C\left(\mathrm{n}^{\circ} 36\right)$ del campo $A$ per la quale esista l'integrale (nel senso del Lebesgue)

$$
\int_{a}^{b} f\left(x, y(x), y^{\prime}(x)\right) d x
$$

si indicherd brevemente quest'integrale con la scrittura

ed anche con $I_{C}$.

$$
\int_{C} f d x
$$

L'integrale $I_{\mathcal{C}}$ risulta evidentemente una funzione della curva $C$, definita per ogni curva del $n^{\circ} 36$, appartenente al campo $A$, per la quale l'integrale stesso esista.

Nel seguito considereremo soltanto curve che soddisfino alle condizioni ora indicate.

Per semplicità di ragionamento, supporremo poi sempre che gli intervalli su cui sono definite le funzioni $y(x)$, relative alle nostre curve, siano tutte fra loro coincidenti.

I risultati che otterremo al $\ 5$ valgono peraltro indipendentemente da questa ipotesi e cioè per tutte le curve $C$ sopra definite; altrettanto puó dirsi per quelli dei $\$ \subseteq 2$, 3,4 , purchè peró gli estremi delle curve considerate siano interni al campo $A$. È facile vedere che, per poter togliere la restrizione relativa agli estremi, basta:

a) che la funzione $f\left(x, y, y^{\prime}\right)$ possa definirsi anche fuori del proprio campo, in un campo $A^{\prime}$ che contenga come punti interni quelli di $A$, e in modo da ammettere, anche nei nuovi punti, compresa la frontiera di $A$, le proprieta enunciate al $\mathbf{n}^{\circ} 39$;

$b$ ) oppure, che la parte della frontiera di $A$ contenuta in ogni porzione limitata di piano sia costituita di un numero finito di curve continue, senza punti multipli, aventi due a due al più un numero finito di punti comuni, ciascuna delle quali risulti composta di un numero finito di archi a tangente variabile in modo continuo;

c) oppure, che la $f$ resti sempre maggiore o sempre minore di un numero fisso;

d) oppure, che in nessun punto della frontiera di $A$ sia $f_{y^{\prime} y^{\prime}}\left(x, y y^{\prime}\right)=0$ per tutti i valori di $y^{\prime}$.

4I. Diremo che $I_{C}$ è una funzione semicontinua inferiormente (superiormente) sulla curva $C_{o}$ se, preso un numero positivo $\varepsilon$, ad arbitrio, è sempre possibile di determinarne un altro, pure positivo, $\rho$ tale che la disuguaglianza

$$
I_{C}>I_{C_{0}}-\varepsilon, \quad\left(<I_{C_{0}}+\varepsilon\right)
$$


sia verificata da tutte le curve che appartengono all'intorno ( $\rho$ ) di $C_{0}$. Qualora sia invece sempre verificata la disuguaglianza

$$
\left|I_{C}-I_{C_{0}}\right|<\varepsilon
$$

la $I_{C}$ sard detta funzione continua sulla curva $C_{0}$.

42. Diremo, infine, che l'integrale $I_{C}$ è regolare se, in ogni punto del campo $A$ e per ogni $y^{\prime}$ finito, la derivata $f_{y^{\prime} y^{\prime}}$ conserva sempre uno stesso segno, senza mai annullarsi; quando invece, pur conservando sempre lo stesso segno, la $f_{y^{\prime} y^{\prime}}$ puó annullarsi, l'integrale $I_{C}$ lo diremo quasi-regolare.

\section{$\int 2$.}

\section{Condizioni sufficienti per la semicontinuità.}

a) II caso lineare.

43. Considereremo qui il caso più semplice, quello cioè in cui la funzione $f\left(x, y, y^{\prime}\right)$ è lineare rispetto alla $y^{\prime}$. Supporremo dunque

$$
f\left(x, y, y^{\prime}\right) \equiv P(x, y)+Q(x, y) y^{\prime} .
$$

Per le ipotesi fatte al $\mathrm{n}^{\circ} 39$, le funzioni $P(x, y), Q(x, y)$ saranno funzioni continue in tutto il campo $A$, nel quale esisterd pure e sard continua la derivata parziale $\frac{\partial Q}{\partial x}$. Nel caso attuale abbiamo evidentemente, $f_{y^{\prime} y^{\prime}} \equiv \mathrm{o}$ per tutte le terne $\left(x, y, y^{\prime}\right)$ ammesse: abbiamo anche che l'esistenza dell'integrale $I_{C}$ è conseguenza immediata dell'assoluta continuità delle funzioni $y(x)$. zioni

Siano $C_{\mathrm{o}}$ e $C$ due curve di $A\left(\mathrm{n}^{\circ} 4^{0}\right)$, rappresentate rispettivamente dalle equa-

$$
\begin{aligned}
& y=y_{0}(x), \\
& y=y(x),
\end{aligned}
$$

sull'intervallo comune $(a, b)$, e esaminiamo la differenza $I_{C}-I_{C_{0}}$. Essa puó scriversi

$$
\int_{a}^{b}\left\{P(x, y(x))-P\left(x, y_{\circ}(x)\right)\right\} d x+\int_{C} Q y^{\prime} d x-\int_{C_{0}} Q y^{\prime} d x .
$$

Il primo integrale, per la continuità della funzione $P(x, y)$, puó rendersi piccolo a piacere col prendere convenientemente piccolo lintorno di $C_{\mathrm{o}}$ cui appartiene la $C$; vale a dire, preso ad arbitrio un $\varepsilon>0$, possiamo determinare un $\rho_{\mathrm{r}}>0$ tale che, per ogni curva $C$ appartenente allintorno $\left(p_{1}\right)$ della $C_{\rho}$, si abbia

$$
\left|\int_{a}^{b}\left\{P(x, y(x))-P\left(x, y_{0}(x)\right)\right\} d x\right|<\frac{\varepsilon}{2} .
$$


Studiamo ora la differenza

che possiamo scrivere

$$
\int_{C} Q y^{\prime} d x-\int_{C_{o}} Q y^{\prime} d x
$$

$$
\int_{C} Q d y-\int_{C_{0}} Q d y
$$

Indichiamo con $\gamma$ la curva composta del segmento rettilineo avente per estremi, punti $\left(a, y_{\mathrm{o}}(a)\right),(a, y(a))$, della curva $C$, del segmento rettilineo avente per estremi i punti $(b, y(b)),\left(b, y_{o}(b)\right)$, e della curva $C_{o}$ percorsa in senso negativo, cioè secondo le $x$ decrescenti. Abbiamo

$$
\begin{gathered}
\int_{C} Q d y-\int_{C_{0}} Q d y=\int_{Y} Q d y-\int_{y_{0}(a)}^{y(a)} Q(a, y) d y-\int_{y(b)}^{y_{0}(b)} Q(b, y) d y \\
=\int_{Y} Q d y-\left\{y(a)-y_{0}(a)\right\} Q(a, \bar{y})-\left\{y_{0}(b)-y(b)\right\} Q(b, \bar{y}),
\end{gathered}
$$

dove $\bar{y}$ e $\bar{y}$ indicano due valori di $y$ compresi fra $y_{0}(a)$ e $y(a)$ e fra $y(b)$ e $y_{0}(b)$, rispettivamente. Se con $M$ rappresentiamo il massimo modulo di $Q$ in un campo limitato che contenga la curva $C_{0}$ e tutte quelle che appartengono all'intorno $\left(p_{1}\right)$ di essa, abbiamo

$$
\int_{C} Q d y-\int_{C_{\mathrm{o}}} Q d y=\int_{r} Q d y-2 \rho_{\mathrm{I}} M \theta
$$

con $-\mathrm{I} \leqslant \theta \leqslant \mathrm{I}$.

Per la formula di Green, se $\Gamma$ indica il campo racchiuso dalla curva $\gamma^{34}$ ), è

$$
\left|\int_{\curlyvee} Q d y\right| \leqslant \int_{\Gamma}\left|\frac{\partial Q}{\partial x}\right| d \Gamma
$$

e siccome l'area di $\Gamma$ è minore di $p_{1}(b-a)$, detto $M_{\mathrm{r}}$ il massimo modulo di $\frac{\partial Q}{\partial x}$ nel campo limitato sopra indicato, è anche

e percio

$$
\left|\int_{Y} Q d y\right|<M_{s} p_{s}(b-a)
$$

$$
\left|\int_{C} Q d y-\int_{C_{0}} Q d y\right|<\rho_{1}\left(M_{\mathrm{r}}(b-a)+2 M\right) \text {. }
$$

34) Un punto $\overline{(x}, \bar{y})$ del piano $(x, y)$ appartiene a $\Gamma$ se è solamente se è $a \leqslant \bar{x} \leqslant b$ e $\bar{y}$ ̀̀ compreso fra $y_{0}(\bar{x})$ e $y(x)$, estremi inclusi. 
Da questa disuguaglianza e dalla (30) deduciamo

$$
\left|I_{C}-I_{C_{\mathrm{o}}}\right|<\varepsilon
$$

per ogni curva $C$ appartenente all'intorno $(\rho)$ della $C_{0}$, dove $\rho$ è $<p_{1}$ ed anche minore di $\frac{\varepsilon}{2\left\{M_{1}(b-a)+2 M\right\}}$. Ció dimostra il seguente teorema :

L'integrale

$$
\int_{C}\left\{P(x, y)+Q(x, y) y^{\prime}\right\} d x
$$

dove $P$ e $Q$ sono funzioni continue nel campo $A$ insieme con la derivata $\frac{\partial Q}{\partial x}$, $\dot{e}$ funzione continua della curva $C\left(\mathrm{n}^{\circ} 40\right)$.

44. Osservazione I. - Nel capitolo precedente, al $n^{\circ} 3 \mathrm{I}$ abbiamo veduto che se si considerano le curve in forma parametrica, se cioè non si impone la condizione che ogni curva sia incontrata in un sol punto al più da una qualsiasi parallela all'asse delle $y$, l'integrale

$$
\int_{C} P d x+Q d y
$$

non è sempre una funzione continua della curva d'integrazione, pur essendo soddisfatte tutte le possibili condizioni di continuità e derivabilità relative alle $P$ e $Q$. La differenza fra $i$ due casi sta in questo che, per la condizione, indicata or ora, a cui soddisfano le curve che si considerano in questo capitolo, l'area $\Gamma$ tende a zero con $\rho$, il che invece non accade quando la condizione detta non si pone e si consente perciò alle curve di compiere infiniti giri intorno ad un punto e quindi all'area $\Gamma$ di ricoprire infinite volte la stessa porzione di piano.

Osservazione II. - E facile verificare che, per la validita del teorema del $n^{0}$ precedente, è sufficiente supporre che le $P$ e $Q$ siano funzione continue nel campo $A$ e che, inoltre, la $Q$, come funzione della $x$, risulti assolutamente continua ed abbia la derivata parziale $\frac{\partial Q}{\partial x}$ (che per l'assoluta continuità della $Q$ esiste quasi dappertutto) superficialmente integrabile.

45. E interessante rilevare che la continuità dell'integrale dell'espressione $P+Q y^{\prime}$ puó mancare pur essendo le funzioni $P$ e $Q$ continue. Consideriamo infatti il seguente esempio.

Fissiamo, sul segmento $(\mathrm{o}, \mathrm{I})$ dell'asse della $x$, l'intervallo $\left(\frac{\mathrm{I}}{2 n}, \frac{\mathrm{I}}{2 n-\mathrm{I}}\right)$ $(n=\mathrm{I}, 2, \ldots)$ e dividiamolo in tre parti uguali mediante $\mathrm{i}$ punti

$$
\alpha_{2 n}=\frac{\mathrm{I}}{2 n}+\frac{\mathrm{I}}{6 n(2 n-\mathrm{I})}, \quad \beta_{2 n}=\frac{\mathrm{I}}{2 n}+\frac{\mathrm{I}}{3 n(2 n-\mathrm{I})},
$$

Rend. Circ. Matem. Palermo, t. XLIV (1920). - Stampato il 18 settembre 1920. 
e poniamo

$$
\begin{aligned}
& Q(x, y)=Q(x) \\
& Q\left(\frac{\mathrm{I}}{2 n}\right)=Q\left(\frac{\mathrm{I}}{2 n-\mathrm{I}}\right)=0 \\
& Q\left(\alpha_{2 n}\right)=Q\left(\beta_{2 n}\right)=\frac{\mathrm{I}}{\sqrt{2 n}},
\end{aligned}
$$

imponendo alla $Q(x)$ di avere in $\left(\frac{\mathrm{I}}{2 n}, \frac{\mathrm{I}}{2 n-\mathrm{I}}\right)$ un sol massimo e nessun minimo (relativo). Sard dunque sempre, in quest'intervallo, $Q(x) \gg 0$.

Dividiamo in tre parti uguali anche l'intervallo $\left(\frac{\mathrm{I}}{2 n+1}, \frac{\mathrm{I}}{2 n}\right)(n=1,2, \ldots)$, mediante $\mathrm{i}$ punti

$$
\alpha_{2 n+1}=\frac{I}{2 n+I}+\frac{I}{6 n(2 n+I)}, \quad \beta_{2 n+1}=\frac{I}{2 n+I}+\frac{I}{3 n(2 n+I)},
$$

e poniamo

$$
\begin{aligned}
Q(x y) & =Q(x), \\
Q\left(\frac{\mathrm{I}}{2 n+\mathrm{I}}\right) & =Q\left(\frac{\mathrm{I}}{2 n}\right)=0, \\
Q\left(\alpha_{2 n+1}\right) & =Q\left(\beta_{2 n+1}\right)=-\frac{\mathrm{I}}{\sqrt{2 n}+\mathrm{I}},
\end{aligned}
$$

imponendo alla $Q(x)$ di avere in $\left(\frac{I}{2 n+1}, \frac{I}{2 n}\right)$ un sol minimo e nessun massimo (relativo), il che porta che, in questo intervallo, sia sempre $Q(x) \leq 0$.

Poniamo, infine, $Q(0)=0$.

Definiamo ora una funzione $\varphi(x)$ nel seguente modo:

$$
\begin{aligned}
& \text { in }\left(\frac{1}{2 n}, \alpha_{2 n}\right) \quad \text { sia } \quad \varphi(x)=Q\left(\alpha_{2 n}\right) \text {, } \\
& \text { " }\left(\beta_{2 n}, \frac{\mathrm{I}}{2 n-\mathrm{I}}\right), \quad \varphi(x)=0 \text {, } \\
& \text { ) }\left(\alpha_{2 n}, \beta_{2 n}\right) \quad \text { la } \varphi(x) \text { sia lineare; } \\
& \text { " }\left(\frac{\mathrm{I}}{2 n+\mathrm{I}}, \alpha_{2 n+1}\right), \quad \varphi(x)=0 \\
& \text { ) } \quad \beta_{2 n+1} \quad \phi\left(\beta_{2 n+1}\right)=-Q\left(\beta_{2 n+1}\right) \\
& \text { ) }\left(\alpha_{2 n+1}, \beta_{2 n+1}\right) \text { la } \varphi(x) \text { sia lineare } \\
& \text { » }\left(\beta_{2 n+1}, \frac{I}{2 n}\right) \quad \text { la } \varphi(x) \text { sia lineare } \\
& \text { » } \quad \text { o } \quad \varphi(0)=0 \text {. }
\end{aligned}
$$


E ciò sia per ogni $n=\mathrm{I}, 2, \ldots$.

E evidentemente

$$
\begin{aligned}
& \int_{\frac{1}{2 n}}^{\frac{1}{2 n-1}} Q(x) \varphi^{\prime}(x) d x<-\frac{\mathrm{I}}{2 n}, \\
& \int_{\frac{1}{2 n+1}}^{\frac{I}{2 n}} Q(x) \varphi^{\prime}(x) d x<-\frac{\mathrm{I}}{2 n+\mathrm{I}},
\end{aligned}
$$

e perciò

$$
\lim _{x \rightarrow+0} \int_{x}^{1} Q(x) \varphi^{\prime}(x) d x \leq-\lim _{n \rightarrow \infty}\left(\frac{I}{2}+\frac{1}{3}+\frac{1}{4}+\cdots+\frac{1}{n}\right)=-\infty .
$$

Ció posto, definiamo la curva $C_{n}$, di equazione $y=y_{n}(x)$, ponendo

$$
\begin{array}{llrl}
y_{n}(x)=0 \quad \text { in } & \left(0, \frac{\mathrm{I}}{4 n+\mathrm{I}}\right), \\
y_{n}(x)=\varphi(x) \quad \text { " } & \left(\frac{\mathrm{I}}{4 n+\mathrm{I}}, \frac{\mathrm{I}}{2 n+\mathrm{I}}\right), \\
y_{n}(x)=0 \quad & \quad\left(\frac{\mathrm{I}}{2 n+\mathrm{I}}, \mathrm{I}\right) .
\end{array}
$$

$\mathrm{E}$ allora, in tutto (o, I),

$$
0 \leqslant y_{n}(x)<\frac{\mathrm{I}}{\sqrt{2 n}}
$$

e

$$
\begin{aligned}
\int_{0}^{1} Q(x) y_{n}^{\prime}(x) d x & <-\left(\frac{\mathrm{I}}{4 n+\mathrm{I}}+\frac{\mathrm{I}}{4 n}+\frac{\mathrm{I}}{4 n-\mathrm{I}}+\cdots+\frac{\mathrm{I}}{2(n+\mathrm{I})}\right) \\
& <-\frac{2}{5} .
\end{aligned}
$$

Questa disuguaglianza, unita alla (3I), mostra che sulla curva costituita dal segmento $(0, \mathrm{I})$ dell'asse delle $x$, per la quale è

$$
\int_{0}^{1} Q(x) y^{\prime}(x) d x=0,
$$

l'integrale dell'espressione $Q(x) y^{\prime}(x)$ non è funzione continua e neppure semicontinua inferiormente.

Altrettanto accade per lo stesso integrale, in cui sia

$$
\begin{array}{cccc}
Q(x y)=\sqrt{x} \sin \frac{1}{x} & \text { per } & x>0, \\
Q(x y)=0 & \text { per } & x & =0 .
\end{array}
$$


46. Quando non siano soddisfatte le condizioni dell'enunciato del $n^{\circ} 430$ dell'Osservazione II del $\mathrm{n}^{\circ} 44$, puó riuscire utile il seguente teorema:

" Se $P(x y)$ e $Q(x y)$ sono due funzioni continue in tutto il campo $A$, l'integrale

$$
\int_{C}\left\{P(x y)+Q(x y) y^{\prime}\right\} d x
$$

è funzione continua della curva d'integrazione nella classe delle curve $C\left(\mathrm{n}^{\circ} 4^{\circ}\right)$ per le quali si ba

$$
\int_{a}\left|y^{\prime}\right|^{1+\alpha} d x<M
$$

dove $\propto$ e $M$ sono due numeri fissi positivi o nulli.

Questa proposizione non è che un corollario di quella del $\mathrm{n}^{\circ} 25$.

$$
\$ 3 \text {. }
$$

\section{Condizioni sufficienti per la semicontinuità:}

b). Il caso quasi regolare.

47. Analogamente a quanto si è fatto nel capitolo precedente per le curve in forma parametrica, tratteremo anche qui soltanto il caso in cui sia

$$
f_{y^{\prime} y^{\prime}}\left(x, y, y^{\prime}\right) \supseteq 0
$$

per tutte le terne di valori $x, y, y^{\prime}$ che devonsi considerare, potendosi l'altro, in cui sia invece verificata sempre la disuguaglianza $f_{y^{\prime} y^{\prime}} \leqslant 0$, dedurre da questo cambiando la funzione $f\left(x, y, y^{\prime}\right)$ in $-f\left(x, y, y^{\prime}\right)$.

48. Cominciamo con il dimostrare che:

$L^{\prime}$ 'integrale quasi regolare $I_{C}$, con $f_{y^{\prime} y^{\prime}} \gg 0$, ¿ funzione semicontinua inferiormente su ogni curva $C_{0}$, di equazione $y=y_{0}(x)$, per la quale la $y_{0}(x)$ abbia numeri derivati costantemente inferiori ad un numero fisso.

La dimostrazione che ora daremo sard fondata sulla sostituzione della funzione $f\left(x, y, y^{\prime}\right)$ con un'altra funzione $E$ la quale:

$\left.r^{\circ}\right)$ differisca dalla $f$ per una funzione il cui integrale vari con continuità al variare della curva d'integrazione;

$2^{\circ}$ ) renda sempre positivo o nullo il suo integrale relativo ad una qualsiasi curva;

$3^{\circ}$ ) renda minore di una quantita, arbitrariamente prefissabile, il suo integrale relativo alla curva $C_{0}$.

Rammentiamo, innanzi tutto, che, se inscriviamo nella curva $C_{o}$ una poligonale $i$ cui vertici abbiano ascisse crescenti, al tendere a zero del massimo lato di questa poligonale la fuuzione che definisce la poligonale stessa converge uniformemente alla $y_{0}(x)$, 
e la sua derivata converge in misura alla $y_{0}^{\prime}(x)$, vale a dire, la differenza tra la sua derivata e la $y_{0}^{\prime}(x)$ resta, in modulo, inferiore ad un $\varepsilon>0$ e comunque scelto, in un insieme di punti di $(a, b)$ la cui misura tende $\left.a b-a^{35}\right)$.

Preso allora ad arbitrio un numero positivo $\varepsilon$, inscriviamo nella curva $C_{0}$ una poligonale $\mathrm{i}$ cui vertici abbiano ascisse crescenti ed $\mathrm{i}$ cui lati siano sufficientemente piccoli, in modo che, smussati gli angoli con curve, in ciascuna delle quali la tangente vari sempre nello stesso senso, si abbia una curva rappresentata da una equazione.

$$
y=\pi_{\varepsilon}(x),
$$

con $\pi_{\varepsilon}(x)$ funzione continua insieme con le sue derivate prima e seconda, soddisfacente, in tutto $(a, b)$, alla disuguaglianza

$$
\left|y_{0}(x)-\pi_{\varepsilon}(x)\right|<\varepsilon,
$$

e in tutto $(a, b)$ ad eccezione al più di un insieme di punti $\mathbb{E}_{\varepsilon}$ di misura $m\left(\mathbb{E}_{\varepsilon}\right)<\varepsilon$, anche all'altra

$$
\left|y_{0}^{\prime}(x)-\pi_{\varepsilon}^{\prime}(x)\right|<\varepsilon .
$$

Indicando con $R$ un numero positivo di cui resti sempre inferiore il modulo della derivata $y_{0}^{\prime}(x)$, considerata là soltanto dove esiste, abbiamo anche, per la costruzione fatta,

e cio in tutto $(a, b)$.

$$
\left|\pi_{\varepsilon}^{\prime}(x)\right| \leq R,
$$

Poniamo, allora,

$$
E\left(x, y ; \pi_{\varepsilon}^{\prime}, y^{\prime}\right) \equiv f\left(x, y, y^{\prime}\right)-f\left(x, y, \pi_{\varepsilon}^{\prime}\right)-\left(y^{\prime}-\pi_{\varepsilon}^{\prime}\right) f_{y^{\prime}}\left(x, y, \pi_{\varepsilon}^{\prime}\right) .
$$

Essendo, per lo sviluppo del TaYlor,

$$
E\left(x, y ; \pi_{\varepsilon}^{\prime}, y^{\prime}\right)=\frac{1}{2}\left(y^{\prime}-\pi_{\varepsilon}^{\prime}\right)^{2} f_{y^{\prime} y^{\prime}}\left(x, y, \pi_{\varepsilon}^{\prime}+\theta\left(y^{\prime}-\pi_{\varepsilon}^{\prime}\right)\right),
$$

con $o<\theta<\mathrm{I}$, segue, dalla condizione posta nell'enunciato della nostra proposizione, che è sempre

$$
E\left(x, y ; \pi_{\varepsilon}^{\prime}, y^{\prime}\right) \supseteq \mathrm{o} .
$$

Detto $M$ il massimo modulo di $f_{y^{\prime}}\left(x, y_{0}(x), y^{\prime}\right)$ nel campo

$$
a \leq x \leq b, \quad\left|y^{\prime}\right| \leq R,
$$

abbiamo, sul complementare dell'insieme $\mathfrak{E}_{\mathrm{\varepsilon}}$ rispetto all'intervallo $(a, b)$, sul quale com-

35) Cfŕr. L. Tonelli, Successioni di curve e derivazioni per serie Nota II (Rendiconti della R. Accademia dei Lincei, vol. XXV, $1^{\circ}$ semestre I9I6, pp. 85-91). 
plementare è verificata la (32)

e quindi

$$
E\left(x, y_{0}(x) ; \pi_{\varepsilon}^{\prime}(x), y_{o}^{\prime}(x)\right)<2 \varepsilon M,
$$

$$
\begin{aligned}
\int_{a}^{b} E\left(x, y_{0}(x) ; \pi_{\varepsilon}^{\prime}(x), y_{0}^{\prime}(x)\right) d x & <2 \varepsilon M(b-a)+4 R M m\left(E_{\varepsilon}\right) \\
& <2 M((b-a)+2 R) \varepsilon .
\end{aligned}
$$

Sia ora $C$ un'altra qualsiasi curvà $\left(\mathrm{n}^{\circ} 40\right)$ avente per equazione

È per la (33)

$$
y=y(x) \text {. }
$$

$$
\int_{a}^{b} E\left(x, y(x) ; \pi_{\varepsilon}^{\prime}(x), y^{\prime}(x)\right) d x \supseteq 0
$$

ed anche per la (34)

$$
\begin{gathered}
\int_{a}^{b} E\left(x, y(x) ; \pi_{\varepsilon}^{\prime}(x), y^{\prime}(x)\right) d x- \\
-\int_{a}^{b} E\left(x, y_{0}(x) ; \pi_{\varepsilon}^{\prime}(x), y_{0}^{\prime}(x)\right) d x>-2 M(b-a+2 R) \varepsilon .
\end{gathered}
$$

Si ha dunque, dalla definizione stessa della funzione $E$,

$$
\begin{gathered}
\int_{a}^{b} f\left(x, y, y^{\prime}\right) d x-\int_{a}^{b} f\left(x, y_{0}, y_{0}^{\prime}\right) d x>-2 M(b-a+2 R) \varepsilon \\
\quad+\int_{a}^{b}\left\{f\left(x, y, \pi_{\varepsilon}^{\prime}\right)-\pi_{\varepsilon}^{\prime} f_{y^{\prime}}\left(x, y, \pi_{\varepsilon}^{\prime}\right)+y^{\prime} f_{y^{\prime}}\left(x, y, \pi_{\varepsilon}^{\prime}\right)\right\} d x- \\
-\int_{a}^{b}\left\{f\left(x, y_{0}, \pi_{\varepsilon}^{\prime}\right)-\pi_{\varepsilon}^{\prime} f_{y^{\prime}}\left(x, y_{0}, \pi_{\varepsilon}^{\prime}\right)+y_{o}^{\prime} f_{y^{\prime}}\left(x, y_{0}, \pi_{\varepsilon}^{\prime}\right)\right\} d x .
\end{gathered}
$$

Osserviamo quì che l'integrale

$$
\int_{a}^{b}\left\{\left[f\left(x, y, \pi_{\varepsilon}^{\prime}(x)\right)-\pi_{\varepsilon}^{\prime} f_{y^{\prime}}\left(x, y, \pi_{\varepsilon}^{\prime}(x)\right)\right]+y^{\prime} f_{y^{\prime}}\left(x, y, \pi_{\varepsilon}^{\prime}(x)\right)\right\} d x,
$$

essendo $\pi_{\varepsilon}^{\prime}$ e $\pi_{c}^{\prime \prime}$ funzioni continue, per costruzione, soddisfa alla condizione dell'enunciato del $n^{\circ} 43$ ed è pertanto una funzione continua della curva d'integrazione.

Potremo dunque determinare un numero $\rho>0$ in modo che, per ogni curva $C$, di equazione $y=y(x)$, appartenente all'intorno $(\rho)$ della $C_{o}$, la differenza degli integrali del secondo membro dell'ultima disuguaglianza scritta risulti, in valore assoluto, minore di $\varepsilon$.

E per tutte queste curve varrd la disuguaglianza

$$
\int_{a}^{b} f\left(x, y, y^{\prime}\right) d x-\int_{a}^{b} f\left(x, y_{0}, y_{o}^{\prime}\right) d x>-[2 M(b-a+2 R)+1] \varepsilon,
$$

il che dimostra precisamente la semicontinuità inferiore di $I_{C}$ sulla curva $C_{0}$. 
49. Si tratta ora di liberare la proposizione dimostrata al $\mathrm{n}^{\circ}$ precedente dalla condizione che la $y_{0}(x)$ abbia numeri derivati limitati. Per riuscire a ciò è necessario considerare dapprima una forma particolare della funzione $f\left(x, y, y^{\prime}\right)$.

Dimostriamo pertanto che:

Se in ogni parte limitata del campo $A$ la derivata parziale $f_{y^{\prime}}\left(x, y, y^{\prime}\right)$ resta limitata in modulo, e cio per tutti $i$ possibili valori di $y^{\prime}$, se, inoltre, è sempre, in tutto $A$ $e$ per tutti gli $\left.y^{\prime}, f_{y^{\prime} y^{\prime}} \gg 0^{36}\right)$, l'integrale $I_{C}$ è funzione semicontinua inferiormente della curva d'integrazione.

Considerata una curva $C_{0}\left(\mathrm{n}^{\circ} 40\right)$ di equazione $y=y_{0}(x)$, inscriviamo in essa una poligonale avente $i$ vertici ad ascisse crescenti e provenienti dalla divisione in $n$ parte uguali del segmento $(a, b)$. Sia $y=\pi_{n}(x)$ l'equazione di questa poligonale.

Dall'ipotesi fatta sulla derivata $f_{y^{\prime}}$, scende che, se $\rho$ è un numero positivo e consideriamo il campo $A^{\prime}$ costituito dai punti di $A$ di ascissa $x$ compresa fra $a$ e $b$ (estremi inclusi) e di ordinata compresa fra $y_{0}(x)-p$ e $y_{0}(x)+p$ (estremi inclusi), detto $M$ il limite superiore dei moduli di $f_{y^{\prime}}$ in $A^{\prime}$ e per tutti i possibili ${ }^{\prime} y^{\prime}$, abbiamo in $A^{\prime}$

$$
\begin{gathered}
f\left(x, y, y^{\prime}\right)=f(x, y, 0)+y^{\prime} f_{y^{\prime}}\left(x, y, \overline{y^{\prime}}\right), \\
\left|f\left(x, y, y^{\prime}\right)\right| \leqslant|f(x, y, o)|+M\left|y^{\prime}\right| .
\end{gathered}
$$

Abbiamo dunque che, per tutti gli $y^{\prime}$ in modulo superiori al massimo di $|f(x, y, o)|$ in $A^{\prime}$, è, in questo campo,

$$
\left|f\left(x, y, y^{\prime}\right)\right|<(M+\mathrm{I})\left|y^{\prime}\right| \text {. }
$$

Rileviamo, inoltre, che la curva $C_{0}$, per essere $y_{0}(x)$ funzione assolutamente continua, ha lunghezza finita; che la funzione $\pi_{n}(x)$, per $n \rightarrow \infty$, converge uniformemente, in tutto $(a, b)$, verso la $y_{0}(x)$, e che la lunghezza della poligonale corrispondente converge alla lunghezza della $C_{\mathrm{o}}$. Ci troviamo dunque nelle condizioni volute per l'applicazione dei risultati contenuti nei $\mathrm{n}^{\mathrm{i}}$ I, 2, 3, 4 della Nota gia citata [loc. cit. $\left.{ }^{35}\right)$ ] e possiamo asserire che, preso un numero positivo $\varepsilon$, è possibile determinare un $\bar{n}$ tale che, per ogni $n>\bar{n}$ :

$I^{\circ}$ ) la poligonale $y=\pi_{n}(x)$ appartenga all'intorno $(\rho)$ della $C_{0}$;

$2^{\circ}$ ) l'insieme $\dot{E}_{n}$ dei punti di $(a, b)$ nei quali non $\dot{\mathrm{e}}\left|y_{0}^{\prime}(x)-\pi_{n}^{\prime}(x)\right|<\varepsilon$ renda soddisfatte le disuguaglianze

$$
\int_{\mathbb{a}_{n}}\left|\pi_{n}^{\prime}(x)\right| d x<\frac{\varepsilon}{2}, \quad \int_{\mathbb{E}_{n}}\left|y_{0}^{\prime}(x)\right| d x<\frac{\varepsilon}{2} ;
$$

${ }^{36}$ ) Per questa seconda condizione, la prima è equivalente a quest'altra, che in ogni parte limitata del campo $A$ sia $|f|<L\left|y^{\prime}\right|$, per tutti gli $y^{\prime}$ in modulo superiori ad un numero fisso $L_{1}$, i numeri $L$ ed $L_{\mathrm{x}}$ potendo variare con la parte del campo considerata. 
$\left.3^{\circ}\right)$ sia

$$
\left|\int_{a}^{b} f\left(x, y_{0}, y_{0}^{\prime}\right) d x-\int_{0}^{b} f\left(x, y_{0}, \pi_{n}^{\prime}\right) d x\right|<\frac{\varepsilon}{2} .
$$

Cio fatto smussiamo i vertici della poligonale $y=\pi_{n}(x)$, in modo che ne risulti una curva di equazione $y=\bar{\pi}_{n}(x)$ la quale:

$\left.\mathrm{I}^{\circ}\right)$ ammetta per la $\bar{\pi}_{n}(x)$ le derivate prima e seconda continue;

$2^{\circ}$ ) giaccia tutta nell'intorno $(p)$ della $C_{0}$;

$3^{\circ}$ ) renda soddisfatte le disuguaglianze

$$
\int_{\vec{E}_{n}}\left|\overline{\pi_{n}^{\prime}}(x)\right| d x<\varepsilon, \quad \int_{\bar{\Xi}_{n}}\left|y_{0}^{\prime}(x)\right| d x<\varepsilon
$$

dove $\overline{\mathscr{E}}_{n}$ rappresenta l'insieme dei punti di $(a, b)$ nei quali non è $\left|y_{0}^{\prime}(x)-\bar{\pi}_{n}^{\prime}(x)\right|<\varepsilon$;

$\left.4^{\circ}\right)$ soddisfi alla disuguaglianza

$$
\left|\int_{a}^{b} f\left(x, y_{0}, y_{0}^{\prime}\right) d x-\int_{a}^{b} f\left(x, y_{0}, \bar{\pi}_{n}^{\prime}\right) d x\right|<\varepsilon .
$$

Conservando alla funzione $E\left(x, y ; \pi_{n}^{\top}, y^{\prime}\right)$ il significato del $\mathrm{n}^{\circ}$ precedente, abbiamo ancora che è sempre

$$
E\left(x, y ; \bar{\pi}_{n}^{\prime}, y^{\prime}\right) \supseteq 0
$$

per tutti gli $(x y)$ di $A$ e per tutti i possibili $y^{\prime}$; ed abbiamo anche, per quello che precede,

$$
\left|\int_{\bar{E}_{n}}\left(y_{0}^{\prime}-\overline{\pi_{n}^{\prime}}\right) f_{y^{\prime}}\left(x, y_{0}, \overline{\pi_{n}^{\prime}}\right) d x\right| \leq 2 M \varepsilon .
$$

E siccome, indicando con $C\left(\overline{\mathbb{E}}_{n}\right)$ il complementare di $\overline{\mathbb{E}}_{n}$ rispetto ad $(a, b)$, è

$$
\left|\int_{C\left(\overline{\mathbb{E}}_{n}\right)}\left(y_{\mathrm{o}}^{\prime}-\bar{\pi}_{n}^{\prime}\right) f_{y^{\prime}}\left(x, y_{\circ}, \bar{\pi}_{n}^{\prime}\right) d x\right|<\varepsilon M(b-a),
$$

segue, sommando,

$$
\left|\int_{a}^{b}\left(y_{o}^{\prime}-\bar{\pi}_{n}^{\prime}\right) f_{y^{\prime}}\left(x, y_{0}, \bar{\pi}_{n}^{\prime}\right) d x\right|<M(2+b-a) \varepsilon_{0}
$$

Da questa disuguaglianza e dalla (35) deduciamo

$$
\int_{a}^{b} E\left(x, y_{0} ; \pi_{n}^{\prime}, y_{0}^{\prime}\right) d x<\{I+M(2+b-a)\} \varepsilon .
$$

Dopo ciò non vi è che da proseguire il ragionamento come al $\mathrm{n}^{\mathbf{0}}$ precedente per giungere a stabilire la proposizione enunciata.

50. Sfruttando la proposizione dimostrata or ora, possiamo pervenire al teorema generale: 
L'integrale $I_{C}$ quasi regolare - con $f_{y^{\prime} y^{\prime}} \supseteq 0-\dot{e}$ funzione semicontinua inferiormente della curva d'integrazione.

Riprendiamo la funzione $E$ già definita al $\mathrm{n}^{\circ} 4^{8}$ e consideriamo

$$
E\left(x, y ; o, y^{\prime}\right) \equiv f\left(x, y, y^{\prime}\right)-\left\{f(x, y, o)+y^{\prime} f_{v^{\prime}}(x, y, o)\right\} .
$$

Per quello che si è detto al $\mathrm{n}^{0}$ citato, dalla condizione $f_{y^{\prime} y^{\prime}} \supseteq 0$ segue che è, per ogni punto $(x y)$ del campo $A$ e per tutti i possibili $y^{\prime}$,

Se dunque poniamo

$$
E\left(x, y ; 0, y^{\prime}\right) \gg 0 .
$$

abbiamo:

$$
\bar{f}\left(x, y, y^{\prime}\right) \equiv E\left(x, y ; o, y^{\prime}\right)
$$

$\left.\mathrm{I}^{0}\right)$ per tutte le terne $x, y, y^{\prime}$ dette, è $\bar{f} \supseteq 0$;

$\left.2^{\circ}\right)$ in tutto $A, \dot{\mathrm{e}} \bar{f}(x, y, 0)=0$;

$\left.3^{\circ}\right) \bar{f}$ è funzione continua di $\left(x, y, y^{\prime}\right)$ in tutti $\mathrm{i}$ punti indicati più sopra ed ammette le derivate parziali continue $\bar{f}_{y^{\prime}}, \bar{f}_{y^{\prime} y^{\prime}}, \bar{f}_{y^{\prime} x}$, e cio come la funzione $f$ da cui deriva;

$\left.4^{0}\right)$ in $A$ è sempre $\bar{f}_{y^{\prime} y^{\prime}}=f_{y^{\prime} y^{\prime}} \geqslant 0$;

$\left.5^{\circ}\right)$ per $y^{\prime}<\mathrm{o} \dot{e}$, in $A$, sempre $\bar{f}_{y^{\prime}} \leqslant \mathrm{o}$, e per $y^{\prime}>0$, sempre $\bar{f}_{y^{\prime}} \supseteq 0$ [come ririsulta da $\left.4^{\circ}\right)$ e da $f_{y^{\prime}}(x, y, 0)=0$;

$\left.6^{\circ}\right) \dot{\mathrm{e}}$

$$
\int_{C} \bar{f} d x=\int_{C} f d x-\int_{C}\left\{f(x, y, 0)+y^{\prime} f_{y^{\prime}}(x, y, o)\right\} d x ;
$$

e siccome l'ultimo integrale, soddisfacendo alle condizioni della proposizione del $\mathrm{n}^{\circ} 43$, è funzione continua della curva $C$, se noi dimostreremo la semicontinuita inferiore di $\int_{C} \bar{f} d x$ risulterà dimostrata anche quella di $\int_{C} f d x$.

Sia $C_{\text {o }}$ una curva $\left(\mathrm{n}^{\circ} 40\right)$ del campo $A$. Scelto ad arbitrio un numero positivo $\varepsilon$, possiamo determinare, per l'assoluta continuità della funzione $y_{0}(x)$ e per la esistenza dell'integrale

$$
\bar{I}_{C_{\mathrm{o}}}=\int_{C_{\mathrm{o}}} \bar{f} d x
$$

(conseguenza della supposta esistenza di $I_{C_{0}}$ ), un numero positivo $R$ tale che si abbia

$$
\int_{\mathscr{E}} \bar{f}\left(x, y_{0}, y_{0}^{\prime}\right) d x<\varepsilon
$$

dove $\mathbb{E}$ rappresenta l'insieme dei punti di $(a, b)$ nei quali o manca la derivata $y_{0}^{\prime}$ oppure questa derivata ha un valore assoluto $\supseteq R$.

Definiamo allora, per ogni punto $(x, y)$ di $A$ appartenente ad un intorno ( $\rho)$ della $C_{o}$, comunque scelto, una funzione $g\left(x, y, y^{\prime}\right)$ la quale sia: 
a) uguale a $\bar{f}$, per ogni $y^{\prime}$ tale che $\left|y^{\prime}\right| \leqslant R$;

b) minore o uguale a $\bar{f} \mathrm{e} \geqslant \mathrm{o}$, per $\left|y^{\prime}\right|>R$;

c) lineare rispetto ad $y^{\prime}$, per $\left|y^{\prime}\right| \geqslant 2 R$;

d) finita e continua, insieme con le sue derivate parziali $g_{y^{\prime}}, g_{y^{\prime} y^{\prime}}, g_{y^{\prime} x}$, in tutto un intorno $\left(\rho_{\mathrm{r}}\right)$ di $C_{0}$, con $\rho_{\mathrm{r}} \leqslant \rho$, e per tutti i possibili valori di $y^{\prime}$;

e) soddisfacente sempre, nell'intorno ora detto, alla disuguaglianza $g_{y^{\prime} y^{\prime}} \supseteq 0$.

Ciò è possibile in un'infinità di modi ${ }^{37}$ ).

Siccome per $c$ ) e $d$ ) possiamo determinare un numero positivo $M$ tale che sia sempre, in tutto l'intorno $\left(p_{1}\right)$ di $C_{0}$,

$$
\left|g_{y^{\prime}}\left(x, y, y^{\prime}\right)\right|<M,
$$

dalla proposizione del $\mathrm{n}^{\circ} 49$ segue che l'integrale $\int_{C} g\left(x, y, y^{\prime}\right) d x$, il quale per $\left.a\right)$ e $b$ ) esiste sulle curve sulla quale esiste $I_{C}$ e quindi $\bar{I}_{C}$, è sulla curva $C_{\mathrm{o}}$ una funzione semicontinua inferiormente. $\dot{E}$ dunque possibile determinare un numero positivo $p_{2}<p_{1}$ tale che, per ogni curva $C\left(n^{\circ} 4^{0}\right)$ appartenente all'intorno $\left(\rho_{2}\right)$ di $C_{0}$, si abbia

$$
\int_{C} g d x>\int_{C_{o}} g d x-\varepsilon
$$

Osserviamo che è, da una parte,

$$
\int_{C} g d x \leq \int_{C} \bar{f} d x
$$

e dall'altra, indicando con $C(\mathbb{E})$ il complementare, rispetto ad $(a, b)$, dell'insieme $\mathbb{E}$,

$$
\int_{C_{0}} g d x=\int_{C_{(\circledast)}} g\left(x, y_{0}, y_{0}^{\prime}\right) d x+\int_{\mathbb{E}} g\left(x, y_{0}, y_{0}^{\prime}\right) d x
$$

e per $a$ ) e $b$ ),

e ancora per (36)

$$
\int_{C_{0}} g d x \supseteq \int_{C_{(๕)}} \bar{f}\left(x, y_{0}, y_{0}^{\prime}\right) d x
$$

(39)

$$
\int_{C_{\mathrm{o}}} g d x \supseteq \int_{C_{\mathrm{o}}} \bar{f} d x-\varepsilon
$$

37) $\mathrm{Si}$ puó prendere ad esempio, per

$$
R \leqslant y^{\prime} \leqslant 2 R, \quad g=2 \bar{f}-\frac{y^{\prime}}{R} \bar{f}+\frac{2}{R} \int_{R}^{y^{\prime}} \bar{f} d y^{\prime}-\frac{y^{\prime}}{R} \vec{f}(R)+\bar{f}(R),
$$

e per $-2 R \leqslant y^{\prime} \leqslant-R$ la stessa funzione in cui si cambi $R$ su $-R_{j}$ per

$$
2 R \leq y^{\prime}, \quad g=\frac{y^{\prime}}{R}\{\overline{f(2 R)}-\bar{f}(R)\}-2 \bar{f}(2 R)+\bar{f}(R)+\frac{2}{R} \int_{R}^{2 R} \bar{f} d y^{\prime},
$$

c analogamente per $y^{\prime} \leqslant-2 R$. 
$\mathrm{Da}(37),(38)$ e (39) segue immediatamente

$$
\int_{C} \bar{f} d x>\int_{C_{0}} \bar{f} d x-2 \varepsilon
$$

la quale disuguaglianza dimostra la semicontinuità di $\bar{I}_{C}$ sulla curva $C_{\mathrm{o}}$ e quindi, per quanto abbiamo gid osservato, anche quella di $I_{C}{ }^{38}$ ).

\section{$\int 4$.}

\section{Condizioni sufficienti per la semicontinuità.}

c) La semicontinuità. su una data curva.

51. E evidente che se in tutto un'intorno di una curva $C_{o}\left(\mathrm{n}^{\circ} 4^{\circ}\right)$ risulta soddisfatta la condizione $f_{y^{\prime} y^{\prime}} \gg 0$, per tutti i possibili valori di $y^{\prime}$, l'integrale $I_{C}$ è funzione semicontinua inferiormente sulla curva $C_{0}$. Vogliamo ora occuparci di un caso notevole in cui non sempre la condizione ora menzionata è soddisfatta. E precisamente vogliamo dimostrare la proposizione che segue:

Sia $C_{\mathrm{o}}$ una curva $\left(\mathrm{n}^{\circ} 40\right)$ avente per equazione $y=y_{0}(x)$. Se:

a) per ogni punto $\bar{x}$ di $(a, b)$ in cui esiste finita la derivata $y_{0}^{\prime}(\bar{x})$, si pui determinare un numero positivo $\rho(\bar{x})$ tale che, per tutte le terne $\left(x, y, y^{\prime}\right)$ soddisfacenti alle disuguaglianze

$$
\begin{gathered}
|x-\bar{x}| \leqslant p(\bar{x}), \quad\left|y-y_{0}(\bar{x})\right| \leqslant p(\bar{x}), \\
\left|y^{\prime}-y_{0}^{\prime}(\bar{x})\right| \leqslant p(\bar{x}),
\end{gathered}
$$

e per le quali il punto $(x y)$ risulta appartenente al campo $A$, e per $\bar{y}^{\prime} \neq y^{\prime}$ sia (40) $E\left(x, y ; y^{\prime}, \bar{y}^{\prime}\right) \equiv f\left(x, y, \bar{y}^{\prime}\right)-f\left(x, y, y^{\prime}\right)-\left(\hat{y}^{\prime}-y^{\prime}\right) f_{y^{\prime}}\left(x, y, y^{\prime}\right)>0$;

b) per ogni punto $\overline{\bar{x}} d i(a, b)$ dove $y_{0}^{\prime}(x)$ i infinita o manca, esistono due numeri $\overline{\overline{y^{\prime}}}$ e $p(\overline{\bar{x}})$, dei quali il secondo positivo, in modo che soddisfatte le disuguaglianze

$$
\begin{gathered}
|x-\overline{\bar{x}}| \leqslant \rho(\overline{\bar{x}}), \quad\left|y-y_{0}(\overline{\bar{x}})\right| \leq \rho(\overline{\bar{x}}), \\
\left|y^{\prime}-\overline{y^{\prime}}\right| \leq \rho(\overline{\bar{x}}),
\end{gathered}
$$

ed appartenendo $(x y)$ al campo $A$ sia, per ogni $\overline{y^{\prime}} \neq y^{\prime}$, ancora verificata la $(40)$;

${ }^{\left.3^{8}\right)}$ Quando si abbia sempre $f_{y^{\prime} y^{\prime}} \geq m>0$, ed anche in casi più generali, la dimostrazione della semicontinuità di $I_{c}$ si puó ottenere più brevemente deducendola da un lemma estensione di quello del $\mathrm{n}^{\circ} 8$ [Cfr. loc. cit. $\left.\left.{ }^{4}\right)\right]$. 
allora l'integrale $I_{C}$ risulta funzione semicontinua inferiormente sulla curva $C_{\mathrm{o}}$.

La funzione $p(x)$ definita in questo enunciato la supporremo misurabile (nel senso del Lebesgue).

Consideriamo un punto $\bar{x}$ di $(a, b)$ ove esiste finita la derivata $y_{0}^{\prime}(\bar{x})$. La funzione $f\left(\bar{x}, y_{0}(\bar{x}), y^{\prime}\right)$ della sola $y^{\prime}$, per le condizioni poste, ha una derivata $f_{y^{\prime}}$ sempre crescente in tutto l'intervallo $\left(y_{0}^{\prime}(\bar{x})-p(\bar{x}), y_{0}^{\prime}(\bar{x})+p(\bar{x})\right)$. Ed infatti, se $y_{x}^{\prime}$ e $y_{2}^{\prime}$ sono due valori qualsiasi di questo intervallo, con $y_{1}^{\prime}<y_{2}^{\prime}$, è, per la (40),

$$
E\left(\bar{x}, y_{0}(\bar{x}) ; y_{1}^{\prime}, y_{2}^{\prime}\right)>0
$$

e quindi, per essere (Cfr. $\mathrm{n}^{\circ} 4^{8}$ )

$$
\begin{gathered}
E\left(\bar{x}, y_{\mathrm{o}}(\bar{x}) ; y_{1}^{\prime}, y_{2}^{\prime}\right)=\frac{1}{2}\left(y_{2}^{\prime}-y_{\mathrm{s}}^{\prime}\right)^{2} f_{y^{\prime} y^{\prime}}\left(\bar{x}, y_{\mathrm{o}}(\bar{x}), y_{\mathrm{s}}^{\prime}+\theta\left(y_{2}^{\prime}-y_{\mathrm{r}}^{\prime}\right)\right), \\
f_{y^{\prime} y^{\prime}}\left(\bar{x}, y_{\mathrm{o}}(\bar{x}), y_{\mathrm{s}}^{\prime}+\theta\left(y_{2}^{\prime}-y_{\mathrm{r}}^{\prime}\right)\right)>0
\end{gathered}
$$

e facendo tendere $y_{2}^{\prime}$ a $y_{1}^{\prime}$

$$
f_{y^{\prime} y^{\prime}}\left(\bar{x}, y_{0}(\bar{x}), y_{s}^{\prime}\right) \gg 0 \text {. }
$$

E dunque, nell'intervallo detto, sempre $f_{y^{\prime} y^{\prime}}\left(\bar{x}, y_{0}(\bar{x}), y^{\prime}\right) \gg 0$ senza che [per la (4I)] l'uguaglianza possa esser sempre verificata in tutto un segmento parziale di esso. La $f_{y^{\prime}}$ è perciò, in quell'intervallo, sempre crescente ed è (42) $f_{y^{\prime}}\left(\bar{x}, y_{0}(\bar{x}), y_{0}^{\prime}(\bar{x})-p(\bar{x})\right)<f_{y^{\prime}}\left(\bar{x}, y_{0}(\bar{x}), y_{0}^{\prime}(\bar{x})\right)<f_{y^{\prime}}\left(\bar{x}, y_{0}(\bar{x}), y_{0}^{\prime}(\bar{x})+p(\bar{x})\right)$. Essendo poi

$$
\begin{aligned}
& E\left(\bar{x}, y_{0}(\bar{x}), y_{0}^{\prime}(\bar{x})-p(\bar{x}), \widehat{y^{\prime}}\right)>0, \\
& E\left(\bar{x}, y_{0}(\bar{x}), y_{0}^{\prime}(\bar{x})+p(\bar{x}), \overline{y^{\prime}}\right)>0,
\end{aligned}
$$

se consideriamo in un piano la curva rappresentativa della funzione $f\left(\bar{x}, y_{0}(\bar{x}), \overline{y^{\prime}}\right)$, dove assumiamo come variabile indipendente la $\bar{y}^{\prime}$, la tangente $r_{1}$ a questa curva nel punto corrispondente a $\bar{y}^{\prime}=y_{0}^{\prime}(\bar{x})-\rho(\bar{x})$ giace tutta al disotto della curva medesima, e lo stesso accade per la tangente $r_{2}$ nel punto corrispondente a $\overline{y^{\prime}}=y_{\mathrm{c}}^{\prime}(\bar{x})+p(\bar{x})$.

$\mathrm{Ne}$ viene dunque, per le (42), che, essendo il primo e l'ultimo termine di tali disuguaglianze $i$ coefficienti angolari della retta $r_{1}$ e $r_{2}$, per $\bar{y}^{\prime} \leq y_{0}^{\prime}(\bar{x})-p(\bar{x})$ l'ordinata di $r_{1}$ è sempre maggiore di quella di $r_{2}$, e per $\bar{y}^{\prime} \geqslant y_{0}^{\prime}(\bar{x})+\rho(\bar{x})$ è invece l'ordinata di $r_{2}$ sempre maggiore di quella di $r_{1}$. Pertanto la retta $r$ che ha per ordinata generica la semisomma delle ordinate di $r_{1}$ e $r_{2}$, e cioè

$$
\begin{aligned}
\frac{1}{2}\left\{f\left(\bar{x}, y_{0}(\bar{x}), y_{0}^{\prime}(\bar{x})+p(\bar{x})\right)+f\left(\bar{x}, y_{0}(\bar{x}), y_{0}^{\prime}(\bar{x})-p(\bar{x})\right)\right\} \\
+\frac{1}{2}\left\{\left(\bar{y}^{\prime}-y_{0}(\bar{x})-p(\bar{x})\right) f_{y^{\prime}}\left(\bar{x}, y_{0}(\bar{x}), y_{0}^{\prime}(\bar{x})+p(\bar{x})\right)\right. \\
\left.+\left(\overline{y^{\prime}}-y_{0}^{\prime}(\bar{x})+p(\bar{x})\right) f_{y^{\prime}}\left(\bar{x}, y_{0}(\bar{x}), y_{0}^{\prime}(\bar{x})-p(\bar{x})\right)\right\},
\end{aligned}
$$


resta tutta al disotto della curva rappresentativa della funzione $f\left(\bar{x}, y_{\circ}(\bar{x}), \bar{y}^{\prime}\right)$, e la differenza fra le ordinate di questa curva e della retta $r$ si mantiene maggiore di un numero positivo.

Sia $p_{1}(\bar{x})$ un numero positivo $\leqslant p(\bar{x})$ e tale che, soddisfatte le disuguaglianze

$$
|x-\bar{x}| \leqslant p_{1}(\bar{x}), \quad\left|y-y_{0}(x)\right| \leqslant p_{1}(\bar{x}),
$$

la $f\left(x, y, \bar{y}^{\prime}\right)$ risulti sempre maggiore di quell'ordinata della retta $r$ detta che corrisponde allo stesso valore di $\overline{y^{\prime}}$, se $(x y)$ appartiene ad $A$. Perchè ciò sia basterà prendere. $\mathrm{p}_{\mathrm{t}}(\bar{x})$ in modo che, soddisfatte le disuguaglianze precedenti, la funzione $f\left(x, y, \bar{y}^{\prime}\right)$ sia maggiore della corrispondente ordinata della retta $r$, per ogni $\bar{y}^{\prime}$ dellintervallo $\left\{y_{0}^{\prime}(\bar{x})-p(\bar{x}), y_{0}^{\prime}(\bar{x})+p(\bar{x})\right\}$, e le tangenti alla curva che rappresenta $f\left(x, y, \bar{y}^{\prime}\right)$ come funzione della $\bar{y}^{\prime}$, nei punti corrispondenti a $\bar{y}^{\prime}=y_{0}^{\prime}(\bar{x})-p(\bar{x})$ e $\bar{y}^{\prime}=y_{0}^{\prime}(\bar{x})+p(\bar{x})$, abbiano ordinate maggiori della retta $r$, la prima per ogni $\bar{y}^{\prime} \leqslant y_{0}^{\prime}(\bar{x})-\rho(\bar{x})$, la seconda per ogni $\bar{y}^{\prime}>y_{0}^{\prime}(\bar{x})+\rho(\bar{x})$. Si convenga, per fissare le idee, di scegliere $\rho_{1}(\bar{x})$ uguale alla metà del limite superiore dei valori per esso possibili.

Quanto si è fatto per i punti $\bar{x}$, si ripeta per quelli $\overline{\bar{x}}$ nei quali la derivata $y_{0}^{\prime}(x)$ o manca o non è finita, sostituendo nel ragionamento ad $y_{0}^{\prime}(\bar{x})$ il valore $\overline{y^{\prime}}$.

Ne viene cosi che, per ogni punto $x_{\circ}$ di $(a, b)$ (sia esso un $\bar{x} \circ$ un $\overline{\bar{x}}$ ) risultano determinate una funzione $p_{\mathrm{I}}\left(x_{\mathrm{o}}\right)>\mathrm{o}$ e $<_{\mathrm{p}}\left(x_{\mathrm{o}}\right)$, e una retta $r_{\mathrm{o}}$, in modo che, per ogni ogni punto $(x, y)$ di $A$ soddisfacente alle disuguaglianze

$$
\left|x-x_{0}\right| \leqslant p_{1}\left(x_{0}\right), \quad\left|y-y_{0}\left(x_{0}\right)\right| \leqslant p_{r}\left(x_{0}\right),
$$

la $f\left(x, y, \bar{y}^{\prime}\right)$ sia, per tutti $\mathrm{i}$ valori di $\bar{y}^{\prime}$, maggiore della corrispondente ordinata della retta $r_{0}$.

Ad ogni punto $x_{0}$ si faccia anche corrispondere un numero $\rho_{2}\left(x_{0}\right)$, uguale al minore fra $\mathrm{i}$ due $\frac{s}{2} \rho_{\mathrm{r}}\left(x_{0}\right)$ e $\sigma\left(x_{0}\right)$, dove $\sigma\left(x_{0}\right)$ è positivo e tale che nell'intervallo $\left(x_{0}-\sigma\left(x_{0}\right), x_{0}+\sigma\left(x_{0}\right)\right)$ l'oscillazione della funzione $y_{0}(x)$ sia $\leqslant \frac{1}{2} p_{1}\left(x_{0}\right)$.

In base ad un noto teorema di BoREL ${ }^{39}$ ) potremo allora scegliere un numero finito di punti di $(a, b): x_{1}, x_{2}, \ldots, x_{n}$, in modo che gli intervalli

$$
\left\{x_{s}-p_{2}\left(x_{s}\right), x_{s}+p_{2}\left(x_{s}\right)\right\}, \quad(s=1,2, \ldots, n),
$$

ricoprano tutto l'intervallo $(a, b)$; e dopo aver soppresso le parti di ciascuno di essi

39) loc. cit. ${ }^{18}$ ). 
eventualmente ricoperte da altri intervalli dello stesso gruppo corrispondenti ad indici minori, risulteranno degli intervalli in numero finito

$$
\delta^{(\mathrm{r})}, \quad \delta^{(2)}, \ldots, \delta^{(m)}
$$

non sovrapponentisi, ricoprenti interamente il segmento $(a, b)$ e tali che ad essi corrispondano le rette

ed $\mathrm{i}$ numeri positivi

$$
r^{(\mathrm{I})}, \quad r^{(2)}, \ldots, r^{(m)}
$$

$$
p^{(1)}, \quad p^{(2)}, \ldots, p^{(m)},
$$

cosi che, per ogni $x$ di $\delta^{(s)}(s=\mathrm{I}, 2, \ldots, m)$, per ogni $y$ soddisfacente alla $\left|y-y_{0}(x)\right| \leq p^{(s)}$ e per tutti i valori finiti di $y^{\prime}$, la funzione $f\left(x, y, y^{\prime}\right)$ resti sempre maggiore dell'ordinata

$$
p^{(s)}+y^{\prime} q^{(s)}
$$

della retta $r^{(s)}, p^{(s)}$ e $q^{(s)}$ essendo delle costanti, indipendenti da $x, y, y^{\prime}$.

$E$ ben evidente che, per dimostrare la semicontinuità dell'integrale $I_{C}$ basta dimostrare quella dell'integrale corrispondente agli archi di curva che si proiettano sul segmento $\delta^{(s)}$; e siccome, per la proposizione del $n^{\circ} 43$, l'integrale della funzione $p^{(s)}+y^{\prime} q^{(s)}$ varia con continuita al variare della curva d'integrazione, basta anche provare la semicontinuità dell'integrale della funzione

$$
f^{(s)} \equiv f-\left(p^{(s)}+y^{\prime} q^{(s)}\right)
$$

relativamente all'intervallo $\delta^{(s)} 4^{\circ}$ ).

Se indichiamo con $E^{(s)}$ la funzione $E$ corrispondente alla $f^{(s)}$, abbiamo

$$
E^{(s)} \equiv E \text {. }
$$

40) Qualora la $y_{0}^{\prime}(x)$ esista ovunque e sia continua, la retta $r^{(s)}$ si puo scegliere in modo che in tutto $\delta^{(s)}$ si abbia sempre, prefissato con $\varepsilon<0$,

e quindi

$$
f\left(x, y_{0}(x), \quad y_{0}^{\prime}(x)\right)-\left(p^{(s)}+y_{0}^{\prime}(x) q^{(s)}\right)<\varepsilon
$$

$$
\int_{\delta^{(s)}} f\left(x, y_{0}(x), \quad y_{0}^{\prime}(x)\right) d x-\varepsilon<\int_{\delta^{(s)}}\left(p^{(s)}+y_{0}^{\prime}(x) q^{(s)}\right) d x .
$$

E siccome è, per ogni altra curva dell'intorno $\left(\rho^{(s)}\right)$ della $C_{o}$,

$$
\int_{\delta^{(s)}}\left(p^{(s)}+y^{\prime} q^{(s)}\right) d x<\int_{\delta(s)} f\left(x, y, y^{\prime}\right) d x
$$

e l'integrale della $p^{(s)}+y^{\prime} q^{(s)}$ è funzione continua della curva d'integrazione, ne viene senz'altro la continuità dell'integrale della $f$ sull'arco di $C_{0}$ che corrisponde a $\delta^{(s)}$. 
Ciò porta a concludere che, per dimostrare la nostra proposizione, si puó supporre la $f$ sempre positiva.

52. Ammessa questa ipotesi e preso ad arbitrio un numero positivo $\sigma$, scegliamone un altro, pure positivo, $R$ in modo che si abbia

$$
\int_{C(ङ)} f\left(x, y_{0}, y_{0}^{\prime}\right) d x<\sigma
$$

dove $C(\&)$ rappresenta il complementare dell'insieme $\&$ dei punti di $(a, b)$ nei quali la derivata $y_{0}^{\prime}(x)$ esiste ed è in valore assoluto $\leqslant R$. Questa scelta è possibile per l'assoluta continuita della funzione $y_{\mathrm{o}}(x)$ e dell'integrale

$$
\int_{a}^{x} f\left(x, y_{0}, y_{0}^{\prime}\right) d x
$$

Riprendendo il ragionamento fatto al $\mathrm{n}^{\circ}$ precedente si vede che ad ogni $\bar{x}$ di $\mathbb{s i}$ puó far corrispondere una funzione $\bar{p}+y^{\prime} \bar{q}$ - l'ordinata della retta $r-$ la quale sia sempre minore di $f\left(\bar{x}, y_{0}(\bar{x}), y^{\prime}\right)$, per ogni $y^{\prime}$. Si vede di più che, sostituendo al numero $p(\bar{x})$ un $p_{\mathrm{I}}(\bar{x})$ minore e convenientemente piccolo, si puó far in modo che, per ogni $y^{\prime}$ dell'intervallo $\left\{y_{0}^{\prime}(\bar{x})-p_{1}(x), y_{0}^{\prime}(\bar{x})+p_{1}(\bar{x})\right\}$, sia

$$
\circ<f\left(\bar{x}, y_{0}(\bar{x}), y^{\prime}\right)-\left(\bar{p}+y^{\prime} \bar{q}\right)<\sigma
$$

e il coefficiente angolare della retta $r$, ossia il numero $\bar{q}$, è poi sempre compreso fra $\mathrm{i}$ coefficienti angolari delle rette $r_{\mathrm{I}}$ e $r_{2}, \mathrm{i}$ quali sono dati $f_{y^{\prime}}\left(\bar{x}, y_{0}(\bar{x}), y_{0}^{\prime}(\bar{x})-p_{1}(\bar{x})\right)$ e $f_{y^{\prime}}\left(\bar{x}, y_{0}(\bar{x}), y_{0}^{\prime}(\bar{x})+p_{1}(\bar{x})\right)$. Prenderemo $p_{1}(\bar{x})$ minore anche di $R$.

Sia, allora, $\rho_{2}(\bar{x})$ un numero positivo minore di $\rho_{1}(\bar{x})$ e tale che; soddisfatte le disuguaglianze

$$
\begin{gathered}
|x-\bar{x}| \leqslant p_{2}(\bar{x}), \quad\left|y-y_{0}(\bar{x})\right| \leqslant p_{2}(\bar{x}), \\
y^{\prime}-y_{0}^{\prime}(\bar{x}) \mid \leqslant p_{2}(\bar{x}),
\end{gathered}
$$

( $x y$ ) essendo un punto di $A$ appartenente all'intorno $(p)$ della $C_{o}$, si abbia

$$
0<f\left(x, y, y^{\prime}\right) \dot{-}\left(\bar{p}+y^{\prime} \bar{q}\right)<\sigma
$$

$P_{2}(\bar{x})$ sia poi anche tale che per gli stessi punti $(x y)$ e per ogni $y^{\prime}$ si abbia

$$
f\left(x, y, y^{\prime}\right)-\left(\bar{p}+y^{\prime} \bar{q}\right)>0 .
$$

Per fissare le idee, scegliamo fra gli infiniti possibili $\rho_{\mathrm{I}}(\bar{x})$ la metà del loro limite superiore; e altrettanto facciamo per $p_{2}(\bar{x})$. E importante rilevare che, per essere 
$\left|y_{0}^{\prime}(\bar{x})\right| \leq R$ e per la condizione imposta a $p_{1}(\bar{x})$ di essere $<R$, è sempre

$$
|\bar{q}|<M^{\prime}
$$

dove con $M^{\prime}$ indichiamo il massimo modulo di $f_{y^{\prime}}$ nei punti dell'intorno $(\rho)$ della $C_{0}$ e per $\left|y^{\prime}\right| \leq 2 R$. In tutti $\mathrm{i}$ punti $\left(x, y, y^{\prime}\right)$ del campo ora indicato, la funzione $f\left(x, y, y^{\prime}\right)$ ha un massimo modulo; si ricava dunque dalla (45) che esiste anche un numero positivo $M^{\prime \prime}$ per il quale è sempre

$$
\bar{p} \mid<M^{\prime \prime} .
$$

Indicando con $M$ il maggiore dei due numeri $M^{\prime}, M^{\prime \prime}$, abbiamo sempre,

$$
|\bar{p}|<M, \quad|\bar{q}|<M .
$$

Indichiamo ora con $E_{n}$ l'insieme dei punti di $\mathbb{E}$ in cui è $\rho_{2}(\bar{x})>\frac{\mathrm{I}}{n}$.

Siccome è sempre $\rho_{2}(x)>0$ su $\mathfrak{E}$, la misura di $\dot{E}_{n}$ tende, per $n \rightarrow \infty$, a quella di $\&$. Sia $\bar{n}$ un valore di $n$ tale che

$$
\int_{C\left(\sigma_{\tilde{n}}\right)} f\left(x, y_{0}, y_{0}^{\prime}\right) d x<\sigma
$$

questo $\bar{n}$ esiste certamente perchè vale la $(43)$, dove $C(\mathbb{E})$ è contenuto in $C(\mathbb{E})$ ed ha una misura che è il limite, per $n \rightarrow \infty$, di quella di quest'ultimo insieme. In ogni punto di $F_{\bar{n}}$ è per definizione, $\rho_{2}(x)>\frac{\mathrm{I}}{\bar{n}}$.

Dopo ciò, indichiamo con $\rho_{3}$ un numero positivo minore di $\frac{\mathrm{I}}{4 \vec{n}}$, e con procedimento analogo a quello seguito al $\mathrm{n}^{\mathrm{o}}$ 49. per la determinazione della funzione $\bar{\pi}_{n}(x)$, definiamo una funzione $P(x)$, in tutto $(a, b)$, la quale:

$\left.I^{\circ}\right)$ sia continua insieme con la sua derivata prima;

$\left.2^{\circ}\right)$ soddisfi a tutto $(a, b)$ alla disuguaglianza $\left|y_{0}(x)-P(x)\right|<p_{3}$;

$\left.3^{0}\right)$ soddisfi alla disuguaglianza $\left|y_{0}^{\prime}(x)-P^{\prime}(x)\right|<\rho_{;}$in un componente chiuso $E_{\bar{n}, \mathrm{i}}$ di $E_{\bar{n}}$ il cui complementare verifichi ancora la $(48)$, vale a dire la

$$
\int_{C\left(\Xi_{\bar{n},}\right)} f\left(x, y_{0}, y_{0}^{\prime}\right) d x<\sigma .
$$

Per la continuità della $P(x)$ e della $P^{\prime}(x)$, è possibile di determinare un numero positivo $\delta$, minore di $\rho_{;}$, tale da rendere soddisfatte le disuguaglianze

$$
\left|P(x)-P\left(x^{\prime}\right)\right|<p_{3}, \quad\left|P^{\prime}(x)-P^{\prime}\left(x^{\prime}\right)\right|<p_{3},
$$


quando sia $\left|x-x^{\prime}\right| \leq \delta$; e in virtù del già citato teorema di Borel, si puó, fra tutti gli intervalli di ampiezza $\delta$ avente per centro un punto dell'insieme chiuso $\dot{C}_{\bar{n}_{\mathrm{I}}}$; sceglierne un numero finito cosi da ricoprire con esso tutto l'insieme detto.

$\mathrm{Si}$ ordinino, con un qualsiasi criterio, questi intervalli e da ciascuno di essi si tolgano quelle parti che risultassero sovrapposte ad altri intervalli di indice minore; si sopprima poi dai nuovi intervalli risultanti un certo numero di quelle loro parti che eventualmente non contenessero alcun punto $\mathrm{di} \varepsilon_{\bar{n}, \mathrm{I}}$, in modo che la somma delle lunghezze degli intervalli restanti $\omega_{1}, \omega_{2}, \ldots, \omega_{m}$, sia tanto prossima alla misura di $\mathbb{E}_{\bar{n}_{1},}$ che, detto $\mathscr{C}^{\prime}$ l'insieme dei punti di tutti questi $\omega$. che non appartengono a $\mathbb{E}_{\bar{n}, \mathrm{r}}$, si abbia

$$
m\left(\mathbb{F}^{\prime}\right)<\frac{\sigma}{M}, \quad \int_{\mathbb{E}^{\prime}}\left|y_{\mathrm{o}}^{\prime}\right| d x<\frac{\sigma}{M}, \quad \int_{\mathbb{E} \prime^{\prime}} f\left(x, y_{\mathrm{o}}, y_{\mathrm{o}}^{\prime}\right) d x<\sigma .
$$

Per la loro costruzione gli intervalli $\omega$ risultano non sovrapponentisi, ricoprenti interamente $\dot{E}_{\bar{n}, \mathrm{i}}$ e tutti di ampiezza minore di $\delta$.

Consideriamo uno qualunque di questi $\omega$, ad esempio $\omega_{s}$, e sia $x_{s}$ un punto di $F_{n, \mathrm{I}}$ appartenente ad esso (per esempio quello più a sinistra). Questo $x_{s}$ appartiene necessariamente anche a $\mathbb{F}_{\bar{n}}$ e a $\mathbb{E}$ : sia pertanto $p_{s}+y^{\prime} q_{s}$ la funzione $\bar{p}+y^{\prime} \bar{q}$ della disuguaglianza (44), che gli corrisponde. Detto $x$ un qualsiasi punto di $\omega_{s}$ appartenente a $\dot{E}_{\bar{n}, 1}$ è, per le proprietd $2^{\circ}$ ) e $3^{\circ}$ ) cui soddisfa la $P(x)$ e per le (49),

$$
\begin{array}{r}
\left|x-x_{s}\right|<\delta<p_{3}<\frac{\mathrm{I}}{4 \bar{n}}<p_{2}\left(x_{s}\right), \\
\left|y_{0}(x)-y_{0}\left(x_{s}\right)\right|<3 p_{3}<\frac{3}{4 \bar{n}}<p_{2}\left(x_{s}\right), \\
\left|y_{0}^{\prime}(x)-y_{0}^{\prime}\left(x_{s}\right)\right|<3 p_{3}<\frac{3}{4 \bar{n}}<p_{2}\left(x_{s}\right) .
\end{array}
$$

Dunque, per la $x$ detta, si ha (45)

$$
0<f\left(x, y_{\circ}(x), y_{0}^{\prime}(x)\right)-\left(p_{s}+y_{o}^{\prime} q_{s}\right)<\sigma .
$$

Se poi $x$ è un altro qualsiasi punto di $\omega_{s}$ ed è $\left|y-y_{0}(x)\right|<p_{3}$, avendosi

$$
\begin{gathered}
y-y_{0}\left(x_{s}\right)=\left(y-y_{0}(x)\right)+\left(y_{0}(x)-P(x)\right)+\left(P(x)-P\left(x_{s}\right)\right)+\left(P\left(x_{s}\right)-y_{0}\left(x_{s}\right)\right) \\
\left|y-y_{0}\left(x_{s}\right)\right|<4 p_{3}<\frac{\mathrm{I}}{\bar{n}}<p_{2}\left(x_{s}\right),
\end{gathered}
$$

'si ha, per la (46), e qualunque sia la $y^{\prime}$,

$$
f\left(x, y, y^{\prime}\right)-\left(p_{s}+y^{\prime} q_{s}\right)>0 .
$$

Rend. Circ. Matem. Palermo, t. XLIV (1920). - Stampato il 20 settembre 1920. 
Indichiamo con $\mathbb{F}^{(s)}$ la parte di $\mathbb{E}_{\bar{n}, \mathrm{I}}$ contenuta in $\omega_{s}$, con $\mathbb{F}_{\mathrm{r}}^{(s)}$, il complementare di $\mathbb{E}^{\langle(s)}$ rispetto a $\omega_{s}$. Abbiamo, per la $(5 \mathrm{I})$,

$$
0<\int_{\mathbb{E}^{(s)}} f\left(x, y_{0}(x), y_{0}^{\prime}(x)\right) d x-\int_{\mathbb{G}^{(s)}}\left(p_{s}+y_{0}^{\prime} q_{s}\right) d x<\sigma \cdot m\left(\xi^{(s)}\right),
$$

e per le $(47)$ e (52)

$$
\begin{gathered}
0<\int_{\omega_{s}} f\left(x, y_{0}(x), y_{0}^{\prime}(x)\right) d x-\int_{\omega_{s}}\left(p_{0}+y_{0}^{\prime} q_{s}\right) d x \\
<\sigma \cdot m\left(\mathscr{E}^{(s)}\right)+\int_{\mathscr{E}_{1}^{(s)}} f\left(x, y_{0}(x), y_{0}^{\prime}(x)\right) d x+M\left\{m\left(\mathbb{E}_{1}^{(s)}\right)+\int_{\mathscr{E}_{1}^{(s)}}\left|y_{0}^{\prime}\right| d x\right\},
\end{gathered}
$$

e sommando rispetto ad $s$ e tenendo conto delle (5o),

$$
\begin{aligned}
0<\sum_{s} \int_{\omega_{s}} f\left(x, y_{0}(x), y_{0}^{\prime}(x)\right) d x-\sum_{s} \int_{\omega_{s}}\left(p_{s}+y_{o}^{\prime} q_{s}\right) d x & <\sigma(b-a)+\sigma+2 \sigma \\
& =\sigma(b-a+3) .
\end{aligned}
$$

E poichè le parti di $(a, b)$ esterne agli intervalli $\omega_{s}$ sono comprese in $C\left(\mathbb{E}_{\bar{n}, \mathrm{I}}\right)$, dalla condizione $3^{\circ}$ ) cui soddisfa la $P(x)$, scende, per la disuguaglianza ora scritta,

(53) $0<\int_{a}^{b} f\left(x, y_{0}(x), y_{0}^{\prime}(x)\right) d x-\sum_{s} \int_{\omega_{s}}\left(p_{s}+y_{0}^{\prime} q_{s}\right) d x<\sigma(b-a+4)$.

Osserviamo ora che, essendo $p_{s}$ e $q_{s}$ delle costanti, l'integrale

$$
\int_{\omega_{s}}\left(p_{s}+y^{\prime} q_{s}\right) d x
$$

è $\left(\mathrm{n}^{\circ} 43\right)$ funzione continua della curva d'integrazione. Possiamo dunque determinare un intorno $(\bar{\rho})$ di $C_{0}$, con $\bar{\rho}<p_{3}$, in guisa che, per ogni curva $C$, di equazione $y=y(x)$, ad esso appartenente, sia per tutti gli indici $s$, da 1 ad $\dot{m}$,

$$
\left|\int_{\omega_{s}}\left(p_{s}+y_{o}^{\prime} q_{s}\right) d x-\int_{\omega_{s}}\left(p_{s}+y^{\prime} q_{s}\right) d x\right|<\frac{\sigma}{m}
$$

e perciò

$$
\left|\sum_{s} \int_{\omega_{s}}\left(p_{s}+y_{0}^{\prime} q_{s}\right) d x-\sum_{s} \int_{\omega_{s}}\left(p_{s}+y^{\prime} q_{s}\right) d x\right|<\sigma .
$$

Ma la (52) dd

$$
\sum_{s} \cdot \int_{\omega_{s}} f\left(x, y, y^{\prime}\right) d x-\sum_{s} \int_{\omega_{s}}\left(p_{s}+y^{\prime} q_{s}\right) d x>0,
$$


onde

$$
\sum_{s} \int_{\omega_{s}} f\left(x, y, y^{\prime}\right) d x>\sum_{s} \int_{\omega_{s}}\left(p_{s}+y_{0}^{\prime} q_{s}\right) d x-\sigma
$$

ed anche, per essere sempre $f \gg 0$,

$$
\int_{a}^{b} f\left(x, y, y^{\prime}\right) d x>\sum_{s} \int_{\omega_{s}}\left(p_{s}+y_{o}^{\prime} q_{s}\right) d x-\sigma .
$$

Da questa e dalla (s3) si ha

$$
\int_{a}^{b} f\left(x, y, y^{\prime}\right) d x>\int_{a}^{b} f\left(x, y_{0}, y_{0}^{\prime}\right) d x-\sigma(b-a+\varsigma)
$$

la quale risulta soddisfatta per ogni curva $C$ appartenente all'intorno $(\bar{\rho})$ della $C_{0}$.

La proposizione enunciata al $^{\circ}{ }^{\circ}$ I è dunque completamente provata.

53. Osservazione I. - Nell'enunciato della proposizione del $n^{0}$ s I si puó sostituire alla disuguaglianza $E\left(x, y ; y^{\prime}, \bar{y}^{\prime}\right)>0$ l'altra

$$
E\left(x, y ; y^{\prime}, \bar{y}^{\prime}\right) \supseteq 0
$$

e ciò tanto nella condizione $a$ ) che in quella $b$ ), purchè si abbia, per ogni $\hat{y}^{\prime} \neq y_{0}^{\prime}(x)$,

$$
E\left(x, y_{0}(x) ; y_{0}^{\prime}(x), \widehat{y^{\prime}}\right)>0
$$

e per ogni $\bar{y}^{\prime} \neq \overline{\bar{y}}$

$$
E\left(\overline{\bar{x}}, y_{0}(\overline{\bar{x}}) ; \overline{\overline{y^{\prime}}}, \overline{y^{\prime}}\right)>0
$$

Le $(54),(55)$ sono certamente soddisfatte se è $f_{y^{\prime} y^{\prime}}\left(\bar{x}, y_{0}(\bar{x}), y_{0}^{\prime}(\bar{x})\right)>0$, $f_{y^{\prime} y^{\prime}}\left(\overline{\bar{x}}, y_{0}(\overline{\bar{x}}), \overline{\overline{y^{\prime}}}\right)>0$.

Osservazione II. - La proposizione del $\mathrm{n}^{\circ}$ s I continua a sussistere:

$\left.I^{\circ}\right)$ se, fra $\mathrm{i}$ punti di $(a, b)$ in cui esiste finita la $y_{0}^{\prime}(x)$, ve n'è un insieme di misura nulla che, invece di soddisfare alla condizione $a$ ), soddisfano alla $b$ );

$\left.2^{\circ}\right)$ oppure se, essendo la funzione $f\left(x, y, y^{\prime}\right)$ sempre maggiore di un numero fisso $N$, è verificata soltanto la condizione $a)$, ed essa lo è in tutti i punti in cui esiste finita la $y_{0}^{\prime}(x)$, ad eccezione al più di un insieme di misura nulla.

Nell'uno e nell'altro di questi casi le condizioni di cui si tratta possono essere verificati nella forma dell'Osservazione $\mathrm{I}$.

54. Analogamente a quanto è stato notato al $\mathrm{n}^{\circ} 28$, per il caso delle curve in forma parametrica, si puó rilevare anche qui che, in forza dell'Osservazione II, ora fatta la condizione

$$
f_{y^{\prime} y^{\prime}}\left(x, y_{0}(x), y_{0}^{\prime}(x)\right) \supseteq 0
$$


puó non essere verificata su un insieme di punti di misura nulla, senza che per questo venga a mancare la semicontinuità inferiore di $I_{C}$ sulla curva $C_{0}$.

Lo stesso esempio addotto al $\mathbf{n}^{\mathbf{0}}$ citato serve anche per il caso attuale.

$$
\oint 5 \text {. }
$$

\section{Condizioni necessarie per la semicontinuità.}

55. Procedendo con lo stesso metodo seguito ai $n^{\mathrm{i}} 29$ e 32 , si provano le proposizioni che seguono:

a) Condizione necessaria affincbè su ogni curva $\left(n^{\circ} 40\right)$ del campo $A$ l'integrale $I_{C}$ sia funzione semicontinua inferiormente, $\dot{e}$ che si abbia

$$
f_{y^{\prime} y^{\prime}}\left(x, y, y^{\prime}\right) \supseteq 0
$$

per ogni $y^{\prime}$ fnito $e$ in tutti $i$ punti $(x y)$ interni al campo $A$ o limiti di punti interni.

b) Condizione necessaria affinchè su una data curva $C_{0}\left(n^{\circ} 40\right)$, completamente interna al campo $A$ e per la quale esista ovunque e sempre sia continua la derivata $y_{0}^{\prime}(x)$, lintegrale $I_{C}$ sia funzione semicontinua inferiormente, è che si abbia, in ogni punto di $C_{0}$.

$$
f_{y^{\prime} y^{\prime}}\left(x, y_{0}(x), y_{o}^{\prime}(x)\right) \gg 0
$$

Osservazione I. - I campi che comunemente si presentano sono tali che ogni loro punto o è interno o è punto limite di punti interni al campo stesso.

Per tali campi, la condizione trovata in a) come necessaria $\dot{e}$ anche sufficiente $\left(n^{\circ}\right.$ so) per la semicontinuità inferiore di $I_{C}$ su ogni curva appartenente al campo considerato.

Osservazione II. - La proposizione $b$ ) sussiste anche se la curva $C_{\mathrm{o}}$ ha un numero finito di punti non interni al campo $A$ o, più generalmente, se tali suoi punti si proiettano ortogonalmente sull'asse $x$ in un insieme di misura nulla. Essa vale pure se, fissato ad arbitrio un intorno $(\rho)$ della $C_{o}$, si puó sempre trovare una poligonale appartenente a quest'intorno, completamente interna ad $A$ e tale che sia incontrata in un sol punto al più da ogni parallela all'asse $y$, che i suoi lati abbiano coefficienti angolari inferiori in modulo ad un numero fisso, indipendente $d a p$, e che la sua lunghezza differisca da quella della curva $C_{o}$ per meno di $\rho$. La stessa proposizione ha poi piena validità, qualunque sia la curva $C_{\mathrm{o}}\left(\mathrm{n}^{\circ} 40\right)$ del campo $A$, se la frontiera di questo campo soddisfa alle condizione $b$ ) del $\mathrm{n}^{\circ} 40 \mathrm{e}$, oltre ad eventuali, segmenti rettilinei paralleli all'asse $y$, in numero finito, ha al più un numero, pure finito, di punti con tangente parallela al medesimo asse.

56. Si puó inoltre dimostrare, analogamente a quanto si è fatto al $n^{\circ} 33$, che: 
Condizione necessaria affincbè, su una data curva $C_{\mathrm{o}}\left(n^{\circ} 40\right)$ completamente interna al campo $A$, l'integrale $I_{C}$ sia funzione semicontinua inferiormente, $\dot{e}$ che risulti di misura nulla l'insieme dei valori di $x$ nei quali non è verificata la disuguaglianza.

$$
f_{y^{\prime} y^{\prime}}\left(x, y_{0}(x), y_{0}^{\prime}(x)\right) \supseteq 0 .
$$

Supponiamo che la misura dei punti dell'asse $x$ per i quali esiste finita la derivata $y_{0}^{\prime}(x)$ ed è

$$
f_{y^{\prime} y^{\prime}}\left(x, y_{0}(x), y_{0}^{\prime}(x)\right)<0 .
$$

non sia nulla. Indicatala con $2 \mu$ possiamo un insieme chiuso $E$ di questi punti tale che abbia misura $>\mu$ e che in esso sia sempre $f_{y^{\prime} y^{\prime}}\left(x, y_{0}(x), y_{0}^{\prime}(x)\right)<-\frac{3}{2} n$ il modulo di $y_{0}^{\prime}(x)$ ammetta un limite superiore finito, che rappresenteremo con $M$.

Per la continuità della funzione $f_{y^{\prime} y^{\prime}}$, possiamo determinare un numero positivo $\delta$ in modo che, soddisfatte le disuguaglianze

$$
|x-\bar{x}| \leq 2 \delta, \quad\left|y-y_{0}(\bar{x})\right| \leq 2 \delta, \quad\left|y^{\prime}-y_{0}^{\prime}(\bar{x})\right| \leq 2 \delta,
$$

ed essendo $(x y)$ un punto di $A$, sia

$$
f_{y^{\prime} y^{\prime}}\left(x, y, y^{\prime}\right)<-n,
$$

e ciò qualunque sia il punto $\bar{x}$ di $E$.

Scelto poi ad arbitrio un altro numero positivo $\varepsilon$, possiamo determinare un $\sigma$ maggiore di zero e minore di $\frac{1}{2} \delta$, cosi che:

$\left.I^{\circ}\right)$ se $x$ è un qualsiasi punto dell'intervallo $(a, b)$ su cui è definita la $y_{0}(x) \mathrm{e}$ $(x, y)$ è un punto di $A$ e sono soddisfatte le disuguaglianze $\left|y-y_{0}(x)\right|<\sigma,\left|y^{\prime}-\bar{y}^{\prime}\right|<\sigma$, $\left|y^{\prime}\right| \leq M+\delta$, sia

$$
\left|f\left(x, y_{0}(x), \bar{y}^{\prime}\right)-f\left(x, y, y^{\prime}\right)\right|<\varepsilon ;
$$

$\left.2^{\circ}\right)$ sia anche, su ogni insieme $a$ di $(a, b)$ di misura $m(a) \leq \sigma$,

$$
\left|\int_{a} f\left(x, y_{0}(x), y_{o}^{\prime}(x)\right) d x\right|<\varepsilon
$$

$\left.3^{\circ}\right)$ sia $\sigma . N<\varepsilon$, dove $N$ indica il massimo modulo della funzione $f$ nel campo

$$
a \leq x \leq b, \quad\left|y-y_{0}(x)\right| \leq \delta, \quad\left|y^{\prime}\right| \leq M+\delta ;
$$

$\left.4^{\circ}\right)$ La funzione $y_{0}(x)$ abbia, in ogni intervallo di ampiezza non superiore a $\sigma$, un'oscillazione $\leqslant \frac{1}{2} \delta$.

Ciò premesso, dividiamo il segmento $(a, b)$ in $n$ parti uguali e congiungiamo fra loro con segmenti rettilinei $\mathrm{i}$ consecutivi punti di $C_{0}$ che hanno per ascisse i punti di 
divisione ottenuti. Avremo cosi una poligonale $\Pi_{n}$, inscritta in $C_{o}$, la cui equazione potrà scriversi $y=y_{n}(x)$. Per $n \nrightarrow \infty$ la $y_{n}(x)$ tende uniformemente alla $y_{0}(x)$ su tutto $(a, b)$ e la lunghezza della poligonale $\Pi_{n}$ tende alla lunghezza della curva $C_{0}$. Possiamo dunque ${ }^{4 \mathrm{I}}$ ) determinare in intero $\bar{n}$ in modo che, per ogni $n>\bar{n}$ :

a) sia, per tutti gli $x$ di $(a, b)$,

$$
\left|y_{n}(x)-y_{0}^{\prime}(x)\right|<\sigma ;
$$

b) si abbia, in tutto $(a, b)$ ad eccezione al più di un insieme di misura minore di $\sigma$,

$$
\left|y_{n}^{\prime}(x)-y_{0}^{\prime}(x)\right|<\sigma ;
$$

c) l'insieme $E_{n}$ dei punti di $E$ in cui è

$$
\left|y_{n}^{\prime}(x)-y_{0}^{\prime}(x)\right|<\sigma
$$

risulti di misura maggiore di $\mu$.

Sia $n>\bar{n}$ e $E_{n}^{\prime}$ un insieme chiuso qualsiasi contenuto in $E_{n}$ e di misura maggiore di $\mu$.

Se $\bar{x}$ è un punto qualunque di $E_{n}^{\prime}$, possiamo determinare il massimo intervallo di $(a, b)$ contenente $\bar{x}$, avente gli estremi distanti da questo punto al più $\frac{\sigma}{2}$ e tutto contenuto nella proiezione sull'asse $x$ del lato di $\mathrm{I}_{n}$ a cui appartiene il punto $\left(x, y_{n}(\bar{x})\right)$. Fra gli intervalli cosi determinati possiamo sceglierne un numero finito che ricoprano tùtto l'insieme $E_{n}^{\prime}$; e ridotti gli intervalli di questo gruppo in modo che essi non si ricoprano scambievolmente, avremo un sistema di intervalli $l_{n, 1}, l_{n, 2}, \ldots, l_{n, m_{n}}$, non sovrapponentesi, ciascuno di ampiezza $\leqslant \sigma<\delta$, ricoprenti interamente $E_{n}^{\prime}$. Inoltre, per quanto precede, detto $x_{n, r}$ il primo estremo di $l_{n, r}$, è

$$
f_{y^{\prime} y^{\prime}}\left(x, y, y^{\prime}\right)<-n,
$$

per tutte le terne $\left(x, y, y^{\prime}\right)$ soddisfacenti alle disuguaglianze

$$
\left.\left|x-x_{n, r}\right| \leq \delta, \quad\left|y-y_{n}\left(x_{n, r}\right)\right| \leq \delta, \quad\left|y^{\prime}-y_{n}^{\prime}\left(x_{n, r}\right)\right| \leq \delta \quad{ }^{42}\right) .
$$

Ora, conformemente a quanto si è dimostrato al $\mathrm{n}^{0} 29$ possiamo asserire che, comunque piccolo si fissi un intorno delle parti dei lati di $\Pi_{n}$ corrispondenti ai segmenti

$4^{\text {I) }}$ loc. cit. ${ }^{35}$ ).

$\left.4^{2}\right)$ Per essere $l_{n, r}<\sigma<\delta$, è, se indichiamo con $\bar{x}$ il punto di $E_{n}^{\prime}$ dal cui intervallo è derivato $l_{n, r},\left|x_{n, r}-\bar{x}\right|<\sigma<\delta$ e, per $\left.a\right)$ e $\left.4^{\circ}\right),\left|y_{n}\left(x_{n, r}\right)-y_{0}\left(x_{n, r}\right)\right|+\left|y_{0}\left(x_{n, r}\right)-y_{0}(\bar{x})\right|<\sigma+\frac{1}{2} \delta<\delta$, ed ancora, essendo $y_{n}^{\prime}\left(x_{n, r}\right)=y_{n}^{\prime}(\bar{x}),\left|y_{n}^{\prime}\left(x_{n, r}\right)-y_{0}^{\prime}(\bar{x})\right|=\left|y_{n}^{\prime} \overline{(x)}-y_{0}^{\prime}(\bar{x})\right|<\sigma<\delta$, per $\left.c\right)$. 
$l_{n, r}$, è sempre possibile costruire per ciascuna di esse una poligonale $p_{n, r}$, aventi gli stessi estremi della parte considerata, appartenente all'intorno fissato e soddisfacente alla disuguaglianza

$$
\int_{x_{n, r}}^{x_{n, r}^{\prime}} f\left(x, y_{n, r}, y_{n, r}^{\prime}\right) d x-\int_{x_{n, r}}^{x_{n, r}^{\prime}} f\left(x, y_{n}, y_{n}^{\prime}\right) d x<-\frac{\mathrm{I}}{4} \eta l_{n, r} \delta^{2},
$$

dove $x_{n, r}^{\prime}$ indica il secondo estremo di $l_{n, r}$ e $y=y_{n, r}(x)$ è l'equazione della poligonale $p_{n, r}$, con $y_{n, r}^{\prime}(x)=y_{n}^{\prime}(x) \pm \delta$.

Consideriamo allora la curva $C_{n}$ formata con tutte le poligonali $p_{n, r}\left(r=\mathrm{I}, 2, \ldots, m_{n}\right)$, con le parti dei lati di $\Pi_{n}$ necessarie per collegare le $p_{n, r}$ alla curva $C_{0}$, e delle parti di quest'ultima curva indispensabili per il completamento della $C_{n}$ su tutto l'intervallo $(a, b)$. Indicata con $y=\varphi_{n}(x)$ l'equazione della $C_{n}$ e detti $\omega_{n, \mathrm{I}}, \omega_{n, 2}, \ldots, \omega_{n, m_{n}^{\prime}}$, gli intervalli di $(a, b)$ sui quali è $\varphi_{n}(x)=y_{n}(x)$, abbiamo

$$
\begin{aligned}
& \int_{0}^{b} f\left(x, \varphi_{n}(x), \varphi_{n}^{\prime}(x)\right) d x-\int_{a}^{b} f\left(x, y_{0}(x), y_{0}^{\prime}(x)\right) d x \\
& =\sum_{r=1}^{m_{n}}\left\{\int_{l_{n, r}} f\left(x, y_{n, r}, y_{n, r}^{\prime}\right) d x-\int_{l_{n, r}} f\left(x, y_{0}, y_{0}^{\prime}\right) d x\right\} \\
& +\sum_{r=1}^{m_{n}^{\prime}}\left\{\int_{\omega_{n r}} f\left(x, y_{n}, y_{n}^{\prime}\right) d x-\int_{\omega_{n r}} f\left(x, y_{0}, y_{0}^{\prime}\right) d x\right\} .
\end{aligned}
$$

$\mathrm{Ma} \dot{\mathrm{e}}$

$$
\begin{aligned}
& \int_{l_{n, r}} f\left(x, y_{n, r}, y_{n, r}^{\prime}\right) d x-\int_{l_{n, r}} f\left(x, y_{0}, y_{0}^{\prime}\right) d x \\
= & \left\{\int_{l_{n, r}} f\left(x, y_{n, r}, y_{n, r}^{\prime}\right) d x-\int_{l_{n, r}} f\left(x, y_{n}, y_{n}^{\prime}\right) d x\right\} \\
+ & \left\{\int_{l_{n, r}} f\left(x, y_{n}, y_{n}^{\prime}\right) d x-\int_{l_{n, r}} f\left(x, y_{0}, y_{o}^{\prime}\right) d x\right\}
\end{aligned}
$$

e per la $(56)$,

$$
<-\frac{\mathrm{I}}{4} \eta l_{n, r} \delta^{2}+\left\{\int_{l_{n, r}} f\left(x, y_{n}, y_{n}^{\prime}\right) d x-\int_{l_{n, r}} f\left(x, y_{0}, y_{0}^{\prime}\right) d x\right\} .
$$

La somma delle lunghezze dei segmenti $l_{n, r}\left(r=1,2, \ldots, m_{n}\right)$ non potendo risultare minore della misura dell'insieme $E_{n}^{\prime}$, completamente ricoperto dagli $l_{n, r}$, misura che è maggiore di $\mu$, abbiamo

$$
\left\{\begin{array}{c}
\int_{a}^{b} f\left(x, \varphi_{n}, \varphi_{n}^{\prime}\right) d x-\int_{a}^{b} f\left(x, y_{0}, y_{0}^{\prime}\right) d x<-\frac{\mathrm{I}}{4} n \mu \delta^{2} \\
+\sum_{r=1}^{m_{n}}\left\{\int_{q_{n, r}} f\left(x, y_{n}, y_{n}^{\prime}\right) d x-\int_{q_{n, r}} f\left(x, y_{0}, y_{0}^{\prime}\right) d x\right\},
\end{array}\right.
$$


dove con $q_{n, r}$ si indicano quelle proiczioni su $(a, b)$ dei lati di $\Pi_{n}$ le quali contengono i segmenti $l_{n, r}$ e gli $\omega_{u, r}$.

Osserviamo che, per la scelta fatta dellindice $n$ [condiz. $a$ ) e $b$ )] e per $1 a 1^{\circ}$ ), su tutti gli intervalli $q_{n r}$ ad eccezione, al più di un insieme di punti di misura $<\sigma$, è

$$
\left|f\left(x, y_{n}, y_{n}^{\prime}\right)-f\left(x, y_{0}, y_{o}^{\prime}\right)\right|<\varepsilon,
$$

perchè ogni $q_{n, r}$ contiene almeno un punto di $E_{n}^{\prime}$, e in tal punto è $\left|y_{n}^{\prime}-y_{0}^{\prime}\right|<\sigma$ [cond. c)] e perciò $\left|y_{n}^{\prime}\right|<M+\sigma<M+\delta$, la quale disuguaglianza risulta verificata su tutto $q_{n, r}$, essendo, su ciascuno di questi seguenti, $y_{n}^{\prime}$ costante. Se dunque teniamo conto della $(58)$ e delle condizioni $\left.a), 2^{\circ}\right), 3^{\circ}$ ), abbiamo

$\left|\sum_{r=1}^{m_{n}}\left\{\int_{q_{n, r}} f\left(x, y_{n}, y_{n}^{\prime}\right) d x-\int_{q_{n, r}} f\left(x, y_{0}, y_{0}^{\prime}\right) d x\right\}\right|<\varepsilon(b-a)+\varepsilon+\varepsilon=\varepsilon(b-a+2)$, e per la (57)

$$
\int_{a}^{b} f\left(x, y_{n}, y_{n}^{\prime}\right) d x-\int_{a}^{b} f\left(x, y_{0}, y_{0}^{\prime}\right) d x<-\frac{1}{4} n \mu \delta^{2}+\varepsilon(b-a+2) .
$$

Siccome $\varepsilon \dot{e}$ in nostro arbitrio, possiamo prenderlo in modo che sia

ne viene allora

$$
\varepsilon<\frac{n \mu \delta^{2}}{8(b-a+2)}
$$

$$
\int_{a}^{b} f\left(x, y_{n}, y_{n}^{\prime}\right) d x-\int_{a}^{b} f\left(x, y_{0}, y_{0}^{\prime}\right) d x<-\frac{1}{8} \eta \mu \delta^{2} .
$$

La curva $C_{n}$ potendo prendersi in modo da appartenere ad un intorno prefissato a piacere della $C_{o}$, la nostra proposizione è completamente dimostrata.

Osservazione. - Si puó qui ripetere integralmente l'Osservazione II posta in fine al $\mathrm{n}^{\circ}$ precedente.

57. Ripetendo il ragionamento fatto or ora, con l'avvertenza di sostituire alla disuguaglianza (56) l'altra:

$$
\int_{x_{n, r}}^{x_{n, r}^{\prime}} f\left(x, y_{n, r}, y_{n, r}^{\prime}\right) d x-\int_{x_{n, r}}^{x_{n, r}^{\prime}} f\left(x, y_{n}, y_{n}^{\prime}\right) d x<-\frac{\mathrm{I}}{4} r_{i} l_{n, r} \lambda,
$$

che si ottiene procedendo come al $\mathrm{n}^{0} 34$, si giunge alle proposizioni :

a) Condizione necessaria affinche su una data curva $C_{\mathrm{o}}\left(\mathrm{n}^{\circ} 40\right)$, completamente interna al campo $A$ e per la quale esista ovunque e sempre sia continua la derivata $y_{0}^{\prime}(x)$, lintegrale $I_{C}$ sia funzione semicontinua inferiormente, \& che si abbia in ogni punto di $C_{\text {o }}$

$$
E\left(x, y_{0}(x) ; y_{0}^{\prime}(x), y^{\prime}\right) \geqslant 0,
$$

per tutti i possibili valori di $y^{\prime}$. 
b) Condizione necessaria affincbè su una data curva $C_{\mathrm{o}}$ (n. 40), completamente interna al campo $A$, l'integrale $I_{C}$ sia funzione semicontinua inferiormente, $\dot{e}$ che abbia misura nulla linsieme dei valori di $x$ nei quali non è verificata, per tutti $i$ possibili valori di $y^{\prime}$, la disuguaglianza

$$
E\left(x, y_{0}(x) ; y_{0}^{\prime}(x), y^{\prime}\right) \gg \mathrm{o} .
$$

Osservazione. - Vale anche per queste due proposizioni quanto si è detto nell'Osservazione II del $\mathbf{n}^{\circ} 55$.

Parma, agosto I9Ig.

LEONIDA TONELLI. 